Florida International University FIU Digital Commons

$10-17-2017$

\title{
Detecting Structural Defects Using Novel Smart Sensory and Sensor-less Approaches
}

Amin Baghalian

Florida International University, abagh004@fiu.edu

DOI: 10.25148 /etd.FIDC004006

Follow this and additional works at: https://digitalcommons.fiu.edu/etd

Part of the Acoustics, Dynamics, and Controls Commons, Electro-Mechanical Systems

Commons, and the Signal Processing Commons

\section{Recommended Citation}

Baghalian, Amin, "Detecting Structural Defects Using Novel Smart Sensory and Sensor-less Approaches" (2017). FIU Electronic Theses and Dissertations. 3560.

https://digitalcommons.fiu.edu/etd/3560

This work is brought to you for free and open access by the University Graduate School at FIU Digital Commons. It has been accepted for inclusion in FIU Electronic Theses and Dissertations by an authorized administrator of FIU Digital Commons. For more information, please contact dcc@fiu.edu. 


\title{
FLORIDA INTERNATIONAL UNIVERSITY
}

\author{
Miami, Florida
}

\section{DETECTING STRUCTURAL DEFECTS USING NOVEL SMART SENSORY AND SENSOR-LESS APPROACHES}

A dissertation submitted in partial fulfillment of

the requirements for the degree of

DOCTOR OF PHILOSOPHY

in

MECHANICAL ENGINEERING

by

Amin Baghalian 
To: Dean John L. Volakis

College of Engineering and Computing

This dissertation, written by Amin Baghalian, and entitled Detecting Structural Defects Using Novel Smart Sensory and Sensor-less Approaches, having been approved in respect to style and intellectual content, is referred to you for judgment.

We have read this dissertation and recommend that it be approved.

$\begin{array}{r}\text { Cesar Levy } \\ \hline \text { Dwayne McDaniel } \\ \hline \text { Yiding Cao } \\ \hline \text { Igor Tsukanov } \\ \hline \text { Ibrahim Nur Tansel, Major Professor }\end{array}$

Date of Defense: Oct 17, 2017

The dissertation of Amin Baghalian is approved.

\section{Dean John L. Volakis College of Engineering and Computing}

Andrés G. Gil Vice President for Research and Economic Development and Dean of the University Graduate School

Florida International University, 2017 
(C) Copyright 2017 by Amin Baghalian

All rights reserved. 


\section{DEDICATION}

I would like to dedicate this dissertation to my beloved parents Rana and Behnam, and my dear sister Alaleh and at last but not the least to my lovely wife Nasim which without her support and endless love, the completion of the work would not have been possible. I also would like to dedicate this work to all those incredible people who helped me to improve personally and professionally. 


\section{ACKNOWLEDGMENTS}

I am greatly thankful to god and many people who helped me throughout my $\mathrm{PhD}$ studies. I am deeply indebted to my advisor, Dr. Ibrahim N. Tansel for his guidance and encouragement throughout all phases of my study. I sincerely thank members of my dissertation committee Dr. Cesar Levy, Dr. Dwayne McDaniel, Dr. Yiding Cao, and Dr. Igor Tsukanov for their invaluable suggestions, comments, and support throughout all stages of my academic work. I am very fortunate and grateful to have you as my advisors and lifelong mentors.

As a member of the FIU Mechatronics Lab, I had the chance to work with many intelligent researchers, scholars, and sincere friends. I am thankful to all students who worked with me especially Mr. Jason Soto and Mr. Ali Qureshi. My special thank goes to my lab mates Mr. Shervin Tashakori, Dr. Volkan Senyurek, and all other research team members for making our Lab an excellent scientific environment and an enjoyable workplace.

I sincerely express my gratitude to the staff of the Mechanical Engineering Department especially Mariam Barrueco and Mabel Fernandez for their help and support.

I would like to acknowledge the University Graduate School, Florida International University for providing support for this research in the form of Doctoral Evidence Acquisition (DEA) and Dissertation Year Fellowships (DYF). I would like to thank the Graduate \& Professional Student Committee (GPSC) for the support received through conference travel grants to present my research at conferences.

I am extremely thankful to my lovely wife, family, and friends. None of these would have been possible without their endless love, support, inspiration, and prayers. 


\section{ABSTRACT OF THE DISSERTATION \\ DETECTING STRUCTURAL DEFECTS USING NOVEL SMART SENSORY AND SENSOR-LESS APPROACHES}

by

Amin Baghalian

Florida International University, 2017

Miami, Florida

Professor Ibrahim Nur Tansel, Major Professor

Monitoring the mechanical integrity of critical structures is extremely important, as mechanical defects can potentially have adverse impacts on their safe operability throughout their service life. Structural defects can be detected by using active structural health monitoring (SHM) approaches, in which a given structure is excited with harmonic mechanical waves generated by actuators. The response of the structure is then collected using sensor(s) and is analyzed for possible defects, with various active SHM approaches available for analyzing the response of a structure to single- or multi-frequency harmonic excitations.

In order to identify the appropriate excitation frequency, however, the majority of such methods require a priori knowledge of the characteristics of the defects under consideration. This makes the whole enterprise of detecting structural defects logically circular, as there is usually limited a priori information about the characteristics and the locations of defects that are yet to be detected. Furthermore, the majority of SHM 
techniques rely on sensors for response collection, with the very same sensors also prone to structural damage.

The Surface Response to Excitation (SuRE) method is a broad band frequency method that has high sensitivity to different types of defects, but it requires a baseline. In this study, initially, theoretical justification was provided for validity of the SuRE method and it was implemented for detection of internal and external defects in pipes. Then, the Comprehensive Heterodyne Effect Based Inspection (CHEBI) method was developed based on the SuRE method to eliminate the need for any baseline.

Unlike traditional approaches, the CHEBI method requires no a priori knowledge of defect characteristics for the selection of the excitation frequency. In addition, the proposed heterodyne effect-based approach constitutes the very first sensor-less smart monitoring technique, in which the emergence of mechanical defect(s) triggers an audible alarm in the structure with the defect. Finally, a novel compact phased array (CPA) method was developed for locating defects using only three transducers. The CPA approach provides an image of most probable defected areas in the structure in three steps. The techniques developed in this study were used to detect and/or locate different types of mechanical damages in structures with various geometries. 


\section{TABLE OF CONTENTS}

CHAPTER

PAGE

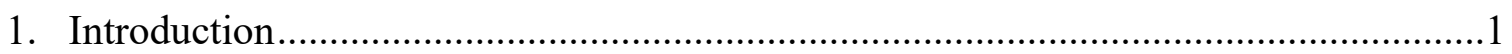

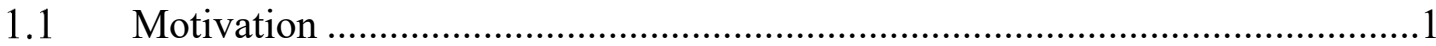

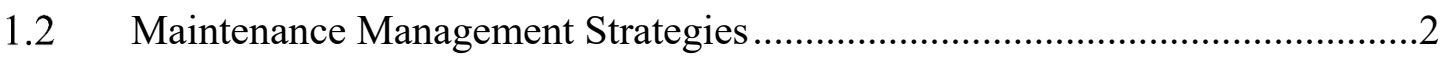

$1.3 \quad$ Ultrasonic Detection Strategies …………….............................................

1.4 Active and Passive Approaches in SHM .......................................................

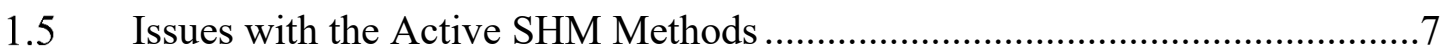

1.6 Research Goals, Scope, and Objectives..........................................................11

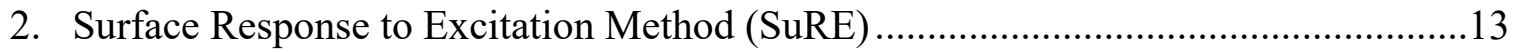

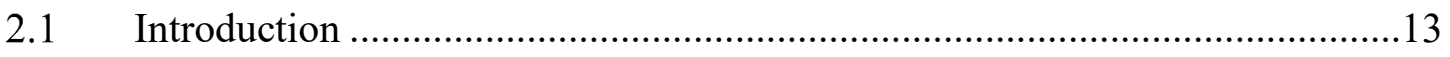

2.2 Theoretical Background \& Proposed Method ...............................................17

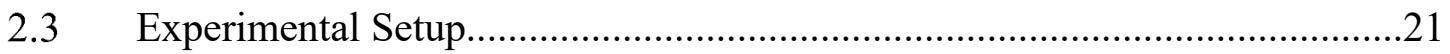

2.3.1 Detection of defects using bonded piezoelectric transducers ......................21

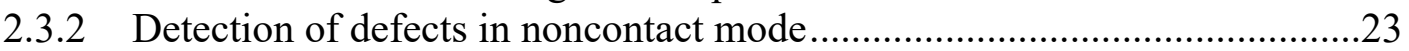

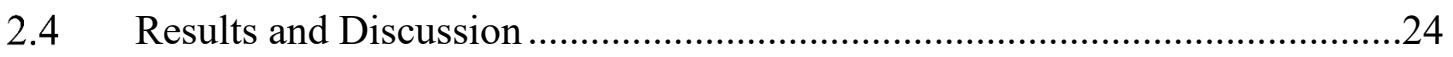

2.4.1 Detection of defects using bonded piezoelectric transducers ......................24

2.4.2 Detection of defects in noncontact mode....................................................

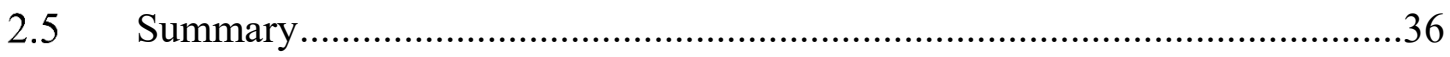

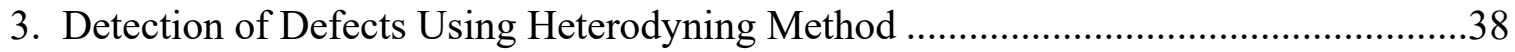

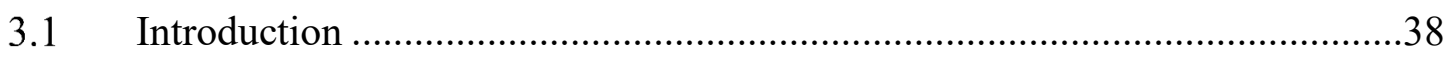

3.2 Theoretical Background and Numerical Model ...........................................41

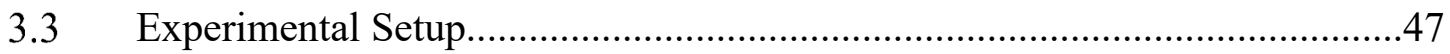

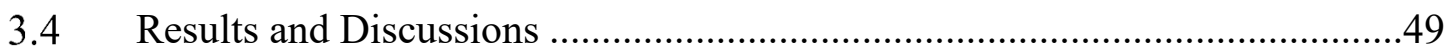

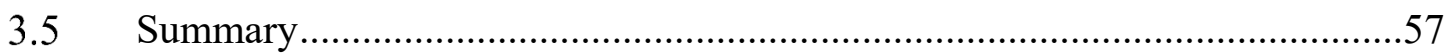

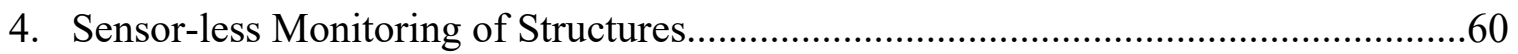

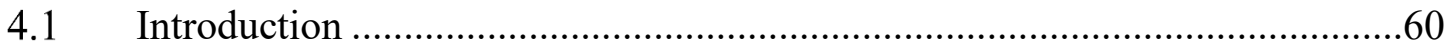

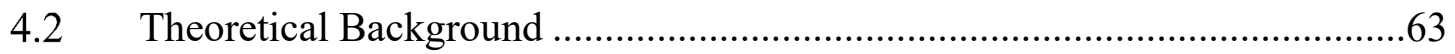

$4.3 \quad$ Experimental Setup.................................................................................64

4.3.1 Test with two plates held together with four bolts:......................................64

4.3.2 Implementation of the SSHM in industrial washers .....................................65

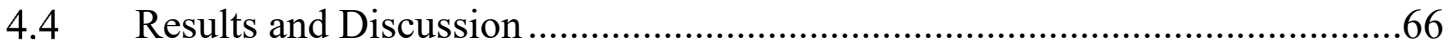

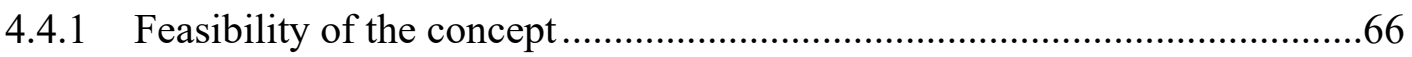

4.4.2 Quantification of bolt tightness using the SSHM method ............................69

$4.5 \quad$ Summary ...............................................................................................

5. Localization of Multiple Defects Using the Compact Phased Array (CPA) Method ..79 


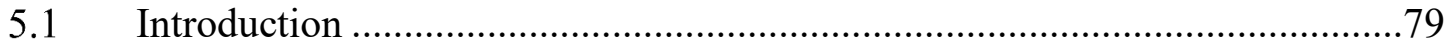

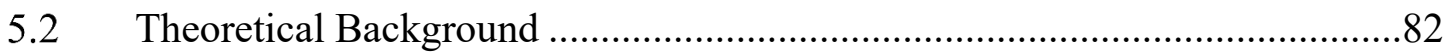

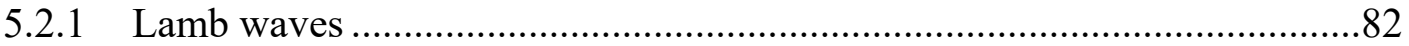

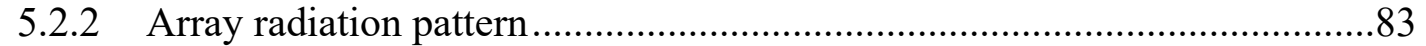

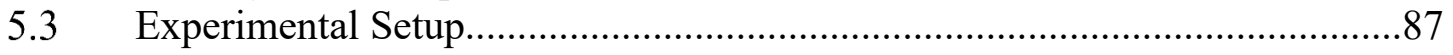

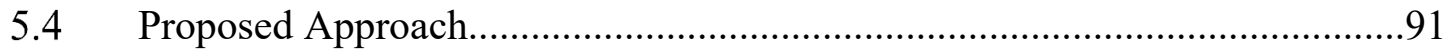

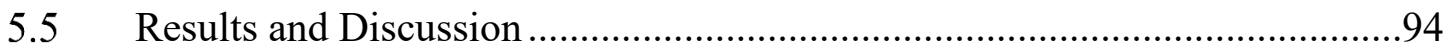

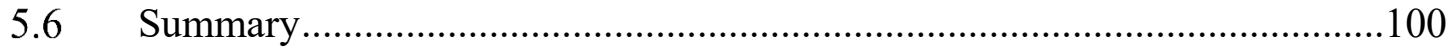

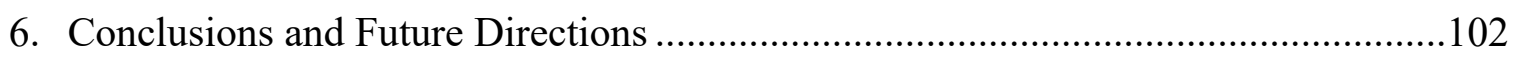

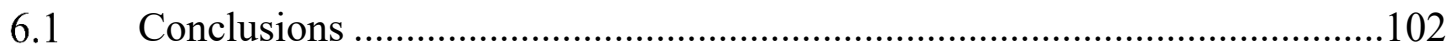

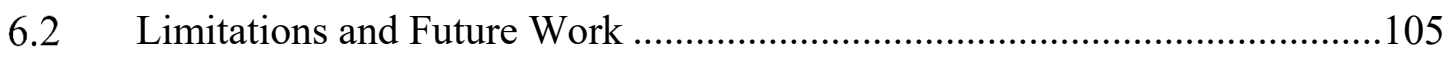

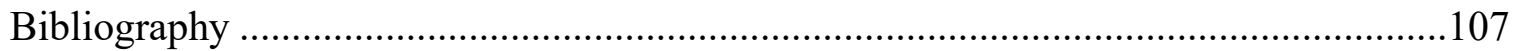

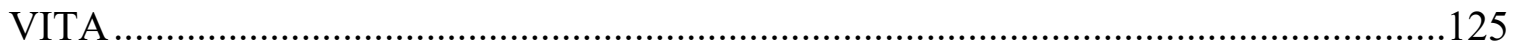




\section{LIST OF TABLES}

TABLE

PAGE

Table 1.1 Comparison between different NDT\&E methods.

Table 3.1 Amplitudes of 5 frequency components on $\mathrm{f}_{2}-\mathrm{f}_{1}$ line.

Table 5.1 Different combinations in the experimental procedure .88

Table 5.2 List of damages and their characteristics.

Table 5.3 Estimated location of multiple defects and associated errors in angular and radial directions. 100 


\section{LIST OF FIGURES}

FIGURE

PAGE

Figure 1.1 Distortion of the signals due to dispersion in different distances from the actuator.

Figure 1.2 Dispersion curve for an aluminum plate.

Figure 1.3 Dispersion curve for an aluminum pipe.

Figure 2.1 Experimental setup for implementation of the SuRE method in contact mode.

Figure 2.2 Experimental setup for implementation of the SuRE method in non-contact mode.

Figure 2.3 External longitudinal damages with the same depth of $0.75 \mathrm{~mm}$ and length of a) $22.93 \mathrm{~mm} \mathrm{b)} 45.86 \mathrm{~mm} \mathrm{c)} 68.79 \mathrm{~mm}$.

Figure 2.4 Comparison between initial baseline response and responses obtained at sensor location one after different damage lengths.

Figure 2.5 SSD damage index values before and after the creation of the external longitudinal damages at all sensor locations.

Figure 2.6 Damage index values at four sensor locations before and after the increases in the depth of the external longitudinal damage with constant length of $68.79 \mathrm{~mm} \ldots \ldots . .28$

Figure 2.7 Internal longitudinal damages with the fixed depth of $0.75 \mathrm{~mm}$.

Figure 2.8 SSD damage index values at four sensor locations before and after the increases in the length of the internal longitudinal damage.

Figure 2.9 Damage index values at four sensor locations before and after the increases in depth of the $68.7 \mathrm{~mm}$ length longitudinal damage. 
Figure 2.10 1st Baseline spectrum vs. 2nd Baseline spectrum.

Figure 2.11 Frequency responses of the pipe after different levels of the circumferential damage at sensor location $1 . .$.

Figure 2.12 SSD damage index values at eight sensory points before and after the increases in depth of the circumferential damage.

Figure 2.13 SSD damage index values at eight sensor locations before and after the increases in the length of the longitudinal damage.

Figure 2.14 SSD damage index values at eight sensor locations before and after the increases in depth of the $100 \mathrm{~mm}$ length longitudinal damage. .36

Figure 3.1 Schematic of applied excitations using two distinctive frequencies.

Figure 3.2 Fast Fourier Transform (FFT) of the response signal for the structure with nonlinearity.

Figure 3.3 The Short Time Fourier Transform (STFT) of the response signals for the pristine and with crack specimens.

Figure 3.4 Schematic of experimental setup.

Figure 3.5 Pictures of the crack at the beginning (a) and end (b).

Figure 3.6 The STFT of the response of the structure with $1 \mathrm{~mm}$ long crack to the double sweep excitation.

Figure 3.7 The STFT of the response of the pristine specimen to the double sweep excitation.

Figure 3.8 The STFT of the response of the structure with $25 \mathrm{~mm}$ long crack to the double sweep excitation. 
Figure 3.9 The STFT of the response of the structure with $19 \mathrm{~mm}$ long crack to the double sweep excitation.

Figure 3.10 The STFT of the response of the structure with $13 \mathrm{~mm}$ long crack to the double sweep excitation.

Figure 3.11 The STFT of the response of the structure with $7 \mathrm{~mm}$ long crack to the double sweep excitation.

Figure 3.12 The STFT of the response of the structure with $3 \mathrm{~mm}$ long crack to the double sweep excitation.

Figure 3.13 Tests for detection of loose bolt using a combination of broad band frequency and single frequency a) plates system b) washer system.

Figure 3.14 Response of the plate to double excitation a) loose bolt b) tightened by $2 \mathrm{Nm}$.

Figure 3.15 Response of the washer system to double excitation a) loose bolt b) tightened by $2 \mathrm{Nm}$.

Figure 4.1 SSHM for detection of the loose bolt in the plates structure

Figure 4.2 Schematic of the SSHM system for the washer system. .66

Figure 4.3 Recorded response of the plate system to the excitation

Figure 4.4 Recorded response of the washer system to the excitation. .68

Figure 4.5 The FFT of the response of the washer system to the sweep excitation in the range of $50-250 \mathrm{kHz}$.

Figure 4.6 The audible sweep excitation in the range of $100 \mathrm{~Hz}-20 \mathrm{kHz}$. .70

Figure 4.7 The FFT of the applied modulated signal .71 
Figure 4.8 The recorded audible response.

Figure 4.9 The FFT of the recorded response. .72

Figure 4.10 The comparison between audible response of the washer system in different torques. .73

Figure 4.11 The comparison between the FTT values of the audible responses of the washer system in different torques.

Figure 4.12 The comparison between the RMS values of the audible responses of the washer system at different torques.

Figure 4.13 The comparison between the RMS values of the audible responses of the washer installed in the plate system in different torques.

Figure 4.14 The comparison between the FFT of the audible responses of the washer installed in the plate system in different torques.

Figure 4.15 The comparison between the RMS values of the audible responses of the washer system installed in the plate system in different torques

Figure 5.1 Resultant radiation pattern of a two-element linear phased array system for different element spacing: a) $d=0.5 \lambda$ b) $d=0.75 \lambda$ c) $d=\lambda$.

Figure 5.2 a) Excitation signal (five cycle hamming windowed sine wave)

b) frequency spectrum and phase speed at A0 mode for the test plate (Al-2024).

Figure 5.3 Radiation pattern: red for five cycles hamming windowed sine tone and blue for a single tone.

Figure 5.4 Experiment setup for the compact phased array approach.

Figure 5.5 Propagation pattern for different exciter combinations, green points show the actuators and the white ones show the receiver. a) Combination 1 b) Combination 2 c) Combination 3 
Figure 5.6 The test plate, PZTs, and damages.

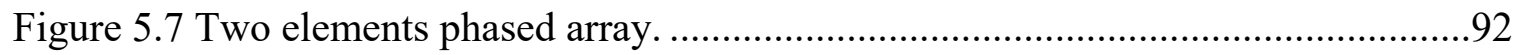

Figure 5.8 Simplified flow chart of the proposed method..........................................94

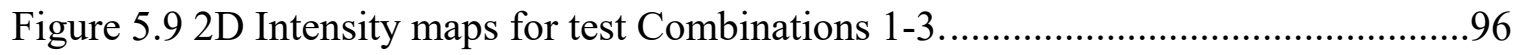

Figure 5.10 Total normalized intensity maps a) Intensity map resulted from the modified approach b) Intensity map of (a) after thresholding of \%3. c) Intensity map resulted from standard approach d) Intensity map of (c) after thresholding of \%3........97

Figure 5.11 Estimated damage location (Black area shows real location of the damage).98

Figure 5.12 a) total normalized intensity map b) final estimated damage locations and actual damage locations. . 


\section{CHAPTER 1}

\section{Introduction}

\subsection{Motivation}

The United States infrastructures are facing aging problem and there are meaningful gaps between excellent operating conditions and the current state [1]-[5]. Aircraft, railroads, bridges, and pipelines are some examples of critical infrastructures, and ensuring their safety is of paramount importance for the structural health monitoring (SHM) community. About 60 percent of failures in aircraft are due to fatigue cracks and by using conventional methods, they are usually detected when they reach about 80 percent of their fatigue life [6]. The U.S. has 614,387 bridges, and many are approaching the end of their design life. About $40 \%$ of them are 50 years or older and about one out of 10 bridges were identified as structurally deficient in 2016 [7]. About one-third of U.S. exports and transportation of 85 thousand passengers take place through railroads, which are going to face serious challenges due to aging issues. Based on the 2017 infrastructure report card, aviation, pipelines, bridges, and railroads are graded as $\mathrm{D}, \mathrm{D}+, \mathrm{C}+$, and $\mathrm{B}$, which shows the gap between excellent operating condition and the current situation [5].

Statistics provided by the Pipeline and Hazardous Materials Safety Administration (PHZMA) of the U.S. Department of Transportation (DOT) [8] reveals that in America, there were 11,496 incidences in oil, gas, and hazardous liquid pipelines between 1997 and 2016, which resulted in 324 fatalities and 1331 injuries and a total cost of $\$ 7,050,142,502$. The share of failures due to corrosion, cracking, loose connections, and other undetected mechanical defects due to excavations and welding defects were about 2659 incidences. 
The cost of these incidences was $\$ 2,258,754,922$, which accounts for $32.04 \%$ of total failure incident costs. By considering the performance of current inspection methods, development of more accurate and cost-effective SHM techniques that can be conveniently applied for online monitoring of structures seems to be a must for proper maintaining of the safety of aging structures.

\subsection{Maintenance Management Strategies}

Reactive, preventive, and predictive maintenance are the three main strategies in the maintenance of aging infrastructure [9]. The reactive strategy refers to repair after failure and is the costliest approach. Therefore, reactive strategies have been used in less critical applications [10]. Preventive strategies consist of inspection/maintenance activities that are operated based on pre scheduled timelines. Cost of preventive maintenance management is about one-third of the reactive approaches [11]. SHM approaches fall under the predictive approach which is also known as condition based monitoring approach. Here, the focus is on maximizing the in-service life of structures and only making the repair or replacement when necessary [12], [13].

\subsection{Ultrasonic Detection Strategies}

A damage can be defined as a change in a structural system which adversely affects the mechanical integrity of the structure. Typically, no sensor is capable of measuring damages independently, but they convert a physical medium, such as temperature, strain, light, sound, and vibration into processable signals [14]. By extracting the effects of a damage on the measured physical quantity and through the implementation of appropriate identification algorithms, the damage can be identified. There are two main strategies 
available for defect detection: Nondestructive testing and evaluation (NDT\&E) [15] and structural health monitoring (SHM) [16]. A brief comparison between different NDT\&E methods [15], [24] is shown in Table 1 and as can be seen each method has its advantages and disadvantages.

Table 1.1 Comparison between different NDT\&E methods.

\begin{tabular}{|c|c|c|c|c|c|}
\hline Event & $\begin{array}{c}\text { Penetrant } \\
\text { Testing }\end{array}$ & $\begin{array}{l}\text { Magnetic } \\
\text { Particle } \\
\text { Testing } \\
\end{array}$ & $\begin{array}{c}\text { Eddy } \\
\text { Current } \\
\text { Testing } \\
\end{array}$ & $\begin{array}{c}\text { Radiographic } \\
\text { Testing }\end{array}$ & $\begin{array}{c}\text { Ultrasonic } \\
\text { Testing }\end{array}$ \\
\hline $\begin{array}{l}\text { Internal } \\
\text { Defects }\end{array}$ & $x$ & $\begin{array}{l}\text { Near surface } \\
\text { defects }\end{array}$ & $\begin{array}{c}\text { Near surface } \\
\text { defects }\end{array}$ & $\checkmark$ & $\checkmark$ \\
\hline $\begin{array}{c}\text { External } \\
\text { Defects }\end{array}$ & $\begin{array}{l}\text { Surface } \\
\text { breaking } \\
\text { defects }\end{array}$ & $\checkmark$ & $\checkmark$ & $\checkmark$ & $\checkmark$ \\
\hline $\begin{array}{c}\text { Minimum } \\
\text { Surface } \\
\text { Preparation }\end{array}$ & $\begin{array}{l}\text { Surface } \\
\text { preparation } \\
\text { is critical }\end{array}$ & $\begin{array}{c}\text { Requires } \\
\text { relatively } \\
\text { smooth surface }\end{array}$ & $\checkmark$ & $\checkmark$ & $\checkmark$ \\
\hline $\begin{array}{l}\text { Non-contact } \\
\text { Monitoring }\end{array}$ & $x$ & $x$ & $\checkmark$ & $\begin{array}{l}\text { Access to both } \\
\text { sides of the } \\
\text { structure is } \\
\text { usually } \\
\text { required }\end{array}$ & $\checkmark$ \\
\hline Low Hazard & $\begin{array}{l}\text { Chemical } \\
\text { handling } \\
\text { precautions } \\
\text { are } \\
\text { necessary }\end{array}$ & $\begin{array}{c}\text { Post cleaning is } \\
\text { usually } \\
\text { necessary }\end{array}$ & $\checkmark$ & $\begin{array}{l}\text { Possible } \\
\text { radiation } \\
\text { hazard for } \\
\text { personnel }\end{array}$ & $\checkmark$ \\
\hline $\begin{array}{l}\text { Applicability } \\
\text { to Different } \\
\text { Materials and } \\
\text { Structures }\end{array}$ & $\begin{array}{l}\text { Requires a } \\
\text { relatively } \\
\text { smooth and } \\
\text { nonporous } \\
\text { surface } \\
\end{array}$ & $\begin{array}{c}\text { Only } \\
\text { ferromagnetic } \\
\text { material }\end{array}$ & $\begin{array}{c}\text { Only } \\
\text { conductive } \\
\text { material }\end{array}$ & $\checkmark$ & $\checkmark$ \\
\hline $\begin{array}{l}\text { Low Skill and } \\
\text { Training }\end{array}$ & $\checkmark$ & $\checkmark$ & $x$ & $x$ & $x$ \\
\hline
\end{tabular}


A large variety of electromagnetic, thermal, ultrasonic sensors and technologies have been deployed for collection of data from structures. Dye penetrant testing [17], modal testing [18], [19], radiography [18], ultrasonic [20], infrared thermography [21], eddy current [22] and X-ray tomography [23] are some of various NDT\& E methods available for assessment of mechanical integrity of structures.

Ultrasonic methods are dynamic methods that are used for identification and quantification of damages through analyzing the dynamic behavior of a system. Usually, ultrasonic NDT\&E approaches require bulky and costly instruments that are not permanently bonded to the structures and are operated in scheduled intervals when the structure is not in service. Generally, due to the point by point measurements and the resulting local nature of inspection of these approaches, monitoring of large structures is very time consuming and costly. For example, the time that is required for grounding an aircraft for inspection consists of about $27 \%$ of their service life [25]. However, down time is not desirable in any industry and can cost hundreds of thousands of dollars per hour [26].

Implementation of permanent and continuous monitoring schemes for detection and identification of damages are referred to as structural health monitoring (SHM) [27]. SHM methods differ from NDT and NDE approaches in that integrated sensing devices are used for in-situ monitoring of structures. Therefore, SHM holds certain advantages over conventional NDT\&E approaches: First, the continuous monitoring helps to minimize the possibility of catastrophic failures in between scheduled inspections. Second, it reduces the downtime of the systems since they can be performed while the structure is in service. 
Third, it eliminates the need for assembling and dissembling of inspection devices in each examination.

\subsection{Active and Passive Approaches in SHM}

SHM methods can be put into two main groups of active and passive methods [28], [29]. Passive methods do not involve any actuator in the monitoring process and therefore no mechanical energy is intentionally imparted to the structure under the test. Acoustic Emissions (AE) [30] is the most well-known passive method. The rapid release of energy from events, such as cracks and corrosions in general and debondings, delaminations, and matrix breakings in composite materials manifest themselves in the form of acoustic signals mainly in the frequency range of $100 \mathrm{kHz}$ to $1 \mathrm{MHz}$. In spite of the simplicity of the process, interpretation of $\mathrm{AE}$ signals for detection and locating the source of $\mathrm{AE}$ signals is very complicated. Active methods excite structures using actuator(s) and sensor(s) collect the response of the structure to the known input [31].

Ultrasonic monitoring methods have emerged as promising tools to overcome the shortcoming of the available methods in the nondestructive monitoring of small damages and monitoring of the intrinsic area of objects. Ultrasound waves are elastic mechanical waves and therefore they can propagate in any media but not vacuum. Ultrasonic bulk waves are a group of ultrasonic waves that freely propagate inside the bulk of materials and don't need any boundaries for their propagation [32]. Bulk waves are generally produced by surface tapping. These waves have a constant velocity at a constant frequency and therefore are easy to use and easy to understand. However, they can only provide point by point measurements. Guided-waves are the primary type of waves that are used in SHM 
since they can propagate for long distances along the length of a structure while maintaining reasonable sensitivity [33]-[35].

Traditionally, in sound and ultrasound based methods, microphones, strain gauges, and accelerometers are used for monitoring dynamic behavior of structures in form of electroresistive, capacitive or inductive devices. Recently, these sensors have been replaced by small and inexpensive piezoelectric transducers [36]. Piezoelectric materials have been effectively used for excitation and sensing of guided waves. Piezoelectricity is a reversible phenomenon which was discovered by the Curie brothers in 1880 .

For the direct piezo electric effect, an electrical voltage is produced when mechanical stress is applied to the material. In indirect piezoelectric effect, mechanical stress is produced by applying an electrical voltage to the material. Some natural materials such as quartz ( $\mathrm{SiO} 2)$, Rochelle salt and tourmaline exhibit moderate levels of the piezo electric effect. Industrial piezoelectric materials are manufactured to provide transducers with controlled piezoelectric properties. Two widely manufactured piezoelectric transducers are lead zirconate titanate (PZT) and polyvinylidene fluoride (PVDF). PVDF transducers show better sensing properties compared to their actuation capabilities. However, PZT transducers show strong direct and indirect piezoelectricity effects and therefore they are widely used for both excitation and sensing. Due to the small size, flexibility in shape, and cheap price these transducers have expedited the transformation from scheduled based monitoring in NDT to in-situ monitoring in SHM. 


\subsection{Issues with the Active SHM Methods}

Guided waves are dispersive [37]-[39]. That means the group and phase velocity of propagating guided waves depend on the frequency and mode of excitation. Also, different modes can exist in each frequency. Therefore, even in single frequencies a wave can propagate with different speeds and thus a wave distorts during its propagation from the exciter to the sensor. For a better illustration of the concept, distortions of five cycles of a $100 \mathrm{kHz}$ tone burst input signal after four different propagation distances are calculated using waveform revealer software [40] and presented in Figure 1.1.

Dispersion makes the signal analysis and interpretation a troublesome and challenging task. Anti-symmetric and symmetric modes are the two main modes in plate like structures while flexural, torsional, and longitudinal modes are the primary modes in hollow cylindrical structures. Dispersion graphs for an aluminum plate and pipe are shown in Figure 1.2 and Figure 1.3, respectively.

The primary approach for overcoming the dispersion and resulting complicatedness in signal understanding, in the time domain, is to only excite certain modes while suppressing the others [41]-[43]. This approach can make the signal understanding easier. However, it must be noted that sensitivities of different modes to various potential damages are not the same. Also, in practice, there is no or limited a priori knowledge about the characteristics of damages that might be present in the structure. Therefore, in single excitation frequency approaches, the fitness of the selected excitation frequency to the damage that is yet to be detected plays an important role in successful defect detection. 

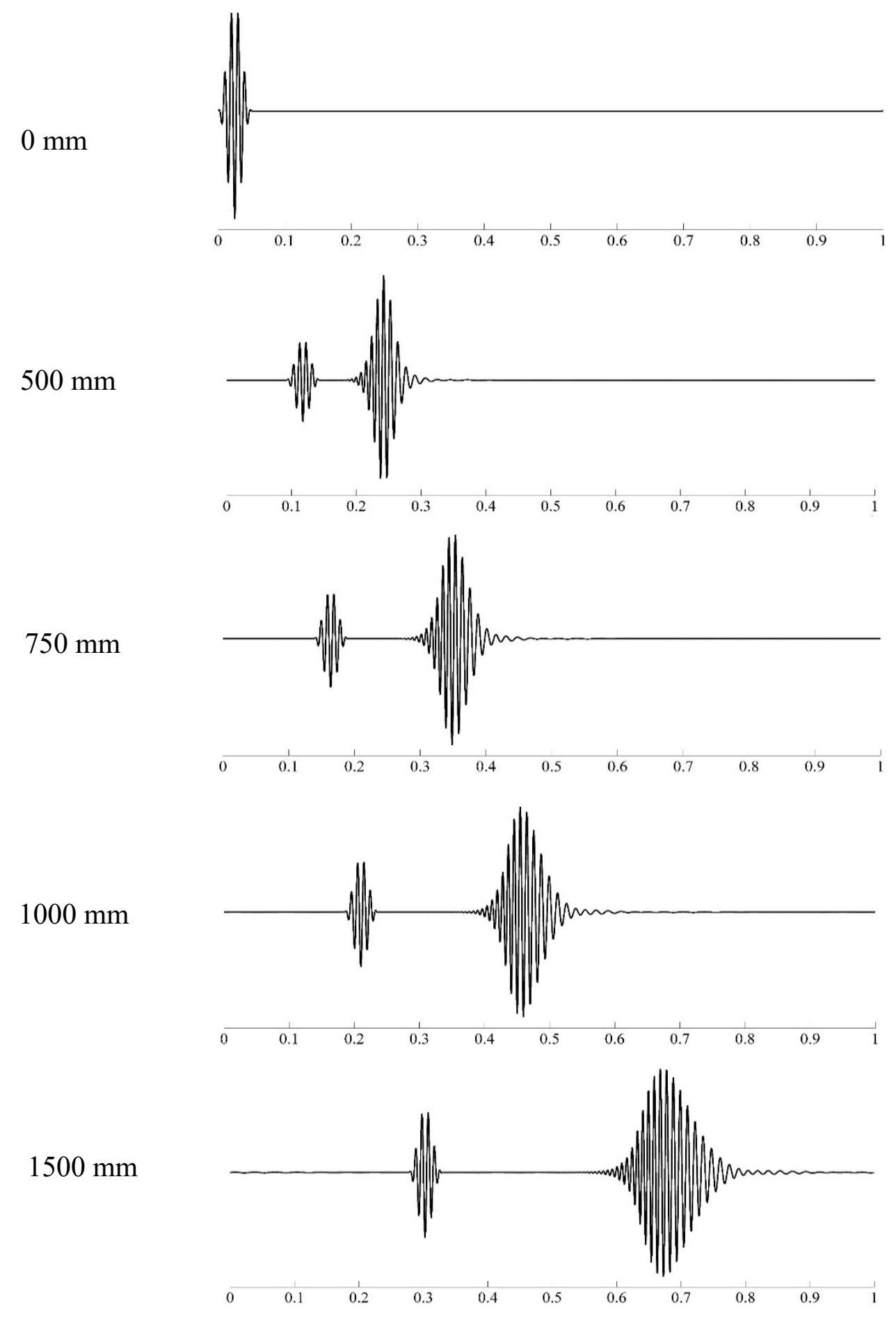

Figure 1.1 Distortion of the signals due to dispersion in different distances from the actuator. 


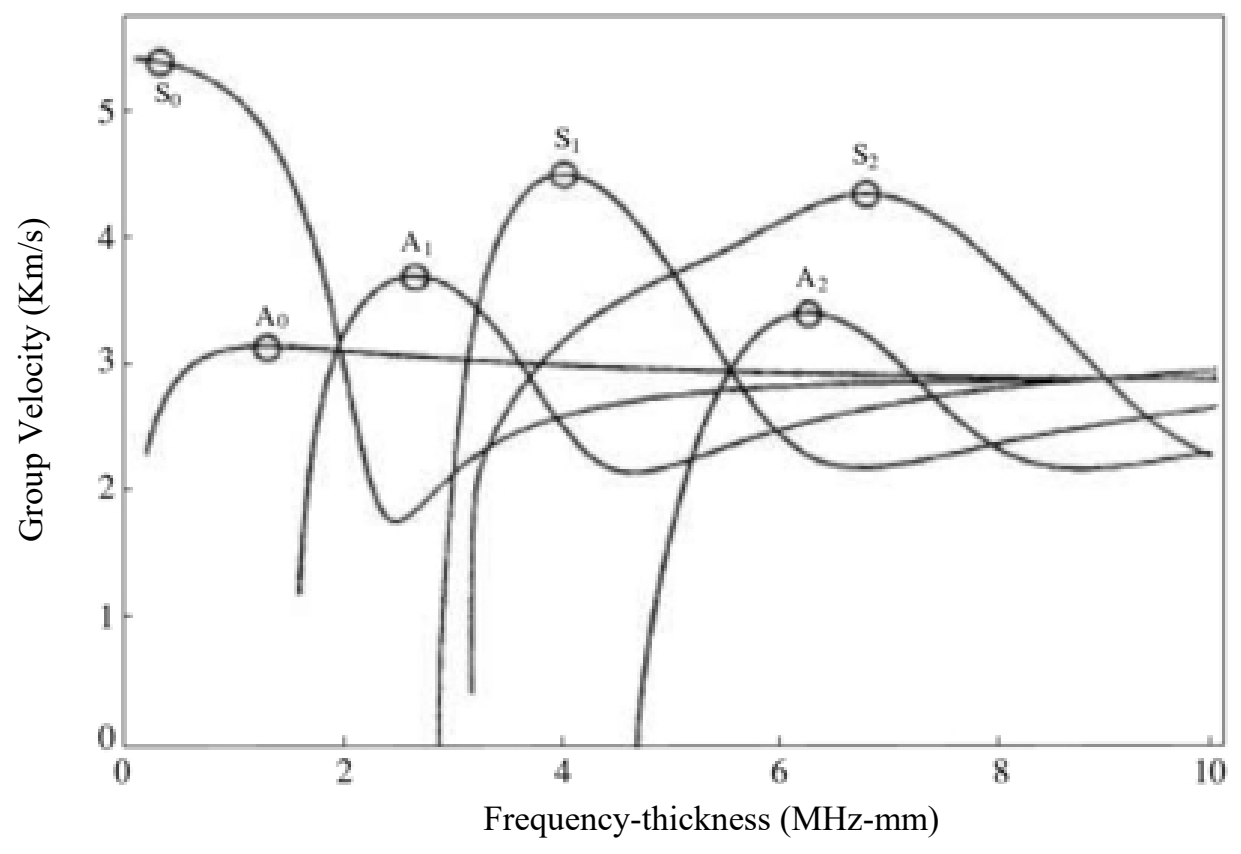

Figure 1.2 Dispersion curve for an aluminum plate [49].

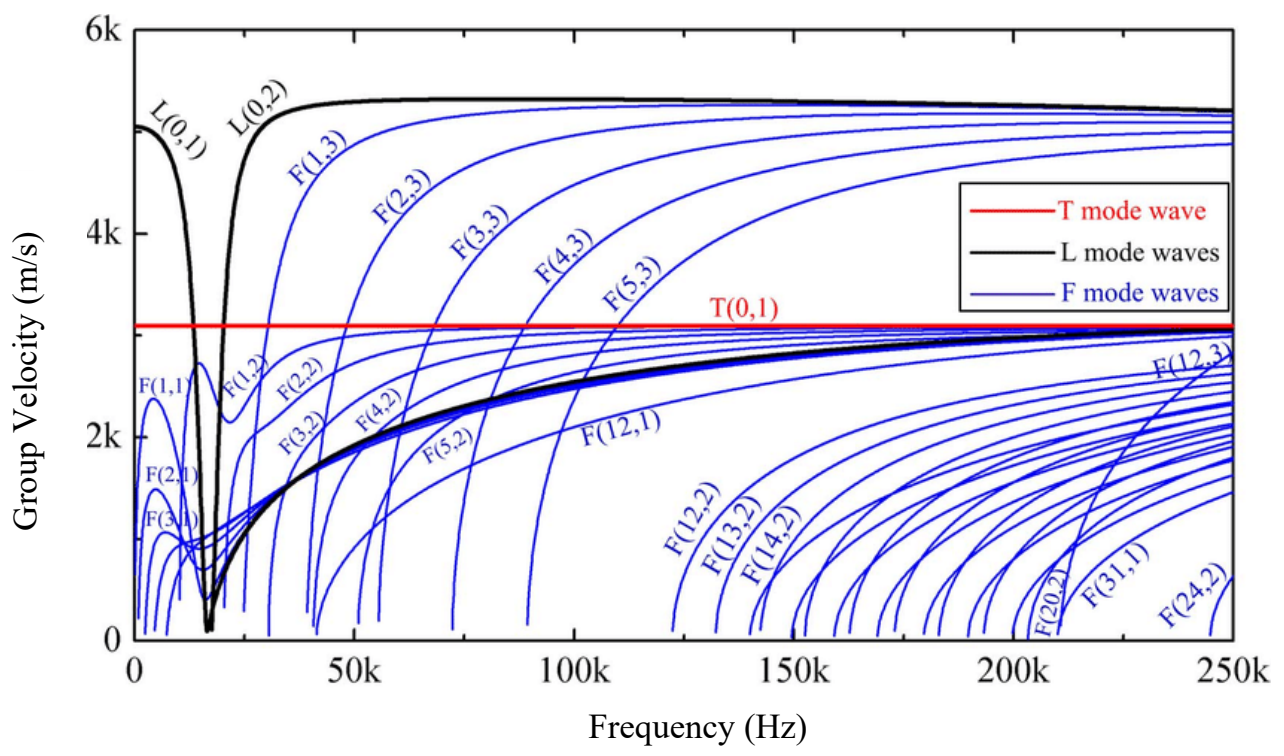

Figure 1.3 Dispersion curve for an aluminum pipe [50].

Another possible solution is to excite the structure with broad band frequencies [44]-[46]. In this approach, the analysis is mainly performed in the frequency domain since the response cannot be easily interpreted in the time domain. The well-known EMIS approach, 
traditionally, needs bulky and expensive analyzers to detect defects in such a way. Recently, some cheaper electronic chips are introduced that can lower the cost of such an approach [47], [48]. Yet, the impedance information that is used in this approach has a local nature and therefore high quantity of sensors are required for inspection of large structures.

The majority of available single frequency and wide range frequency approaches are based on monitoring linear characteristics, such as, time of flight, reflections from defects, energy transmission, and impedance information. The common problem of these methods is that their sensitivity to small defects such as fatigue cracks is low and such defects are usually detected when they reach about $80 \%$ of their fatigue life [6].

The newer nonlinear SHM approaches, monitor nonlinear characteristics of damaged structures. Nonlinear methods such as time reversal and nonlinear elastic wave spectroscopy (NEWS) methods like nonlinear resonant ultrasound spectroscopy (NRUS ) and nonlinear wave modulation spectroscopy (NWMS) show higher sensitivity to small defects [51]-[55]. However, they share three problems with linear methods. First, selecting the appropriate excitation frequency that fits a particular type of defect. Second, to date, sensors were the inevitable part of active monitoring methods and are used for collection of the response of a structure to a known excitation. Usually, a very good understanding of wave propagation in solid media and its interaction with defects is a prerequisite for the successful running of required tests. Third, sensors to be used in SHM must meet some minimum requirements:1) They have to be compatible with the performance of the host structure; 2) They must not adversely affect mechanical integrity of the host structure; 
3) They have to be capable of inspecting rather large areas of the host structure without moving; 4) They can be used in the rugged environment of in-service structures. To date, no sensor has all of these characteristics together at the same time. Also, a sensor which has all of these characteristics would not be cheap and therefore could not be used in high quantities for monitoring large structures. Finally, sensors themselves are prone to damage and degradation.

\subsection{Research Goals, Scope, and Objectives}

This study focuses on ultrasonic SHM approaches that monitor different aspects of guided waves' propagation and interaction with defects for detection and identification of damage. Sophisticated equipment and extensive computations are common parts of such SHM applications. Development and implementation of reliable, compact, and cost-effective (with sensor and sensor-free SHM) schemes are the main goals of the current study.

Objectives of the study are as follows:

- Providing theoretical justification for the validity of the surface response to excitation method through constitutive piezo electric equations.

- Implementing the surface response to excitation method for pipes in contact and non-contact modes.

- Developing nonlinear approaches based on the heterodyning effect for early detection of small cracks.

- Eliminating the need for sensors in monitoring processes through development of the very first sensor-free scheme by using the heterodyning effect. 
- Improving the localization of defects by mixing virtual and real beamforming approaches.

The dissertation is composed of six chapters. The current chapter contains the introduction and general discussions about SHM. Chapter 2 discusses the validity of the surface response to excitation (SuRE) method as a cheaper alternative to electro mechanical impedance spectroscopy (EMIS) approaches. The SuRE method is then applied for the detection and quantification of different severities of damage in pipes using contact and non-contact approaches. The thesis then proceeds with exploring different nonlinear methods in SHM and then introduces the heterodyning effect and novel sensor-less and with sensor approaches for early detection of damages for addressing the shortcomings of available methods in chapters 3 and 4 . Chapter 5 discusses the applications of array systems in SHM and then presents an improved phased array method for surface imaging and localization of multiple damages in plate-like structures. Finally, chapter 6 presents conclusions of the current study and suggestions for future studies. 


\section{CHAPTER 2}

\section{Surface Response to Excitation Method (SuRE)}

Active structural health monitoring methods are becoming increasingly popular for defect detection in hollow cylindrical structures. Different types of time and frequency domain based studies have been applied to examine the health of hollow cylindrical structures using guided waves. The Surface Response to Excitation (SuRE) method was originally developed for detection of defects and monitoring of loads applied on plate-like structures. In this chapter, the validity of the surface response to excitation method is discussed theoretically and the method is implemented experimentally for detection of both internal and external damages in pipes using contact and non-contact approaches.

\subsection{Introduction}

Structural health monitoring (SHM) of hollow cylindrical structures are of paramount importance in oil, gas, chemical, and petrochemical industries. Typically, the interior area of pipes cannot be easily accessed and monitored because of the geometry of the system, small size of the cross section, cost of the process, or presence of hazardous substances. The exterior area of pipes can also be hard to access and monitor due to insulation or special positioning of them. Thus, using conventional nondestructive methods for damage detection in long and complex systems of pipelines is a highly expensive, time consuming, and even sometimes an impossible process. Guided-waves based methods have found wide applications in SHM of hollow cylindrical shapes because of the fact that these waves can propagate for long distances with low attenuation inside the curved walls of pipe-like structures [33], [56], [57]. Therefore, the monitoring process can be performed by only 
having access to a few points on the surface of the structure and there is no need for complete removal of insulation.

Guided waves are mechanical waves that are guided by boundaries of a structure. It should be noted that while propagating in solid media they show different propagation velocities depending on the mode and frequency of excitation. It is very important to note that different wave propagation modes might be excited and exist at even one single frequency in the monitoring process. Therefore, a wave that consists of more than one mode deforms during its propagation, even in single frequency excitations. This is called dispersion and it makes the data interpretation and analysis in the time domain a troublesome task [58], [59]. In plate-like structures, only two main group of symmetric and antisymmetric modes exist. However, the three modes that exist in hollow cylindrical structures are longitudinal axially symmetric, torsional axially symmetric, and non-axially symmetric (flexural) modes [60]-[62]. Due to the existence of more propagation modes, defect detection in hollow cylindrical structures is more complicated when compared to plate-like structures.

The part of SHM methods that use a combination of actuators and sensors in the monitoring process are called active methods [63]-[65]. Actuators are used for controlled and systematic excitation of structures and sensors collect the response of a target structure to a known excitation. Instead, methods that do not utilize actuators and only use sensors for detection of defects are called passive methods [28], [29]. In linear active methods, the input to the system is known and the main focus is on the interpretation of the response. There are different types of techniques for excitation and collection of responses in active SHM approaches. Methods, such as pulse-echo [66], [67] and pitch-catch [68], [69] have 
mainly used guided waves for monitoring of hollow cylindrical structures in the time domain. Successful detection of defects in hollow cylindrical structures using these methods highly depends on appropriate selection of probing mode and frequency. That is to say, different wave modes do not show the same sensitivity towards a certain type of defect. For example, torsional modes can detect longitudinal defects better than longitudinal modes when those defects have a much lower width in comparison to their length.

Another group of active SHM methods are electro mechanical impedance spectroscopy (EMIS) based methods which use a broad frequency range for excitation [46], [70]-[72]. EMIS uses a single piezoelectric (PZT) element as both actuator and sensor. Since these methods use a broad frequency range for excitation they are sensitive to a wider variety of defects. That is, more different sizes and orientations of defects can be detected in a single monitoring process. It has to be noted that, in its original form, a costly EMI analyzer is required to extract useful data from signals that are being collected by a PZT sensor. EMIS based methods are index based, meaning that a damage index is calculated by comparing the impedance of the response of the target structure to a known excitation, at any time, with a reference baseline (acquired in a pristine state). That is, EMIS techniques can detect changes in the dynamic response of a structure and correlate that alteration with creation and growth of damage. There have been some efforts to substitute the bulky and expensive EMI analyzers, such as HP4294A, with miniaturized impedance measuring devices that are based on cheaper electronic chips and microcontrollers [47], [48]. While this helps to 
reduce the cost, due to the limited and local sensitivity of the process, online monitoring of a large area may require a large quantity of sensors.

The surface response to excitation (SuRE) method is another broad frequency range SHM method. In one way, it is similar to pitch-catch methods since it uses different PZT elements for excitation and reading. However, the SuRE method is a frequency domain based study and similar to EMIS method, it analyzes the frequency response of the structure in a broad frequency range. The broad frequency range excites multiple wave modes in the structure. This provides a means for the method to be highly sensitive to defects with different sizes, orientations and configurations. In the SuRE method, only the frequency response of the structure is obtained and used for monitoring the state of the health of a target structure. Typically, the appropriate excitation frequency range is selected to cover at least 20-30 peaks in the frequency response. Therefore, the appropriate range could be determined with a single test at any point for any new specimen.

In some applications, sensors cannot be attached to the surface of the structure for reasons, such as design considerations or high temperature. Using the SuRE method, instead of a second piezoelectric element, any contact or non-contact sensor may be used as long as the surface vibrations at the selected frequency bandwidth can be measured. The SuRE method eliminates the need for the EMI analyzer, it has high sensitivity to defects not in close proximity of sensor(s), and can work in noncontact mode. Therefore, SuRE can safely be considered as a cheaper alternative for EMIS based methods. The SuRE method was previously used for defect detection and localization in plate like structures, process performance monitoring, and for load monitoring purposes [73]-[76]. 
In this study, initially, theoretical justifications were provided for validity of the SuRE method. Then, the SuRE method was implemented for detection of internal and external damages in hollow cylindrical structures. Two main sets of experiments were designed to examine the efficiency of the SuRE method in detection of internal and external damages in pipes. Permanently bonded transducers were utilized for excitation and the responses of structures were collected in contact and non-contact approaches. Different lengths and depths of artificial damages were created and efficiency of the SuRE method in detection and quantification between different severities of damages were studied. The theoretical back ground, experimental setup, and results are discussed in the following sections.

\subsection{Theoretical Background \& Proposed Method}

In the SuRE method, typically, a PZT transducer is used as the actuator and excites the structure's surface in a broad frequency range, using sweep sine waves. The propagated waves are collected by one or more PZT elements where the mechanical energy of the propagated waves is transformed into electrical energy. The linear matrix form of the strain formulation of the constitutive model for a general one dimensional piezoelectric material is shown in equation (2.1). In this equation, $D$ is electrical flux density, $S$ is mechanical strain, $\varepsilon^{T}$ is permittivity in constant stress, $d$ is piezoelectric charge coefficient, $s^{E}$ is compliance in constant electrical field, $E$ is electrical field, and $T$ is mechanical stress [77]. Strain and electric displacement are taken as dependent variables.

$$
\left\{\begin{array}{l}
D \\
S
\end{array}\right\}=\left[\begin{array}{ll}
\varepsilon^{T} & d_{33} \\
d_{33} & s^{E}
\end{array}\right]\left\{\begin{array}{l}
E \\
T
\end{array}\right\}
$$


Global constitutive equations of a PZT transducer [78], in sweep type actuation and sensing, with a uniform distribution of mechanical and electrical properties, throughout the whole volume of the transducer can be represented by integration over the entire volume of the transducer:

$$
\left\{\begin{array}{c}
\tilde{V} \\
\tilde{f}
\end{array}\right\}=\frac{K_{a}}{C\left(1-k^{2}\right)}\left[\begin{array}{cc}
1 / K_{a} & -d 33 \\
-d 33 & C
\end{array}\right]\left[\begin{array}{c}
\tilde{Q} \\
\tilde{\Delta}
\end{array}\right\}
$$

Where $V$ is the voltage, $f$ is total force, $C$ is capacitance measured with no force, $K_{a}$ is stiffness when $V=0, k$ is electromechanical coupling factor, $Q$ is the total charge of electrodes of the transducer, and $\Delta$ is the total elongation. As can be seen from equation (2.2), mechanical waves which are generated in the actuator element by the sweep excitation produce a harmonic voltage in the sensor PZT element upon reaching to it. When a PZT transducer is bonded to the structure, mechanical impedance of the structure is coupled with the mechanical impedance of the PZT transducer and in a PZT transducer the mechanical and electrical impedances are coupled together. The electromechanical coupling factor $(k)$ of a PZT element can be calculated as shown in equation (2.3) [78]:

$$
k^{2}=\frac{d_{33}^{2}}{s^{E} \varepsilon^{T}}
$$

In the monitoring process, it is assumed that the PZT remains intact during the monitoring process while specific characteristics of the structure such as stiffness, damping, and dynamic mass of the structure change in the presence of damage. Because of the existing coupling between the mechanical impedance of the structure and electrical impedance of the PZT sensor element, defects alter the output voltage of the sensor into a fixed excitation 
depending on their distance from the sensor. That is to say, if the damage is not close enough depending on the applied excitation frequency then the change in the output voltage due to the change of the mechanical impedance of the structure cannot be a good indicator of the state of the health of the structure. Although, it is very crucial to notice that the waves that are received in the sensor PZT, which are produced with similar excitation parameters, are not the same before and after damage. This is due to the change in scattering, diffraction, attenuation, or propagation behavior of waves in presence of damage.

In summary, defects change the output voltage of a PZT sensor subjected to a constant input excitation. A part of the change in the output voltage is related to the change in the mechanical impedance of the structure. However, this mechanical impedance change is a local parameter and highly depends on the applied excitation frequency. The higher the excitation frequency the more limited the area that can be efficiently sensed based on alteration of the mechanical impedance. Another reason for the voltage change is the diffraction, scattering, and deviation of propagating waves caused by damage on their path of propagation from the actuator to the sensor. The latter is more of a global parameter, meaning that damage can be detected even if it is relatively far away from the PZT sensor and not in a close distance of the sensor PZT. It should be noted that waves with different modes and frequencies show different sensitivities to similar types of defects. Therefore, by sweep excitation, multiple modes and frequencies are excited and exist together which makes the SuRE method sensitive to damages with different types of characteristics. Thus, by using a sweep sine excitation and employing two different elements for excitation and sensing, an accurate and comprehensive assessment of the structure can be achieved. 
The dynamic response of a structure to a known sweep excitation can be obtained from the Fast Fourier Transform (FFT) of sensor signals. The response of a structure into a fixed excitation, in sensor locations $\mathrm{j}=1, \ldots, \mathrm{n}$, before and after damage can be shown with matrices such as the ones that are shown in equations (2.4) and (2.5), respectively. $B$ and $D$ indicate the baseline and damage states, $m$ is the number of scanned frequencies, and $n$ is the number of sensor locations in the network. As long as there is no change in the state of the health of structure, its response to a fixed excitation remains the same. However, the frequency response drastically changes when damage occurs or external loads are applied on a target structure.

$$
\begin{aligned}
& V_{B}(f)=B_{m * n} \\
& V_{D}(f)=D_{m * n}
\end{aligned}
$$

In the present study, at each sensor location, the sum of squares of differences (SSD) of the response at any time with respect to a baseline response is used to quantify the severity of damage in the SuRE method. SSD of the frequency response matrix of a damaged part with respect to the pristine structure (intact part) is calculated and shown in equation (2.6).

$$
S S D_{j=1, \ldots, n}=\sum_{i=1}^{i=m}\left(B_{i j-} D_{i j}\right)^{2}
$$

To summarize, the SuRE method is an index based method and in addition to the current response of the structure to a fixed excitation, it requires the response of the structure to the same excitation in an intact state to calculate the damage index and quantify the level of damage. 


\subsection{Experimental Setup}

\subsubsection{Detection of defects using bonded piezoelectric transducers}

An aluminum pipe having the dimensions, $711.2 \mathrm{~mm}$ (length), $20 \mathrm{~mm}$ (outside diameter) and $1.5 \mathrm{~mm}$ (wall thickness) was used as the test specimen. Two wooden supports with the inner diameter of $37 \mathrm{~mm}$ were used to hold the pipe in position. A $10 \mathrm{~mm}$ disk-shaped piezoelectric transducer was used as actuator and was permanently bonded to the surface of the pipe in the middle of the specimen. The actuator PZT was excited by a 60 volt continuous sweep sine wave in the range of $20-250 \mathrm{kHz}$. Four other PZT elements were used as sensors and permanently attached to the surface of the pipe.

The efficiency of the SuRE method in detection of internal and external damages, in contact mode, was studied through the creation of internal and external longitudinal cuts with a milling machine. Four different sets of experiments were carried out to separately study the effects of changes in the length and depth of internal and external damage in pipes. A milling tool with a diameter of $4.76 \mathrm{~mm}$ was used to create all cuts. To study the damage identifying ability of the SuRE method, the depth and length of the cuts were increased and studied in three steps. To study the effect of length, the length of the internal and external longitudinal defects was increased from $20.93 \mathrm{~mm}$ to $62.8 \mathrm{~mm}$ in the increments of 20.93 $\mathrm{mm}$. To study the effects of the depth of cut, the length of cut was kept constant and equal to $62.8 \mathrm{~mm}$ while the depth of the external and then internal longitudinal defects was increased in steps of $0.375 \mathrm{~mm}$, resulting in cuts with the same length but depths of 0.375 $\mathrm{mm}, 0.75 \mathrm{~mm}$, and $1.125 \mathrm{~mm}$. 
Responses of the pipe to the excitation were monitored at four different sensor points along the axis of the pipe using the four permanently mounted PZT elements. Before each experiment was conducted a "baseline" response of the pipe was obtained to be used as a baseline. The response signals were collected with a $10^{6}$ sample/s sampling rate by using a DT9832A data acquisition card. 4096 points size Fast Fourier Transform (FFT) were calculated to get the FFT of the acquired signals. The collected data was in the form of voltage-frequency curves. The schematic of the experimental setup used in this experiment is shown in Figure 2.1.

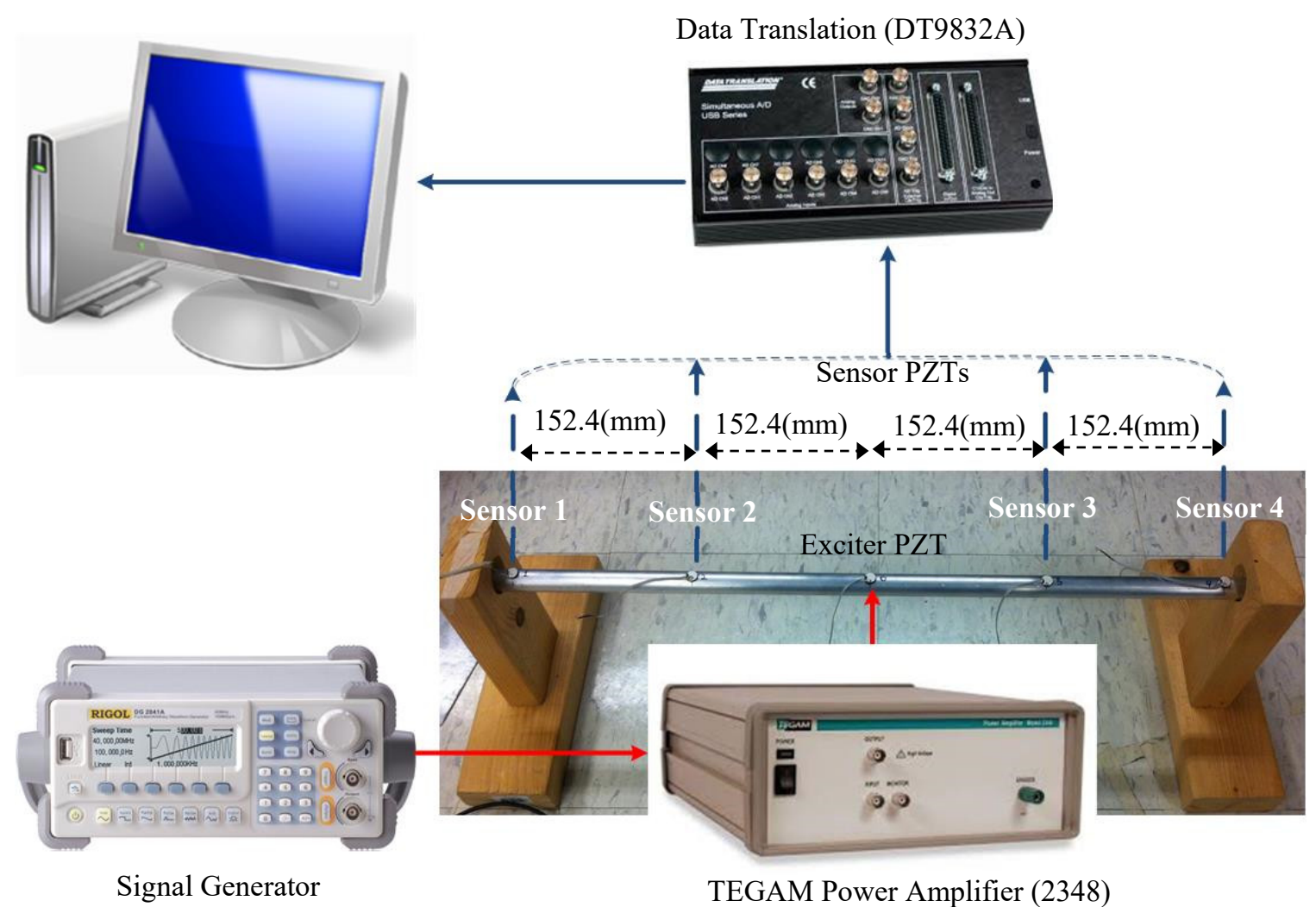

(Rigol DG1022)

Figure 2.1 Experimental setup for implementation of the SuRE method in contact mode. 


\subsubsection{Detection of defects in noncontact mode}

The purpose of the noncontact experiments is to demonstrate a non-contact SHM strategy for pipes with minimum dependency on the selection of proper excitation modes and frequencies. To achieve this, the SuRE method is implemented in noncontact mode using a scanning laser vibrometer. The performance of the SuRE method is evaluated when axisymmetric and non-axisymmetric defects exist on the surface of an aluminum pipe. Artificial longitudinal and circumferential defects were created on the aluminum pipe and their severities increased in three increments.

To validate the SuRE method in non-contact mode, an experimental setup was assembled that focused on evaluating an aluminum pipe having the dimensions, $226 \mathrm{~mm}$ (length), 26 $\mathrm{mm}$ (outside diameter) and $3 \mathrm{~mm}$ (wall thickness). Two metallic supports with plastic bushes with the inner diameter of $37 \mathrm{~mm}$ were used to hold the pipe in position. A $15 \mathrm{~mm}$ disk-shaped piezoelectric transducer was permanently bonded to the surface of the pipe section and was excited by a 20 volt continuous sweep sine wave in the range of $20-250$ $\mathrm{kHz}$. Initially, it was aimed to detect the presence of axisymmetric and non-axisymmetric damage and then to identify the increase in the severity of each damage. In order to represent axisymmetric damage, an artificial circumferential cut was created with three different depths. Then, a non-axisymmetric longitudinal cut was created and the length and the depth varied in three different levels.

Response of the pipe to the excitation in the un-damaged ("baseline") state and after each increase in the severity of the damage were monitored at eight different points along the axis of the pipe using a Polytec 3D Laser Scanning Vibrometer PSV400. The laser 
vibrometer signals were collected with a $10^{6}$ sample/s sampling rate by using a DT9832A data acquisition card. 4096 points size Fast Fourier Transform (FFT) were calculated to get the FFT of the acquired signals. The collected data were in the form of voltage-frequency curves. The schematic of experimental setup used in this experiment is shown in Figure 2.2 .

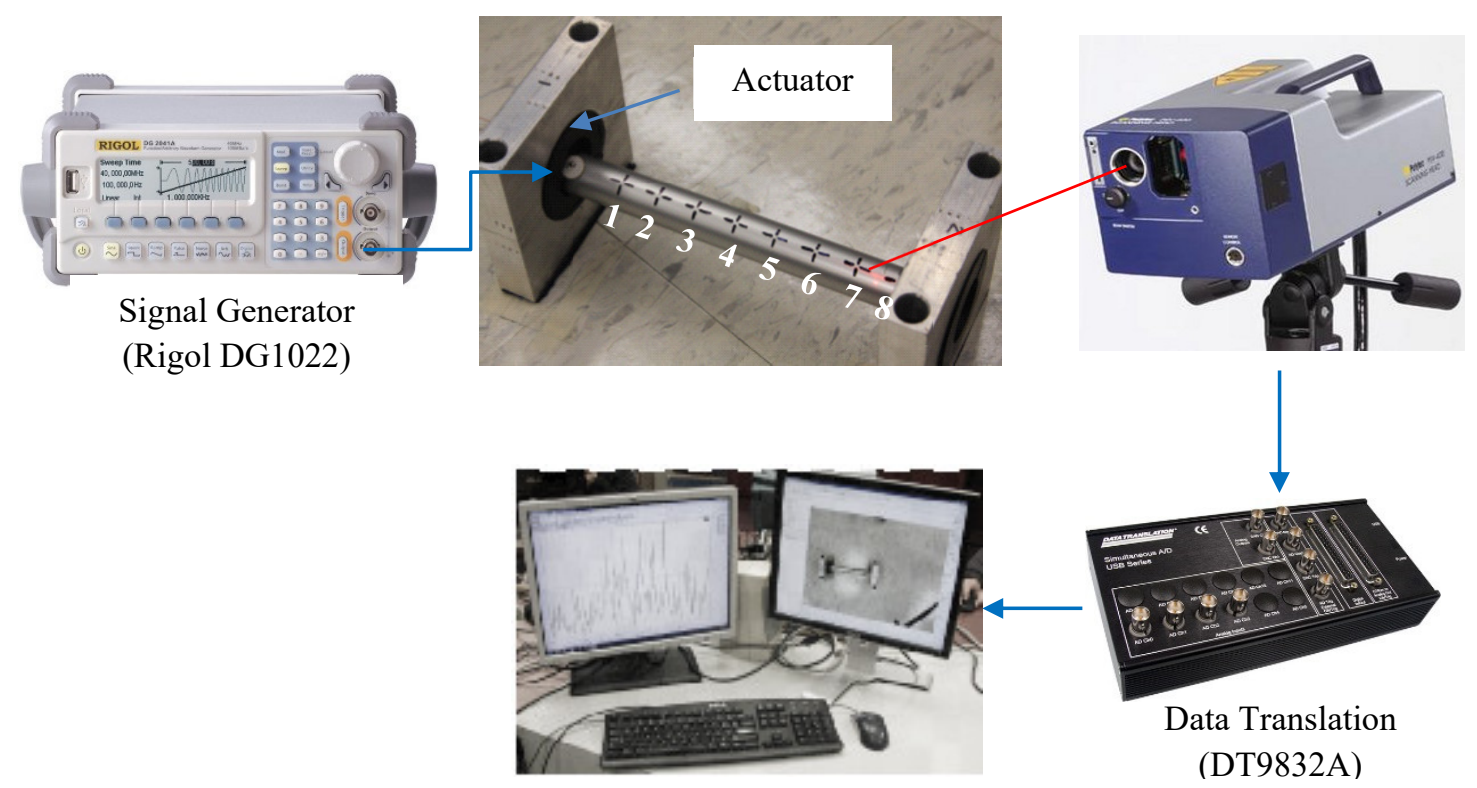

Figure 2.2 Experimental setup for implementation of the SuRE method in non-contact mode.

\subsection{Results and Discussion}

\subsubsection{Detection of defects using bonded piezoelectric transducers}

Length and depth of internal and external defects were changed on the pipe to evaluate the performance of the SuRE method. To show the negligible effect of changes due to boundary condition and other environmental parameters on SSD damage index, two baseline responses were acquired prior to the creation of each damage. The initial baseline responses were acquired from the damage free part at all sensor locations. The test-piece 
and stands were then completely disassembled and reassembled before a second set of baseline responses was acquired at all sensor locations. The SSD values acquired from the two sets of baseline responses provide a basis to separately compare the effect of damage and benign experimental changes on the SSD damage index. Performance of the SuRE method in the detection of internal and external damages in contact mode is discussed in following. In each test, three different responses were acquired and the average response of the pipe was used in the analysis.

\subsubsection{External Defects}

A longitudinal external damage with the depth of $0.75 \mathrm{~mm}$ and length of $20.93 \mathrm{~mm}$ was created between sensor two and the exciter, starting in $41.8 \mathrm{~mm}$ from sensor two. Then, the length of cut was increased toward the exciter in two more steps of $20.93 \mathrm{~mm}$. The three artificial damages are shown in Figure 2.3. Comparisons between initial baseline responses and responses after the creation of each damage at sensor location one are shown in Figure 2.4. As can be seen from Figure 2.4, the response of the pipe to the excitation is just about as it was prior to damage creation. However, the responses drastically change after the creation of each damage.

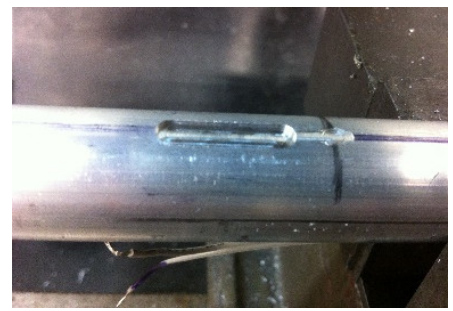

a) 22.93

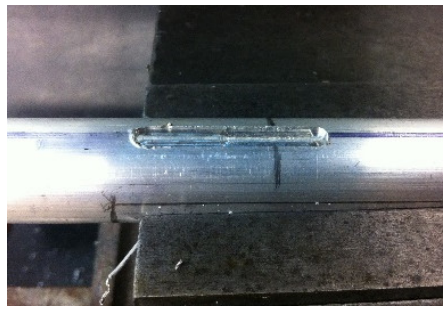

b) 45.86

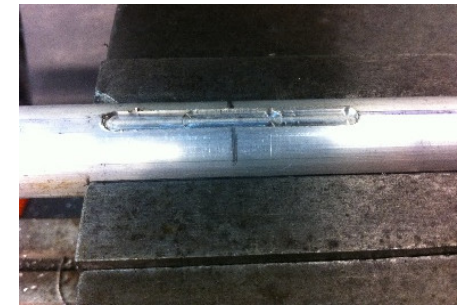

c) 68.79

Figure 2.3 External longitudinal damages with the same depth of $0.75 \mathrm{~mm}$ and length of a) $22.93 \mathrm{~mm} \mathrm{~b}) 45.86 \mathrm{~mm} \mathrm{c)} 68.79 \mathrm{~mm}$. 

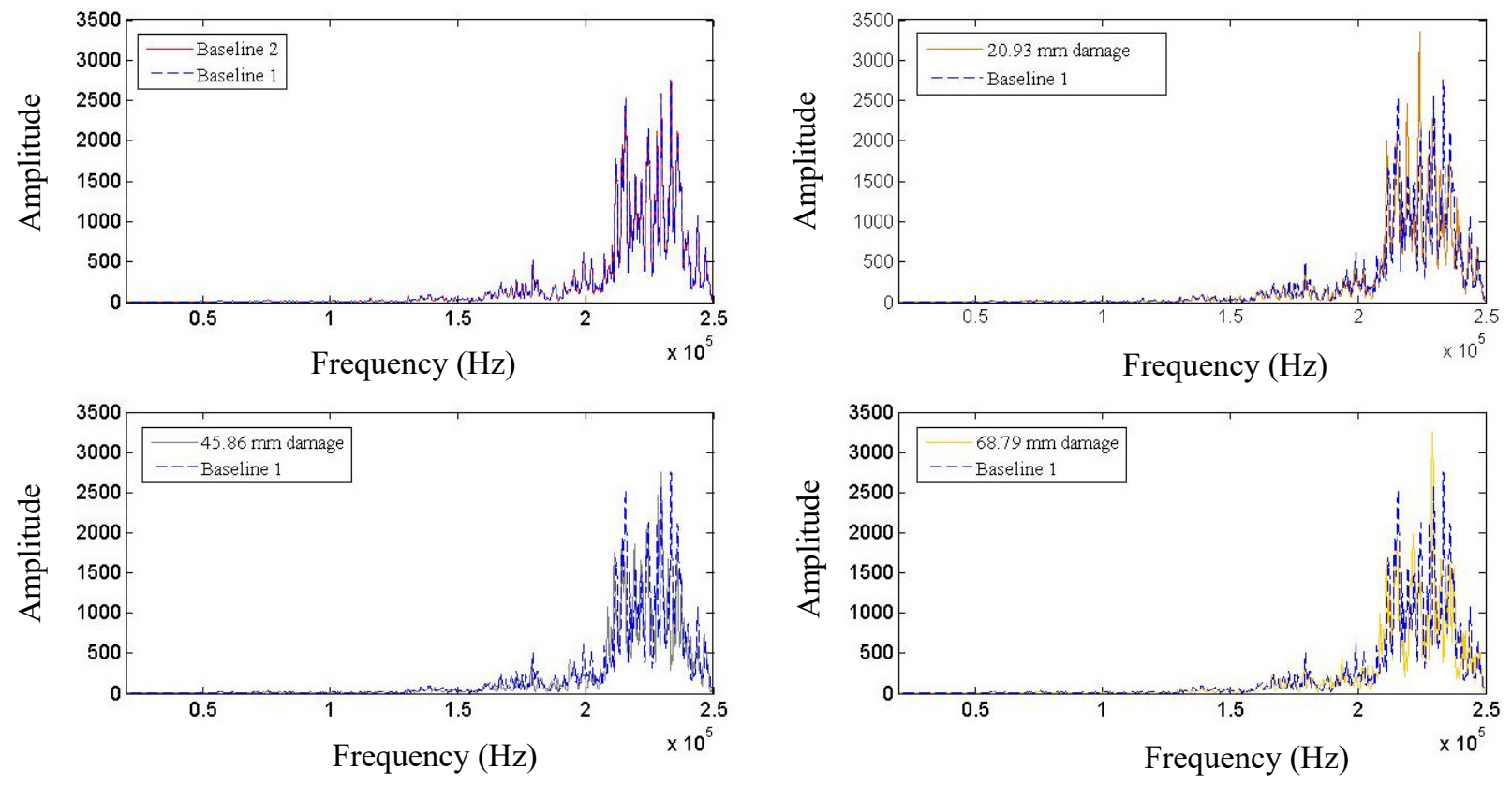

Figure 2.4 Comparison between initial baseline response and responses obtained at sensor location one after different damage lengths.

To quantify the difference between different levels of damages, SSD of the responses were calculated with respect to the initial baseline responses at all sensor locations and presented in Figure 2.5. As can be seen from the figure, the SSD values at all sensor locations, in the pristine condition, are insignificant when compared to the SSD values obtained after the creation of artificial damages. That is to say, the changes in the values of SSD of the second baseline with respect to initial baseline (smallest column in blue), is negligible in comparison to the change after the creation of damages. It should be noted that, the SSD damage index increases with each increase in the severity of the damage at all sensor locations. This indicates that the SuRE method is insensitive to benign changes of boundary conditions but it is very sensitive to the presence of an external damage in the structure. 


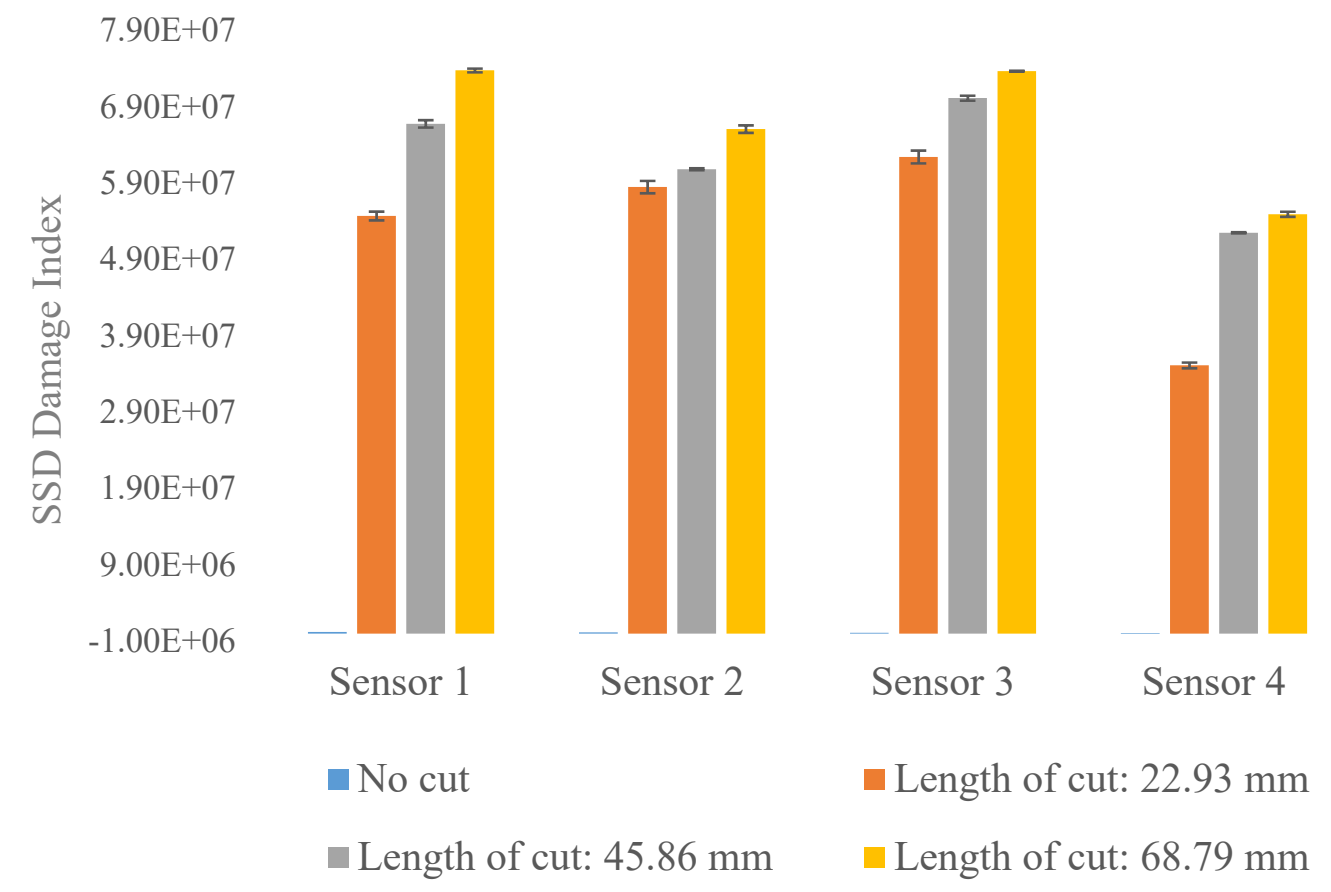

Figure 2.5 SSD damage index values before and after the creation of the external longitudinal damages at all sensor locations.

In addition to the defect growing in the axial direction, the sensitivity of the SuRE method to defects growing in the radial direction is also studied. A longitudinal cut with a constant length of $68.79 \mathrm{~mm}$ was created between the exciter and sensor number three, starting in $41.8 \mathrm{~mm}$ from the exciter. The depth of this cut was increased from $0.375 \mathrm{~mm}$ to 1.125 $\mathrm{mm}$ in steps of $0.375 \mathrm{~mm}$. The responses of the pipe specimen to the sweep excitation was acquired before and after the damages. The SSD values of the FFT of the responses with respect to the baseline response are shown in Figure 2.6. As can be seen from the figure the SuRE method was able to quantify the difference between depths of damages at all sensor locations. 


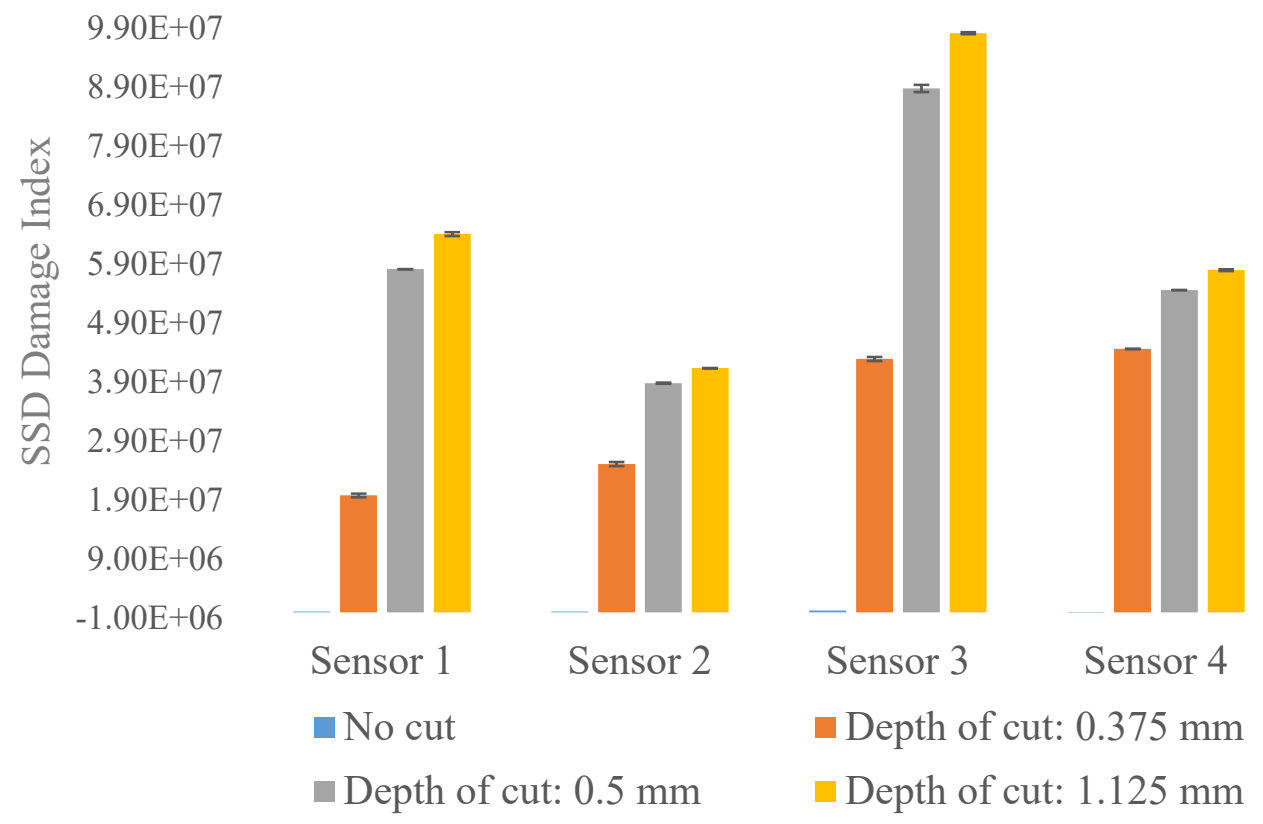

Figure 2.6 Damage index values at four sensor locations before and after the increases in the depth of the external longitudinal damage with constant length of $68.79 \mathrm{~mm}$.

\subsubsection{Internal Defects}

To show the efficiency of the SuRE method in detection of defects in different locations of pipes, internal damages with geometrical dimensions similar to the external damages were produced on the back side of the pipe. To create the internal damage a part of surface of the pipe with width of $10 \mathrm{~mm}$ and length of $80 \mathrm{~mm}$ was completely removed with milling process to create an access slot. Similar to external defect tests, two baselines were acquired. The first set of baseline data was acquired right after creation of the opening while the second set of baselines was collected one day later and after a complete disassembly and reassembly of the experimental setup. Both baselines were obtained after creation of the access slot. Therefore, the SuRE method now detects the changes in the dynamic response caused by the internal damages. 
To determine the effectiveness of the SuRE method in the detection of internal damages growing in the axial direction, an internal longitudinal cut with the same geometrical dimensions of the external longitudinal cut was created. The length of the internal damage was increased in the same three steps as the external longitudinal damage and are shown in Figure 2.7. The SSD of the FFT of the responses of the pipe to the excitation after creation of internal damages with respect to the initial baseline at all sensor locations are shown in Figure 2.8.

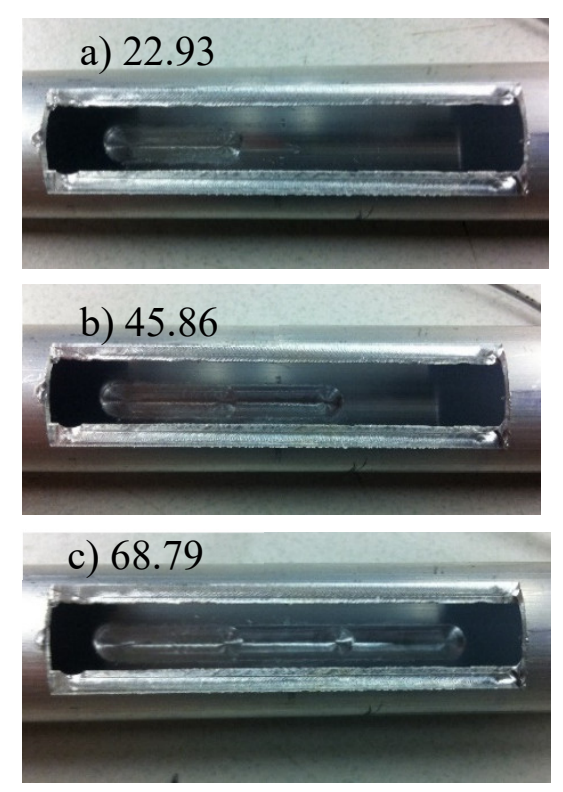

Figure 2.7 Internal longitudinal damages with the fixed depth of $0.75 \mathrm{~mm}$ and length of a) $22.93 \mathrm{~b}) 45.86 \mathrm{~mm}$ c) $68.79 \mathrm{~mm}$.

Moreover, the efficiency of the SuRE method in detection of internal damages that are growing in the depth is studied. A $68.79 \mathrm{~mm}$ cut with the depth of $0.375 \mathrm{~mm}$ was created and later its depth increased to $0.75 \mathrm{~mm}$ and $1.125 \mathrm{~mm}$. Like other experiments before creation of the damages two different sets of baseline were acquired. Values of the SSD damage index in the presence of damages with varying depths at all sensor locations are shown in Figure 2.9. 


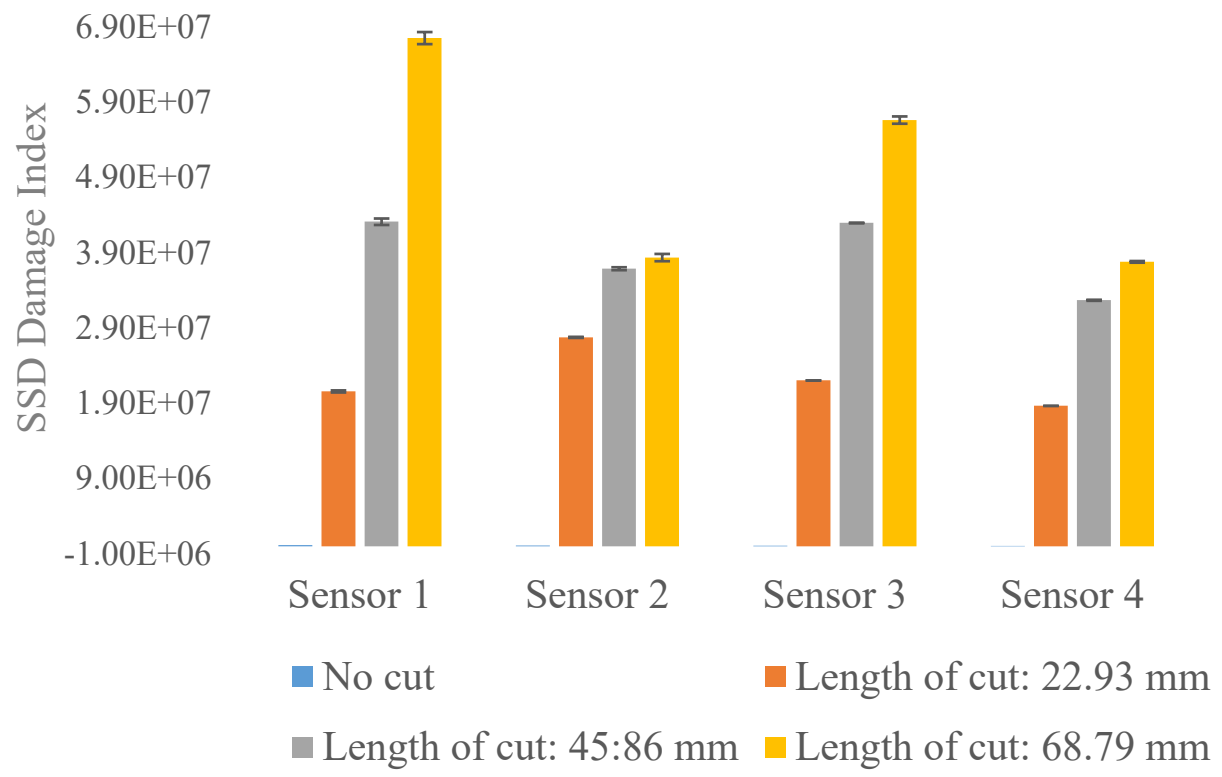

Figure 2.8 SSD damage index values at four sensor locations before and after the increases in the length of the internal longitudinal damage.

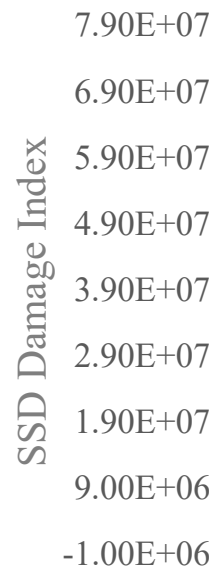

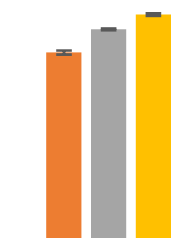

Sensor

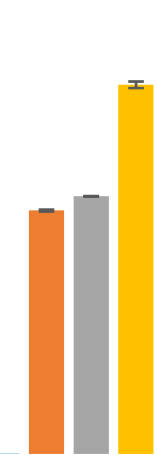

Sensor 2

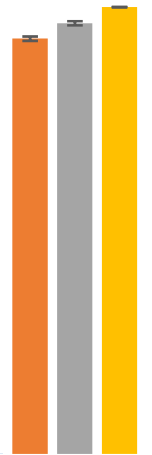

Sensor 3

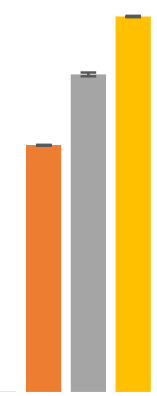

Sensor 4

- No cut

Depth of cut: $0.375 \mathrm{~mm}$

Depth of cut: $0.75 \mathrm{~mm}$

Depth of cut: $1.125 \mathrm{~mm}$

Figure 2.9 Damage index values at four sensor locations before and after the increases in depth of the $68.7 \mathrm{~mm}$ length longitudinal damage. 
From Figure 2.8 and Figure 2.9 can be seen that the SSD damage index for damages growing in axial and radial directions are different and vary with sensor location. However, the SSD damage index continuously increased along with the severity of the damage, for both types of damages, at all sensor locations.

\subsubsection{Detection of defects in noncontact mode}

Two different types of defects were created on the pipe to evaluate the performances of the SuRE method in noncontact mode. The artificial longitudinal and circumferential defects were created by using a milling machine with a $4.76 \mathrm{~mm}$ diameter end mill. The length of the non-axisymmetric longitudinal defect was increased from $40.84 \mathrm{~mm}$ to $81.68 \mathrm{~mm}$ in increments of $20.42 \mathrm{~mm}$. Additionally, the depths of the circumferential and longitudinal defects were increased in $0.5 \mathrm{~mm}$ steps.

Initially, it was important to show that benign environmental and experimental changes, such as temperature variations and slight changes of pipe position on supports, have negligible effect on the acquired response. For that purpose, the frequency response of the test pipe to the excitation was obtained at the same location on separate days. Prior to acquiring the data, the experimental setup was completely disassembled and then reassembled and the experiments were repeated. Also, three different responses of the system at the same location were acquired and the average response of the pipe was used in the analysis. As can be seen in Figure 2.10, there is minimal difference between the responses acquired in the two sets of tests. Also, for better demonstration of this high repeatability and reliability of experimental procedure, a part of the frequency response between 100$120 \mathrm{kHz}$ is magnified and shown in the figure. 


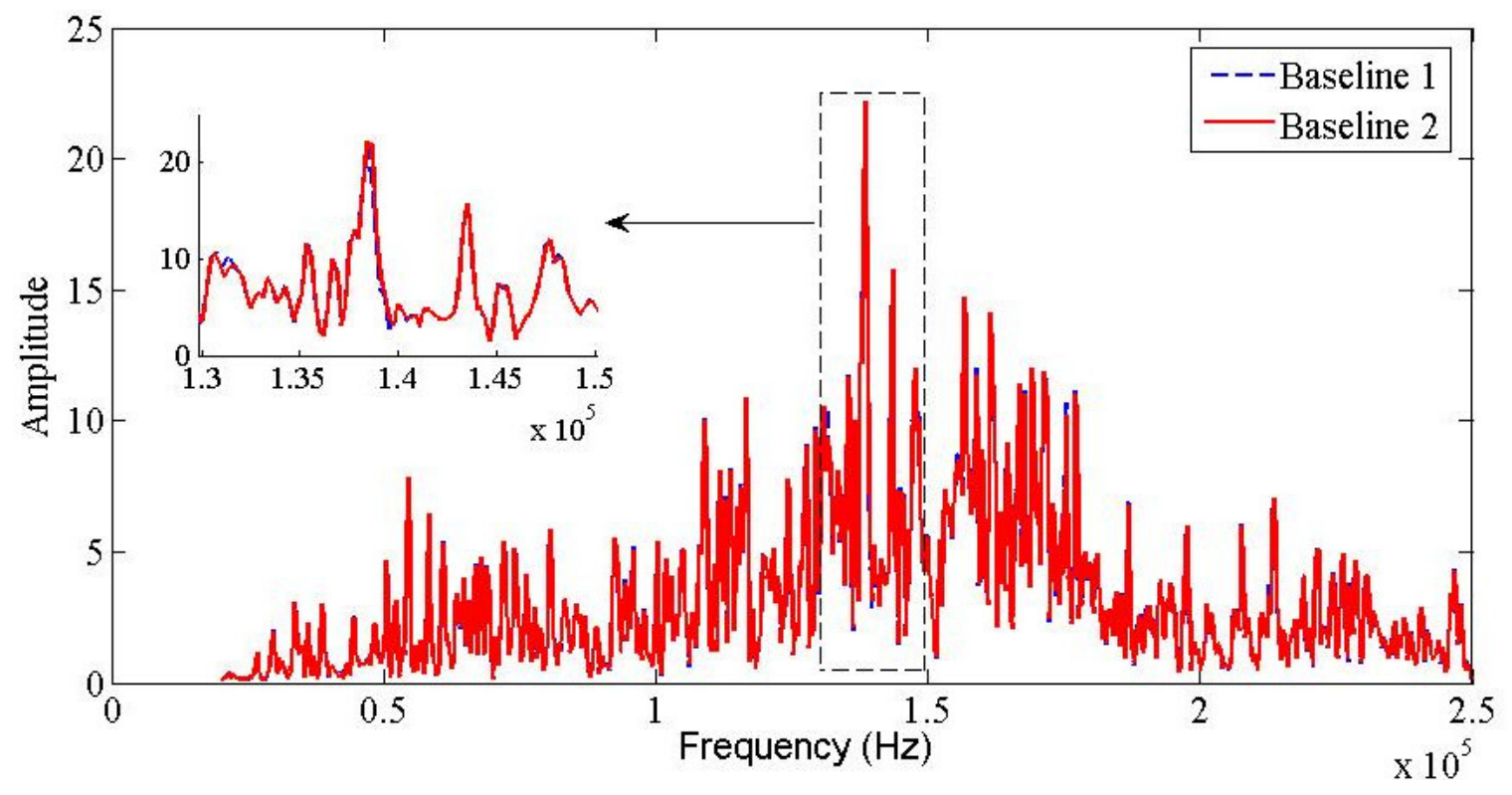

Figure 2.10 1st Baseline spectrum vs. 2nd Baseline spectrum.

\subsubsection{Circumferential Defect}

The initial depth of the complete circumferential cut was $1 \mathrm{~mm}$. The depth was then increased to $1.5 \mathrm{~mm}$ and $2 \mathrm{~mm}$ in subsequent tests. As expected, the frequency response changed dramatically upon introducing the defects. For instance, the frequency responses of the pipe after different levels of the circumferential damage at sensor location 1 are shown in Figure 2.11.

The dynamic responses of the pipe at eight sensor locations were acquired before and after increasing the damage depth. By using equation (2.6), the SSD values were calculated with respect to baseline frequency spectrums and the results at all sensor locations are shown in Figure 2.12. In addition, in order to provide a basis for comparison between the changes of damage indexes in a healthy and damaged structure, SSD values for two different sets of baselines (no damage) are also provided in Figure 2.12. 
(a)

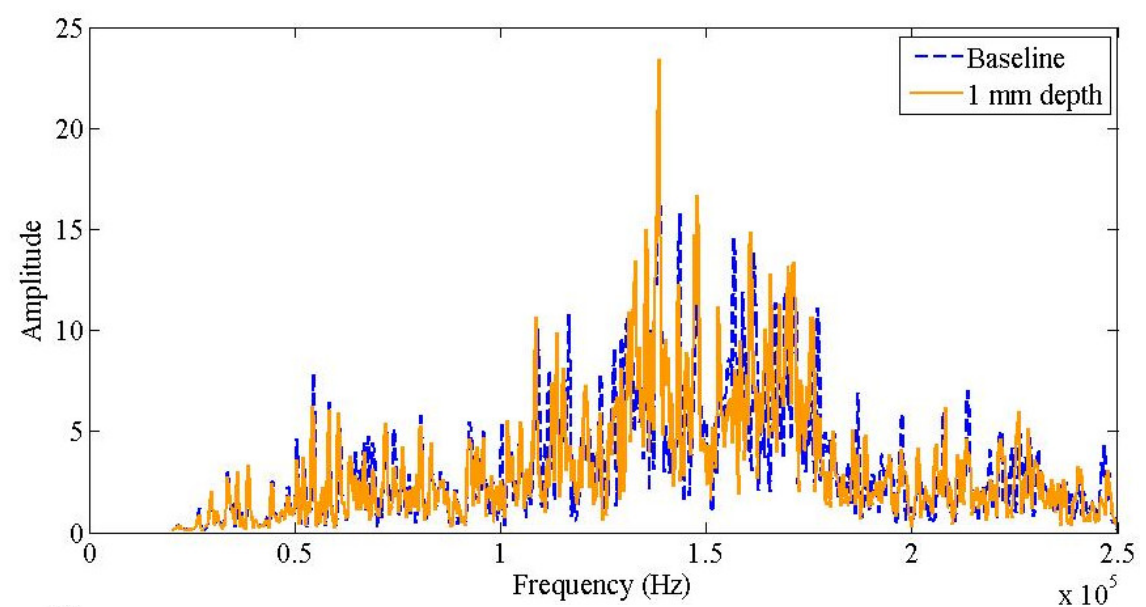

(b)

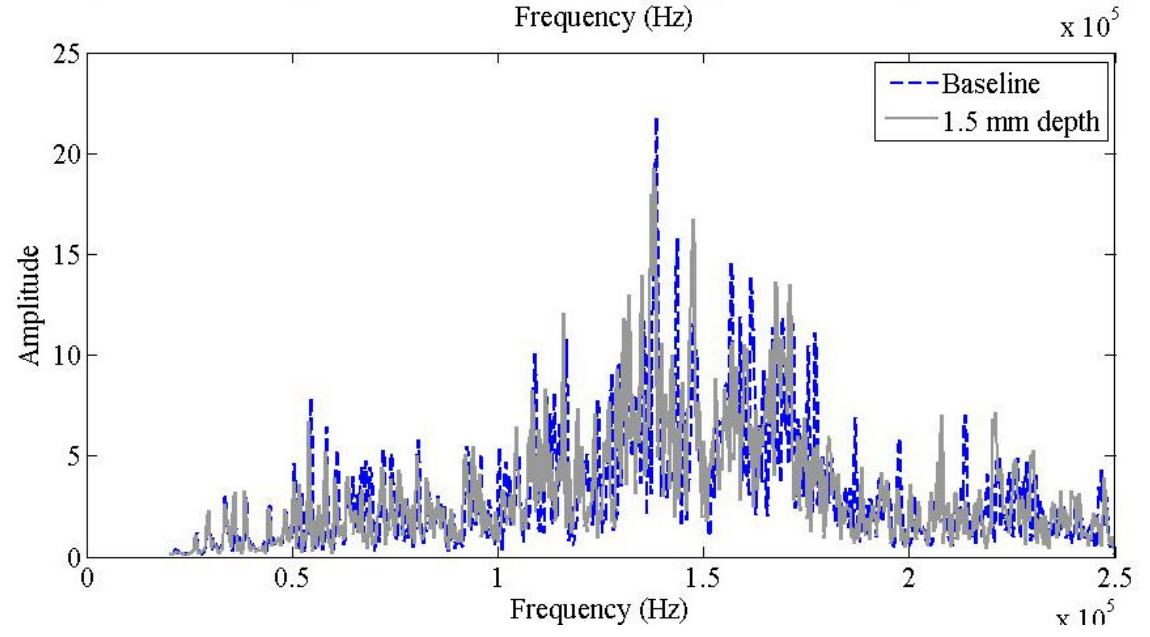

(c)

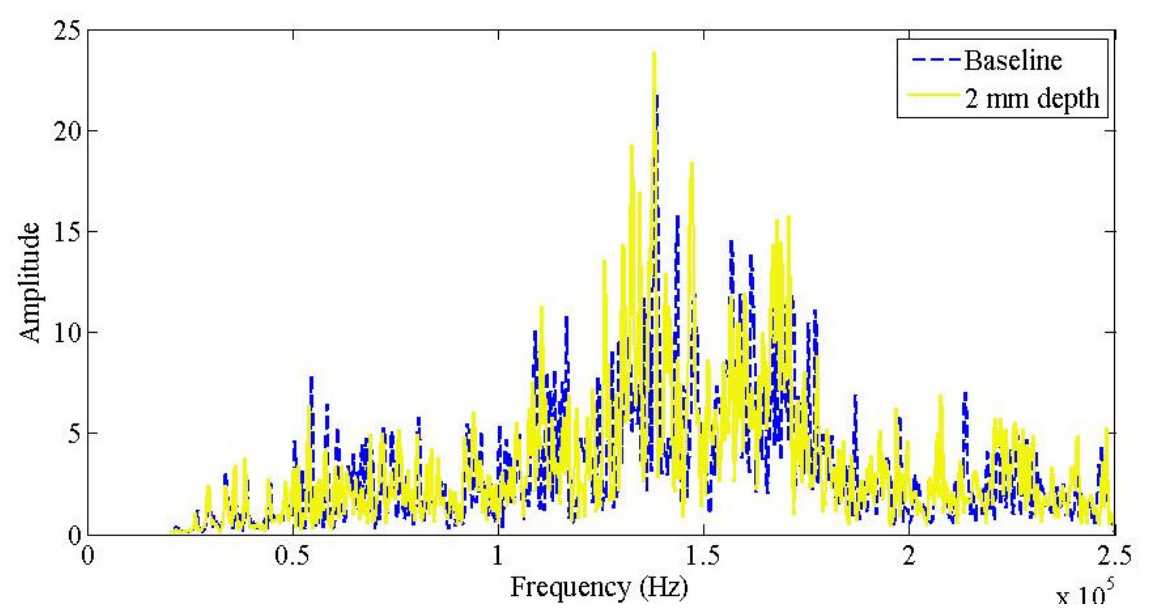

Figure 2.11 Frequency responses of the pipe after different levels of the circumferential damage at sensor location 1. 


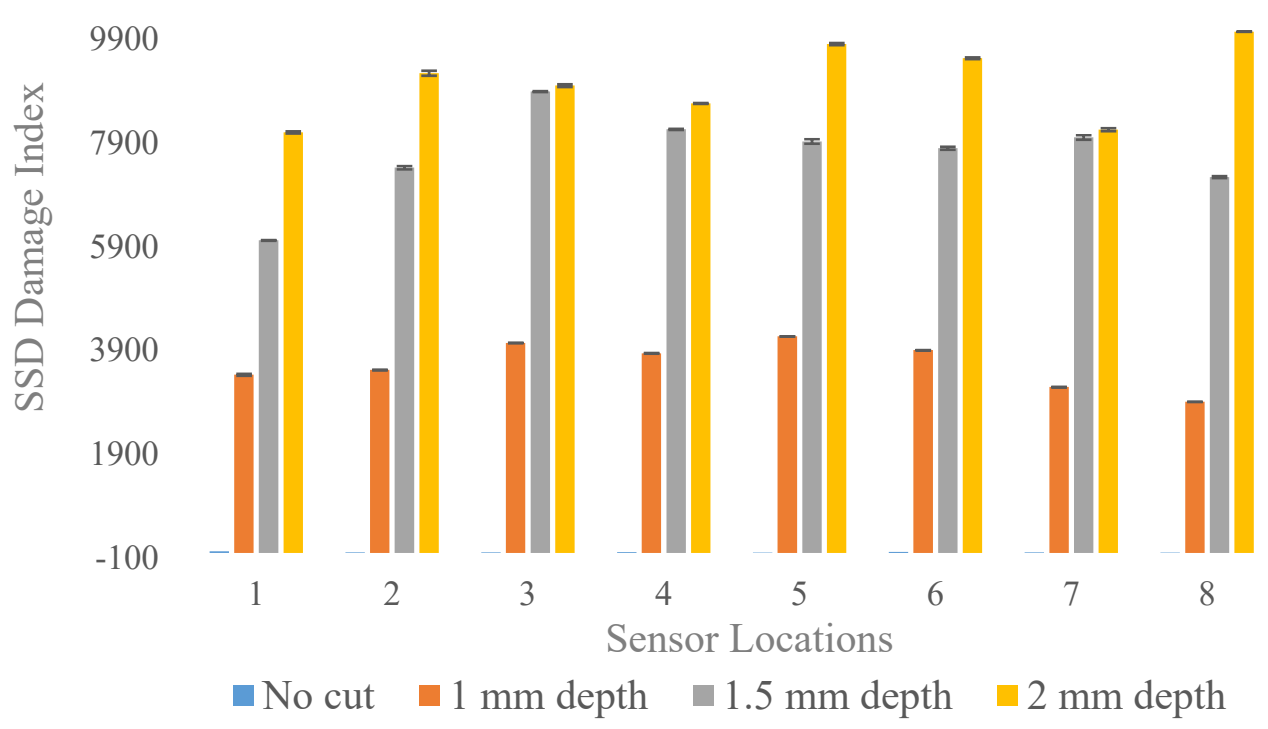

Figure 2.12 SSD damage index values at eight sensory points before and after the increases in depth of the circumferential damage.

Figure 2.12 shows that the SSD values increased when the depth of the damage was increased. The SuRE method successfully distinguished the three damage states at all sensing points.

\subsubsection{Longitudinal defect}

A second set of tests was conducted to investigate the effectiveness of the SuRE method for detection of the longitudinal defects in non-contact mode and distinguishing the severity of the damage. The response of the pipe to the excitation in a pristine state was studied at the eight sensing locations. The same tool, which was used for creating the circumferential damages, was then used to create the longitudinal damages. The length of the initial longitudinal defect was equal to $40 \mathrm{~mm}$. The length was then increased to $60 \mathrm{~mm}$ and $80 \mathrm{~mm}$. The depth of the cut was held constant and equal to $0.5 \mathrm{~mm}$ for all three lengths. 
The SSD values were again determined using equation (2.6) and by comparing the test cases with a reference collected when the pipe had no defects. The SSD values referring to the different levels of damage at each test point are presented in Figure 2.13. Similar to the circumferential defects, the SSD values increased at all sensing locations when the length of the damage was increased.

In addition, the performance of the SuRE method was evaluated when a linear damage was created with three different depths. The same tool was used to create the $100 \mathrm{~mm}$ length linear damage with $1 \mathrm{~mm}, 1.5 \mathrm{~mm}$ and $2 \mathrm{~mm}$ depths. The SSD values are plotted in Figure 2.14. The SSD values increased drastically in Figure 2.14 when the linear defect was created and its depth was increased. A similar trend was observed at all sensing points. The study verified that the SuRE method is also sensitive to the wall thickness loss in the longitudinal direction.

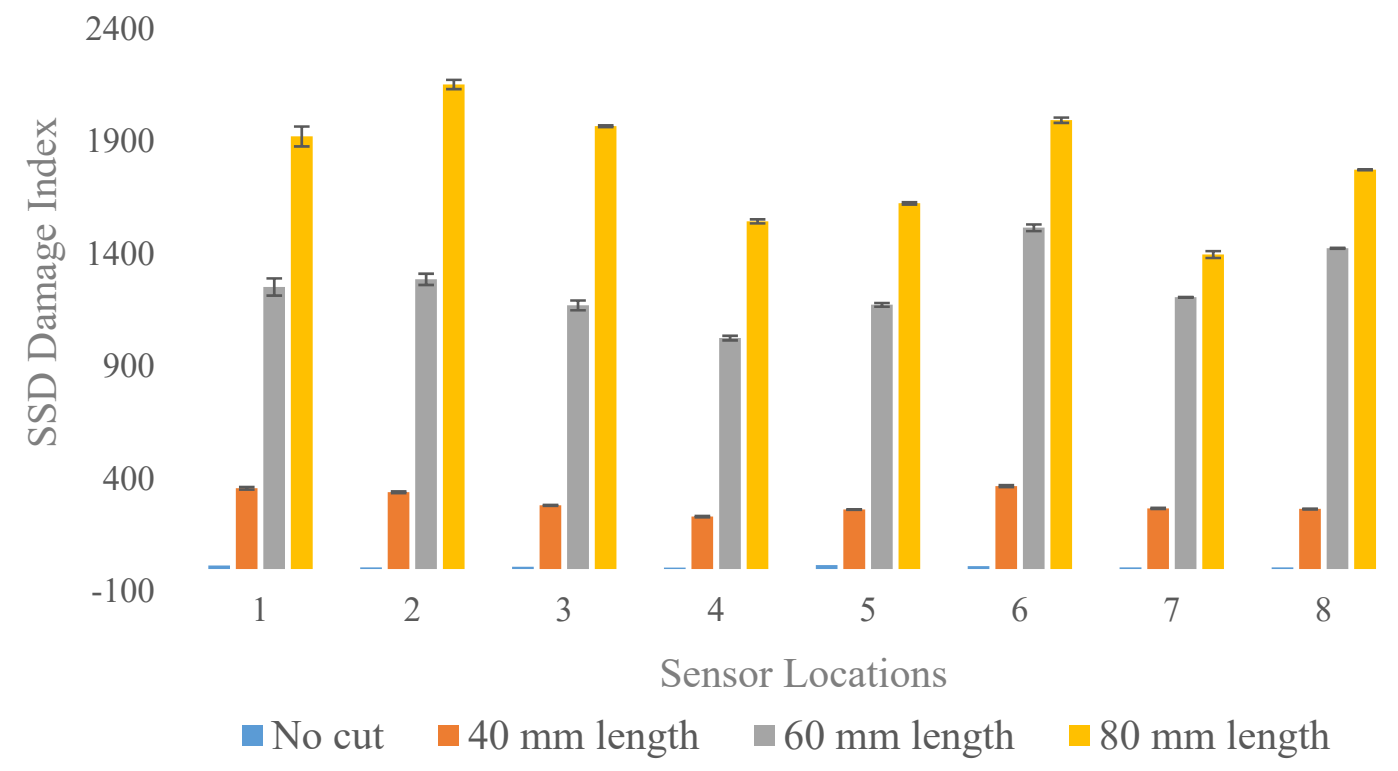

Figure 2.13 SSD damage index values at eight sensor locations before and after the increases in the length of the longitudinal damage. 


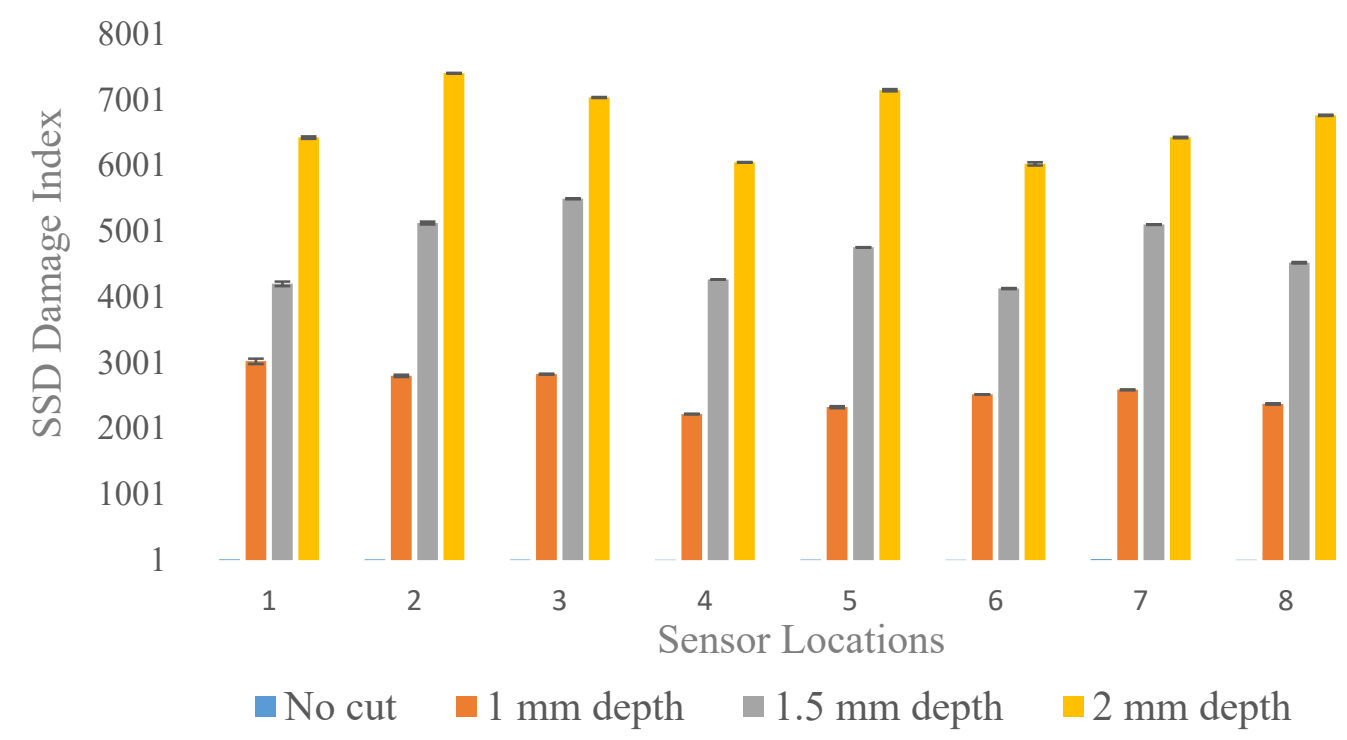

Figure 2.14 SSD damage index values at eight sensor locations before and after the increases in depth of the $100 \mathrm{~mm}$ length longitudinal damage.

\subsection{Summary}

In this study, the SuRE method was implemented for detection of internal and external defects in pipes in contact and noncontact modes. The validity of the method was discussed theoretically. Response of a structure to a fixed excitation remains the same as long as there is no change in the state of the health of the structure. However, the response drastically changes after creation of a damage. A part of change is due to the change in the impedance of the structure and the resultant change in the coupled impedance of the bonded PZT element.

EMIS based methods focus on interpretation of changes due to alteration in the impedance of the attached PZT element. The other part of change is due to the scattering, reflection, and diffraction of waves on their propagation from the exciter to the sensor element. Pitchcatch and pulse-echo based methods more trace effects of these issues on propagating waves in presence of defects. The former is more of a local assessment and the sensitivity 
to defect decreases by getting further from the sensor. The latter is more of a global parameter and can sense changes resulted from defects not necessarily located in close distance from the sensor.

The SSD damage index showed high sensitivity in quantification between different levels of damage. The study showed that the SuRE method can be used to acquire quick and reliable information about the state of the health of pipes. Also, the method, at all sensor locations, showed negligible sensitivity to changes in boundary conditions while it maintained a high sensitivity into defects with different characteristics. Similar to EMIS, the SuRE method is an index based method and calculates the SSD damage index with respect to a baseline response acquired from a pristine part. The SuRE method eliminates the need for EMI analyzers in broad frequency range studies. Spectrum analyzers, simple data acquisition systems and DSPs may be used for data collection and analysis. Therefore, it can safely be considered as a cheaper alternative for EMIS method while it has higher sensitivity to defects that are not in close proximity of the sensor.

The SuRE method uses a broad frequency range for excitation while can use different sensing techniques. The broad frequency range makes the method sensitive to defects with different characteristics, such as size, type and orientation. Due to the fast processing and low cost of instruments, it has a great potential for monitoring of large structures. For instance, it can be used for quick estimations of damage in post-earthquake monitoring of complex pipeline systems. Therefore, it can be effectively used for defect detection and monitoring of large structures. 


\section{CHAPTER 3}

\section{Detection of Defects Using Heterodyning Method}

Early detection of developing structural cracks is extremely important in improving safety and reducing maintenance costs in structural, aerospace, chemical, petrochemical, gas, and oil industries. Nonlinear structural health monitoring methods such as nonlinear wave modulation spectroscopy (NWMS) can detect the cracks in their preliminary stages of development. Such methods usually rely on a combination of a high and a low-frequency excitation for defect detection. However, a priori knowledge of the characteristics of the crack is required for selection of the appropriate frequency combination. In this study, the Comprehensive Heterodyne Effect Based Inspection (CHEBI) method is proposed to address this issue.

The CHEBI method uses ascending and descending broadband frequency sweeps to study the response of the system in wide ranges of frequencies in the time-frequency domain. Therefore, it is not limited to a single combination of the high and the low-frequency components. The proposed method detected cracks from $1 \mathrm{~mm}$ to $25 \mathrm{~mm}$ in length on a dog-bone shaped aluminum specimen. These results confirmed that the CHEBI can be successfully applied for detection of cracks with varying severities without the need for adjustment of the excitation frequencies.

\subsection{Introduction}

Active structural health Monitoring (SHM) methods can be divided into two groups: linear and nonlinear. Different linear and nonlinear methods have been used for crack detection. Most of the initial SHM studies considered the structure to be a linear system. Pitch-catch 
[79]-[84], pulse-echo [1], [85]-[87], electro mechanical impedance spectroscopy (EMIS) [46], [88]-[90] and other methods [75], [76], [91]-[93] mainly look at changes in linear variables. Linear characteristics, such as time of flight between the actuator and the sensor, reflections from the defects, transmitted energy and changes in the impedance of the attached piezoelectric actuator/sensor(s), usually do not change in the early stages of crack nucleation and growth.

Detection of small cracks in their preliminary stages of nucleation and growth may significantly reduce catastrophic failures. Nonlinear methods have gained popularity since they can detect smaller cracks without requiring any information from the pristine structure (baseline data) [51], [53], [55]. Nonlinear methods assume that pristine structures behave like linear systems. In addition, certain types of damages such as debonding, imperfect contacts between mating surfaces, and cracks produce nonlinear effects which can be detected in the preliminary stages of their nucleation and growth [94]-[96]. Time reversal [42], [97], [98] and nonlinear elastic wave spectroscopy (NEWS) methods [99]-[101] like nonlinear resonant ultrasound spectroscopy (NRUS) [102]-[104] and nonlinear wave modulation spectroscopy (NWMS) [105]-[110] (also known as vibro-acoustic modulation) detect the defects such as small fatigue cracks and debondings at very early stages by tracking the development of nonlinear characteristics.

In the vibro-acoustic modulation approach, typically, two different actuators are used to excite the structure with two distinct frequencies, at very low (typically lower than $50 \mathrm{kHz}$ ) and high frequencies (typically a few hundreds of $\mathrm{kHz}$ ). In response to the bitonal excitation, structures with the listed defects respond with newer frequencies in addition to 
the excitation frequencies. These new frequencies are formed at certain frequencies, such as the summation, subtraction, and some other harmonics of the original excitation frequencies. The formation of new frequencies is called heterodyne effect in communication engineering [111]-[113]. Heterodyning effect was introduced by Reginald Fessenden almost a century ago and has been widely used in many engineering applications, such as radio transmitter/receivers, wireless communications, and laser interferometers [114]-[117].

Choosing the appropriate frequency combination(s) play a very important role in successful defect detection using nonlinear methods [6], [118]. For example, Sohn et al.[6] used a combination of a fixed pumping frequency with a chirp signal in the range of $80-110 \mathrm{kHz}$. However, since the pumping frequency was not an appropriate choice, they were not able to see the formation of the side bands in the raw data. Thus, they had to use linear response subtraction (LRS) and synchronous demodulation (SD) for seeing the side bands. Also, they had to repeat their experiments for 21 times using fixed pumping frequencies and a linear chirp probing signal, in search for a better combination of pumping and probing frequencies. In summary, to date, no convenient and systematic approach has been developed for the proper selection of the two initial excitation frequencies.

In this study, we propose the use of two sweep sine waves covering broad ranges of frequencies to address this issue. Use of broad band frequencies eliminates the need for selection of distinctive pumping and probing frequencies. We refer to this new method as Comprehensive Heterodyning Effect Based Inspection (CHEBI). The double broadband sweep sine excitations make the CHEBI method sensitive to defects with different 
characteristics. This eliminates the extensive trial and error for the selection of the low and high frequencies in the inspection of new parts.

The response of the system is studied in the time-frequency domain to evaluate nonlinear characteristics of the system at different excitation frequency combinations. The user may continue to work with the sweep sine wave excitations for extensive study of different kinds of defects at the entire structure or select the best frequency combination for specially specified inspections. In addition to cracks, the proposed method has the potential to detect other types of defects such as debondings and loosely tightened bolts which create similar nonlinear behaviors. The theoretical background, experimental setup, and performance of the proposed SHM system will be discussed in the following sections.

\subsection{Theoretical Background and Numerical Model}

All materials have some level of nonlinearity but the nonlinearity is very small to be detected. Defects such as debonding, delamination, cracks, and loose bolts intensify the nonlinearity in the response of structures to bitonal excitations due to the nonlinear mixing of the excitation frequencies. This nonlinearity leads to the formation of new frequencies at the summation, subtraction and some other harmonics of the excitation frequencies. Such nonlinear behavior can be detected much earlier than any meaningful change in the parameters of the linear methods. The formation of new frequencies can be explained for simple defects such as breathing cracks using equation (3.1) [119], [120]. 


$$
\begin{gathered}
v_{o}=a v_{1} \pm \mathrm{b} v_{2} \pm\left(a v_{1} \pm \mathrm{b} v_{2}\right)^{2} \pm\left(a v_{1} \pm \mathrm{b} v_{2}\right)^{3}+\cdots=\mathrm{a} \sin \left(\omega_{1} t\right) \pm \mathrm{b} \sin \left(\omega_{2} t\right) \pm \\
\mathrm{c}\left(\cos \left(2 \omega_{1} t\right)\right) \pm \mathrm{d}\left(\cos \left(2 \omega_{2} t\right)\right) \pm \mathrm{e}\left(\cos \left(\left(\omega_{1+} \omega_{2}\right) t\right)\right) \pm \mathrm{f}\left(\cos \left(\left(\omega_{1-} \omega_{2}\right) t\right)+\right. \\
g+\cdots
\end{gathered}
$$

Where $\omega 1$ and $\omega 2$ are the angular frequencies of the bitonal excitation. Note that the expected response for an ideal pristine structure can be represented using equation (3.2).

$$
v_{o}=a v_{1} \pm \mathrm{b} v_{2}=\mathrm{a} \sin \left(\omega_{1} t\right) \pm \mathrm{b} \sin \left(\omega_{2} t\right)
$$

It should be emphasized that equations (3.1) and (3.2) only provide a simple mathematical representation for the formation of new frequencies. However, the exact mechanism for the formation of these frequencies in different types of defects is not well understood yet. In this study, before any experimental tests, it was aimed to show the formation of new frequencies in the response of a defected structure to two distinctive single frequencies and also to double broadband excitation, using simple mathematical models. In both models, the dispersion, reflections from boundaries, and attenuation of the surface waves were ignored for the sake of simplification.

For the case of the two distinctive single frequencies, the surface of the plate in Figure 3.1 was excited with a signal which is generated by adding two harmonic signals at the $f_{1}$ and $\mathrm{f}_{2}$ frequencies. These frequencies were selected as $25 \mathrm{kHz}$ and $100 \mathrm{kHz}$, respectively, while many other combinations could be used for the present purpose. Only two of the propagation paths of the dissipating waves between the piezoelectric elements were considered and are shown in the figure. The crack was simulated with a nonlinear system. For simulating the effect of an unknown crack on a propagating wave, a threshold is defined 
that is equal to $1 \%$ of the maximum magnitude of the excitation signal. It is hypothesized that, the propagating waves on one side of the crack will continue to move to the other side if the amplitude is higher than the threshold.

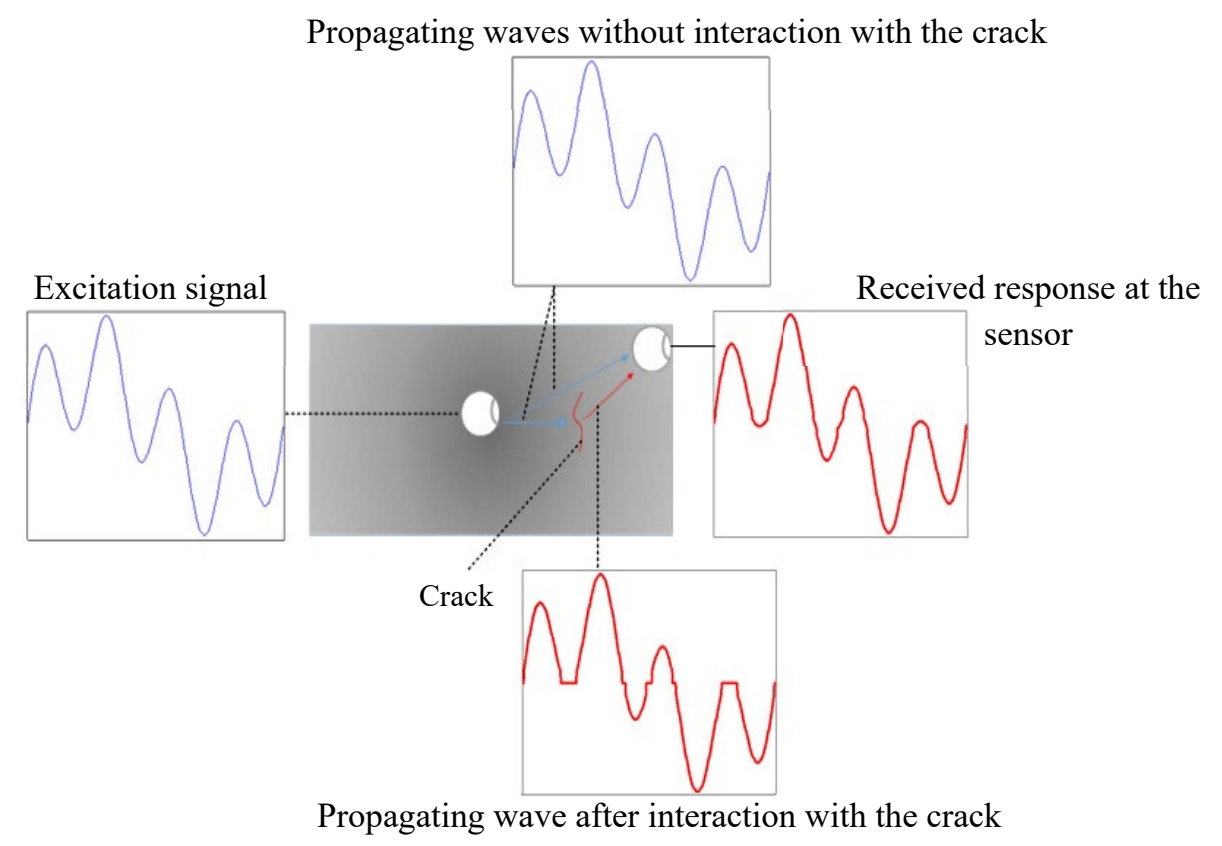

Figure 3.1 Schematic of applied excitations using two distinctive frequencies.

The shape of the signal before and after passing the cracked area is shown in the figure. Some of the propagating waves move around the crack and will not be affected with the separated surfaces of the crack. It is assumed that the piezoelectric element on the other side will receive the superposition of the waves propagated through and around the crack. Based on the assumptions that are made, as it is shown in the figure, the overall shape of the signal received by the second piezoelectric element will be a slightly disturbed form of the excitation signal. 
The Fast Fourier Transform (FFT) of the received response is shown in Figure 3.2. To demonstrate the development of new frequency spikes, the response at the summation and subtraction of the original excitation frequencies are magnified. The development of other new frequency components was also detected which cannot be observed without adequate magnification. The development of new frequencies is a result of the heterodyning effect. Since the excitation signals are very strong and also some of them move around the crack, they have substantially higher spikes compared to the new frequencies. That is, the spikes at the new frequencies can be masked by the powerful spikes of the original excitation frequencies and may not be noticeable without adequate magnification.

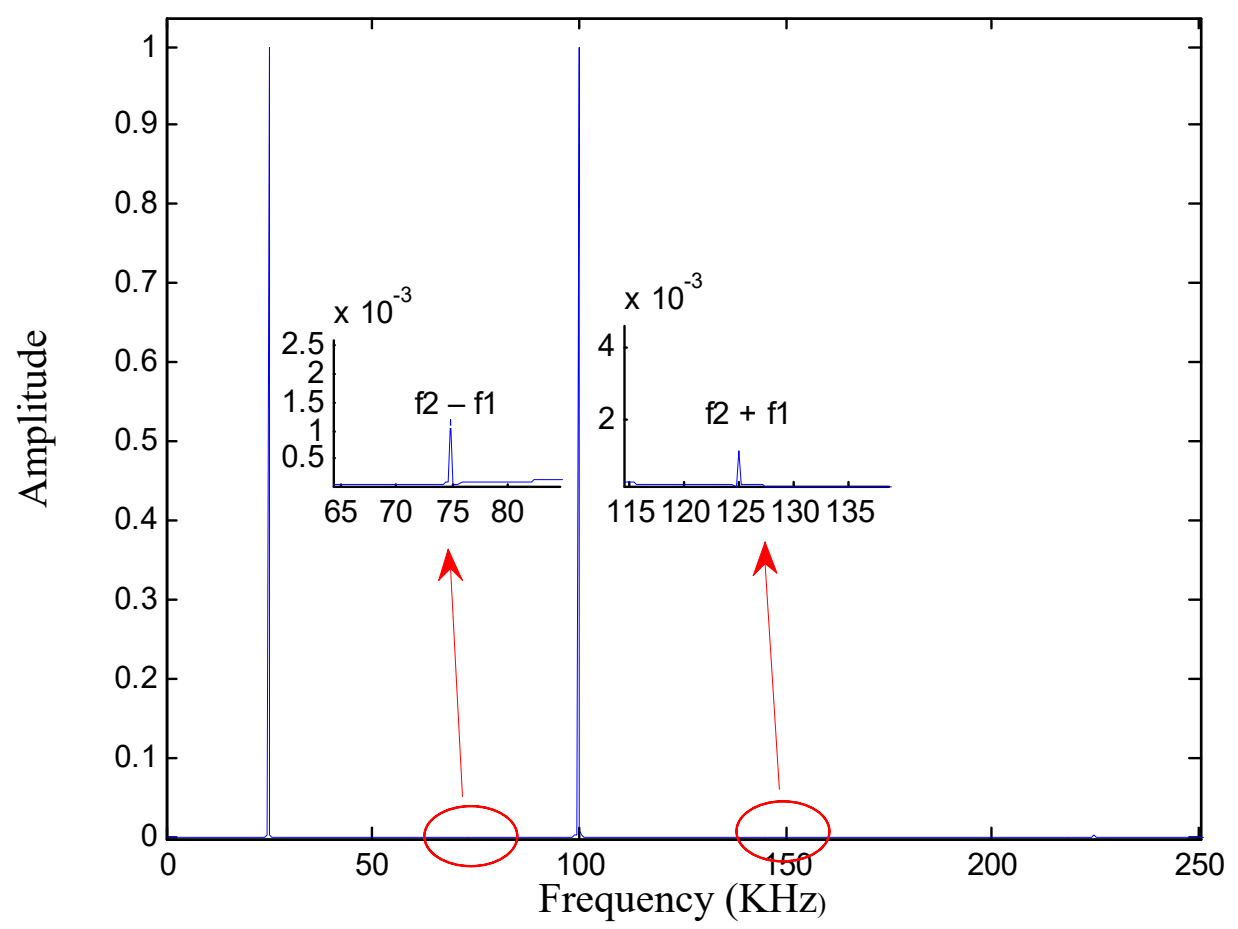

Figure 3.2 Fast Fourier Transform (FFT) of the response signal for the structure with nonlinearity. 
As mentioned earlier, the use of broadband frequency eliminates the need for a priori knowledge about the defect characteristics. A simple numerical model was developed for this purpose. Two sweep signals, $f_{1}$ and $f_{2}$, in the frequency ranges of $200-250 \mathrm{kHz}$ and $350-300 \mathrm{kHz}$ were used as excitation signals. The response of a pristine structure to bitonal sweep excitations was then numerically modeled by calculating Short Time Fourier Transform (STFT) of the response to the excitation and is shown in Figure 3.3 a). As can be seen, $f_{1}$ and $f_{2}$ are the only dominant frequencies in the response.

Then, a nonlinearity was introduced to the response through cutting the amplitudes of $f_{1}$ and $\mathrm{f}_{2}$ signals by $1 \%$, to simulate slight irregularities in the transformation of waves due to an unknown small crack. The development of various new frequencies can be observed in the STFT of the response of the structure with nonlinearity which is shown in Figure 3.3 b). In the mathematical model, among the new frequencies, the $f_{2}-f_{1}$ elements are the most dominant ones after the excitation frequencies. Moreover, in practice, a lower sampling frequency is required for collection of $\mathrm{f}_{2}-\mathrm{f}_{1}$ compared to the higher harmonics. Therefore, in this study, we focused on monitoring of the $\mathrm{f}_{2}-\mathrm{f}_{1}$ elements. It should be noted that length, width, depth, and orientation of a crack can affect its nonlinear behavior and can alter the ratio of the amplitudes of the new frequency spikes versus the ones at the excitation frequencies. 

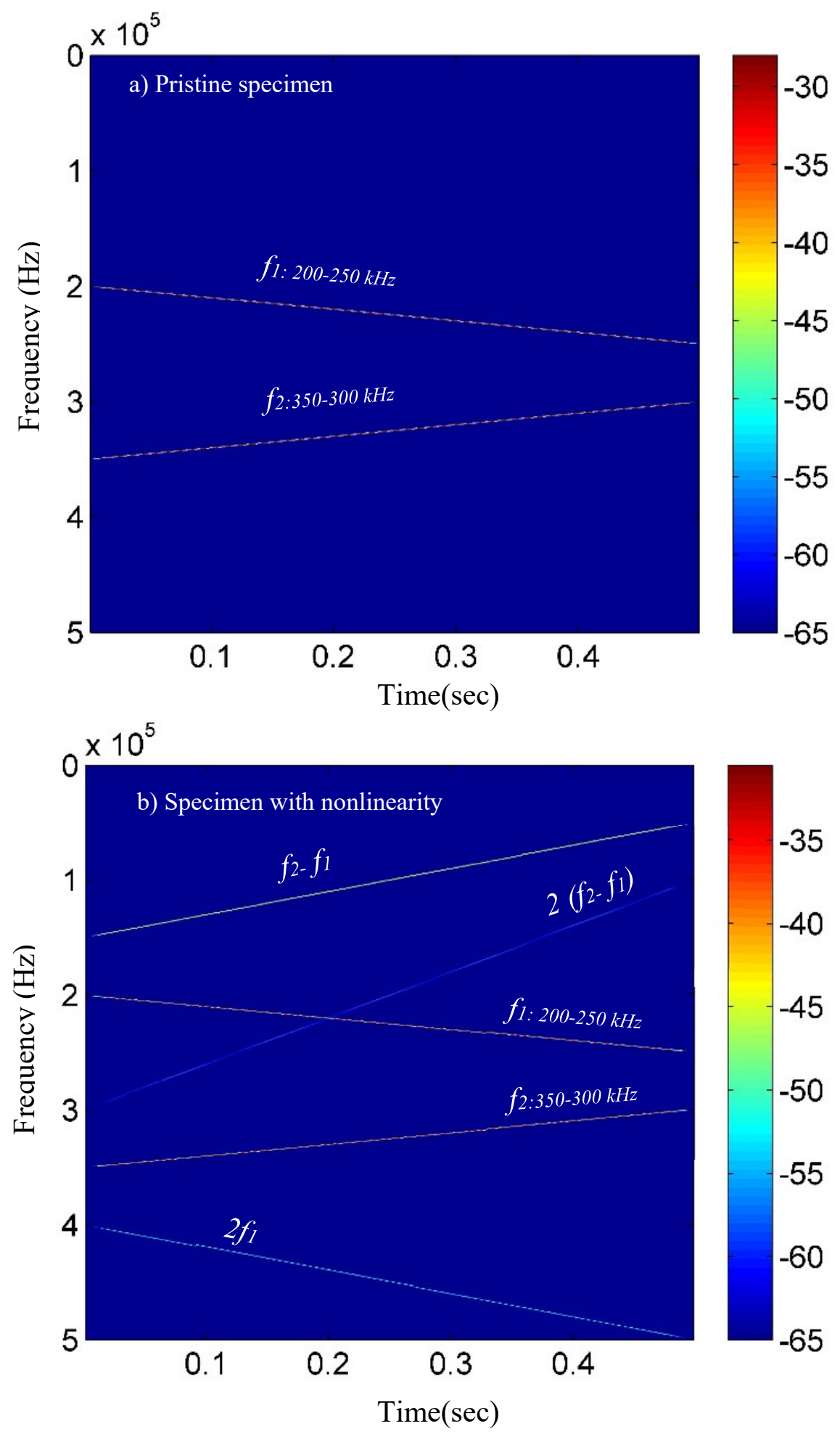

Figure 3.3 The Short Time Fourier Transform (STFT) of the response signals for the pristine and with crack specimens. 


\subsection{Experimental Setup}

The schematic of the experimental set-up and the dimensions are shown in Figure 3.4. Similar aluminum dog bone specimens were prepared for the experimental validation of the CHEBI method. A 5mm notch was cut on the specimen to initiate the crack at the desired location. Three PZT disks with the diameter of $10 \mathrm{~mm}$ and thickness of $2 \mathrm{~mm}$ were bonded to the surface of the specimen at the locations shown in Figure 3.4. Two PZT elements were used as actuators and the third PZT element was used as a sensor. Two Rigol, DG1022A, arbitrary waveform generators (AWG) provided a signal to the actuators. For the implementation of the heterodyning effect, the actuators were excited at different broadband frequencies. The signal of the sensor element was monitored by using the Data Translation DT9832A USB based data acquisition system. The sampling frequency used was $1 \mathrm{MHz}$.

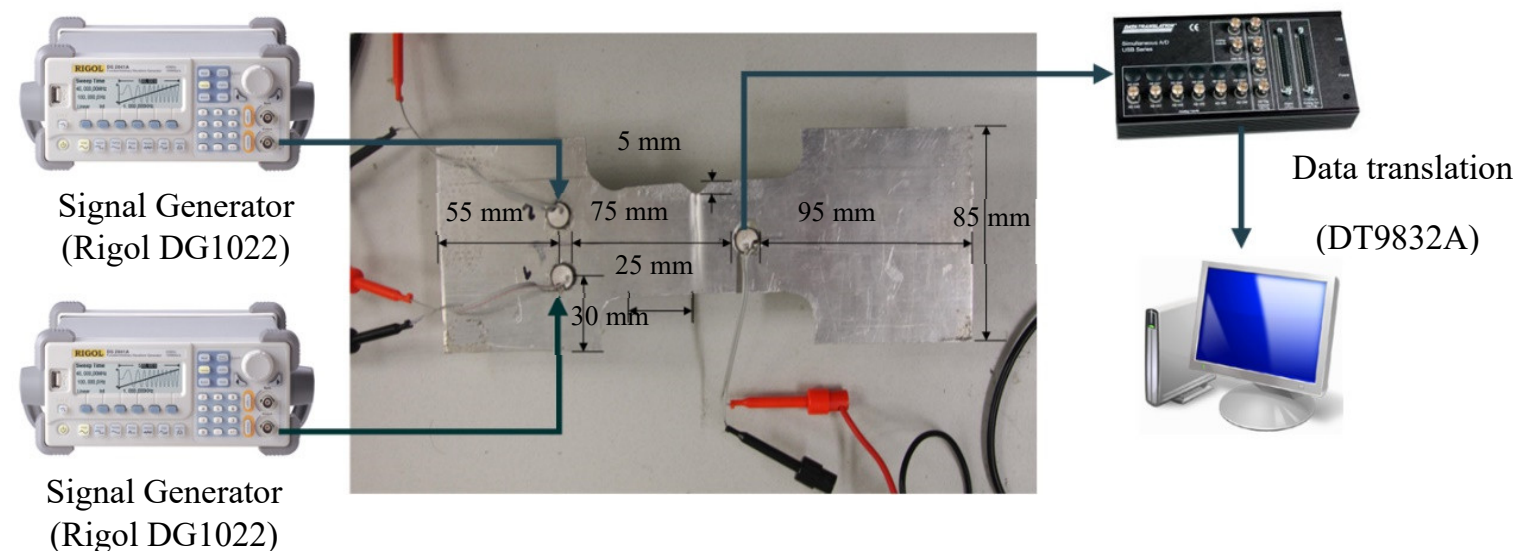

Figure 3.4 Schematic of experimental setup.

The crack was developed by pulling the specimen on the MTS machine in tension. The forces were applied until the crack length reached $25 \mathrm{~mm}$. Note that the aim of this study 
is to detect nonlinear behavior in different crack lengths while using the same double sweep excitations. To accurately investigate the effect of the crack length, the length of the crack was reduced from $25 \mathrm{~mm}$ to $1 \mathrm{~mm}$ in 6 steps by shaving the specimen with a band saw resulting in cracks with the lengths of $19 \mathrm{~mm}, 13 \mathrm{~mm}, 7 \mathrm{~mm}, 3 \mathrm{~mm}$, and $1 \mathrm{~mm}$ respectively. Pictures of the initial crack with $25 \mathrm{~mm}$ and the final one with $1 \mathrm{~mm}$ length were taken by an AmScope MU300 and are shown in Figure 3.5.

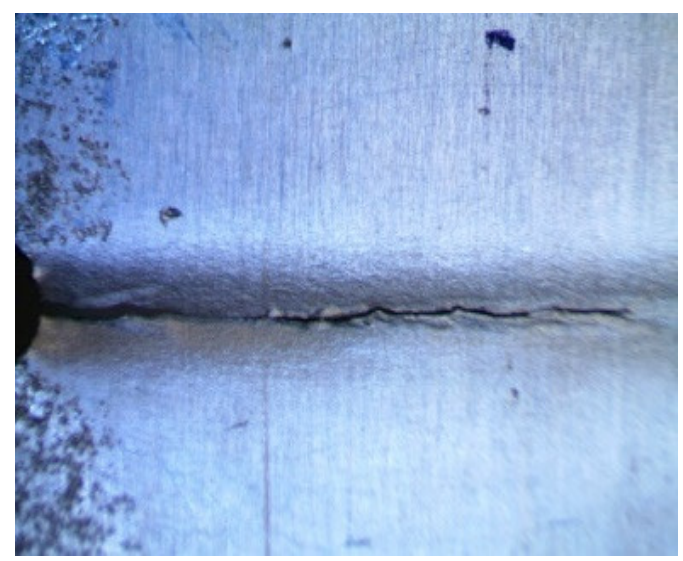

a) The initial crack, $25 \mathrm{~mm}$ in length

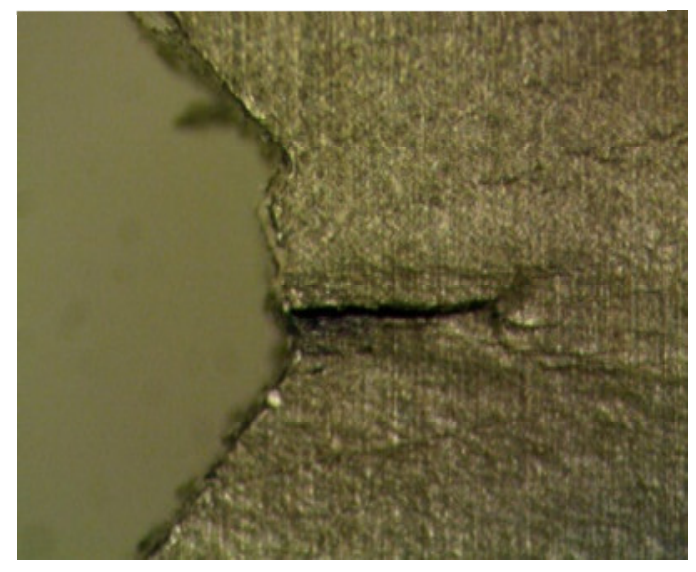

b) Crack shortened to $1 \mathrm{~mm}$ by

Figure 3.5 Pictures of the crack at the beginning (a) and end (b). 


\subsection{Results and Discussions}

In this study, two piezoelectric elements were excited with two separate sweep sine waves.

The amplitudes of the signals were 20 volts peak to peak. The frequencies of the sweep sine waves were in the range of $350-300 \mathrm{kHz}$ (descending) and $200-250 \mathrm{kHz}$ (ascending) resulting in $150-50 \mathrm{kHz}$ monitoring range for the $\mathrm{f}_{2}-\mathrm{f}_{1}$ elements. The response of the sensor was sampled at $1 \mathrm{MHz}$ and evaluated in the time-frequency domain by using the Short Time Fourier Transform (STFT). The Time-Frequency plot of the data for the specimen with the shortest crack length $(1 \mathrm{~mm})$ and the specimen with no crack are shown in Figure 3.6 and Figure 3.7, respectively.

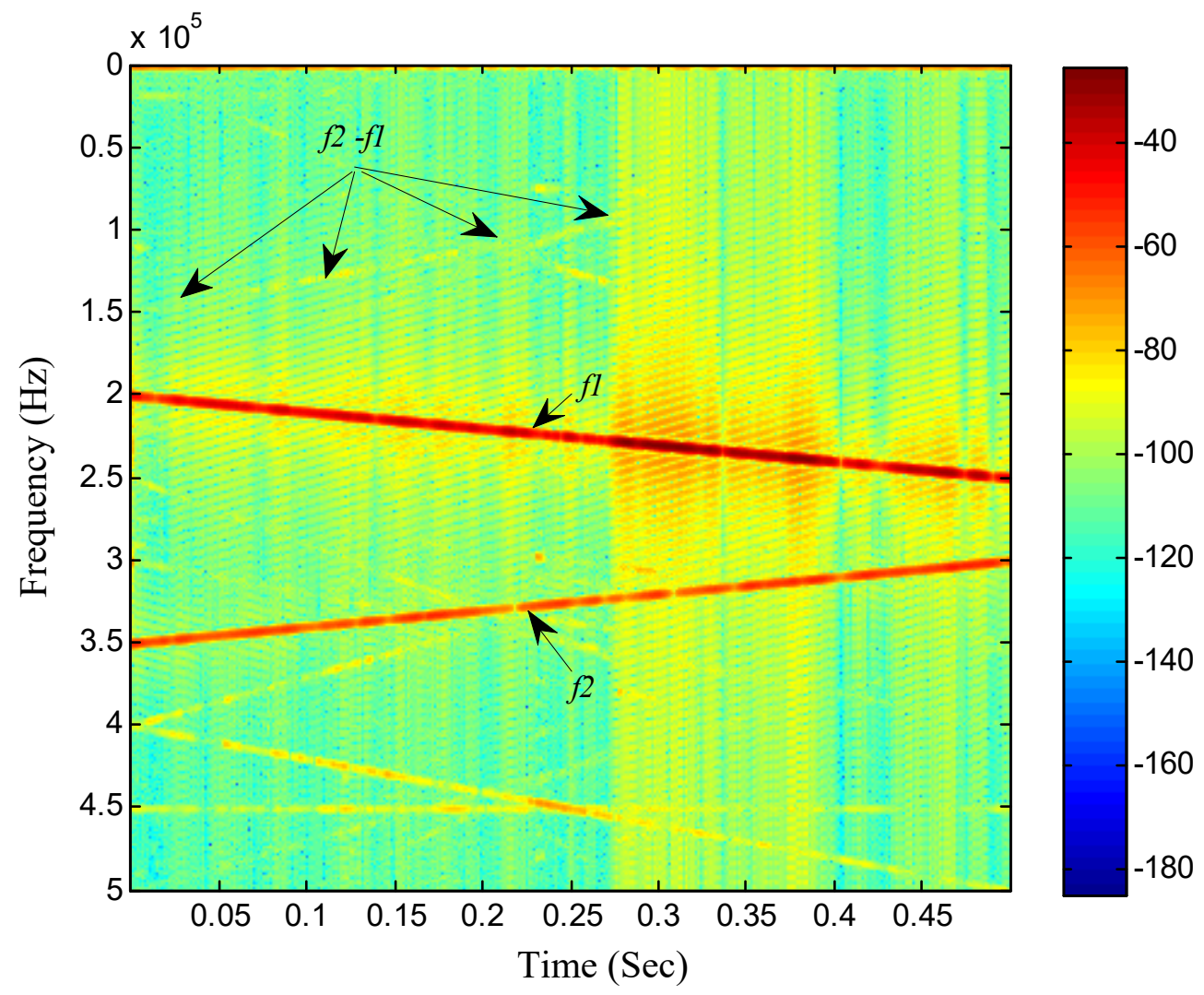

Figure 3.6 The STFT of the response of the structure with $1 \mathrm{~mm}$ long crack to the double sweep excitation. 


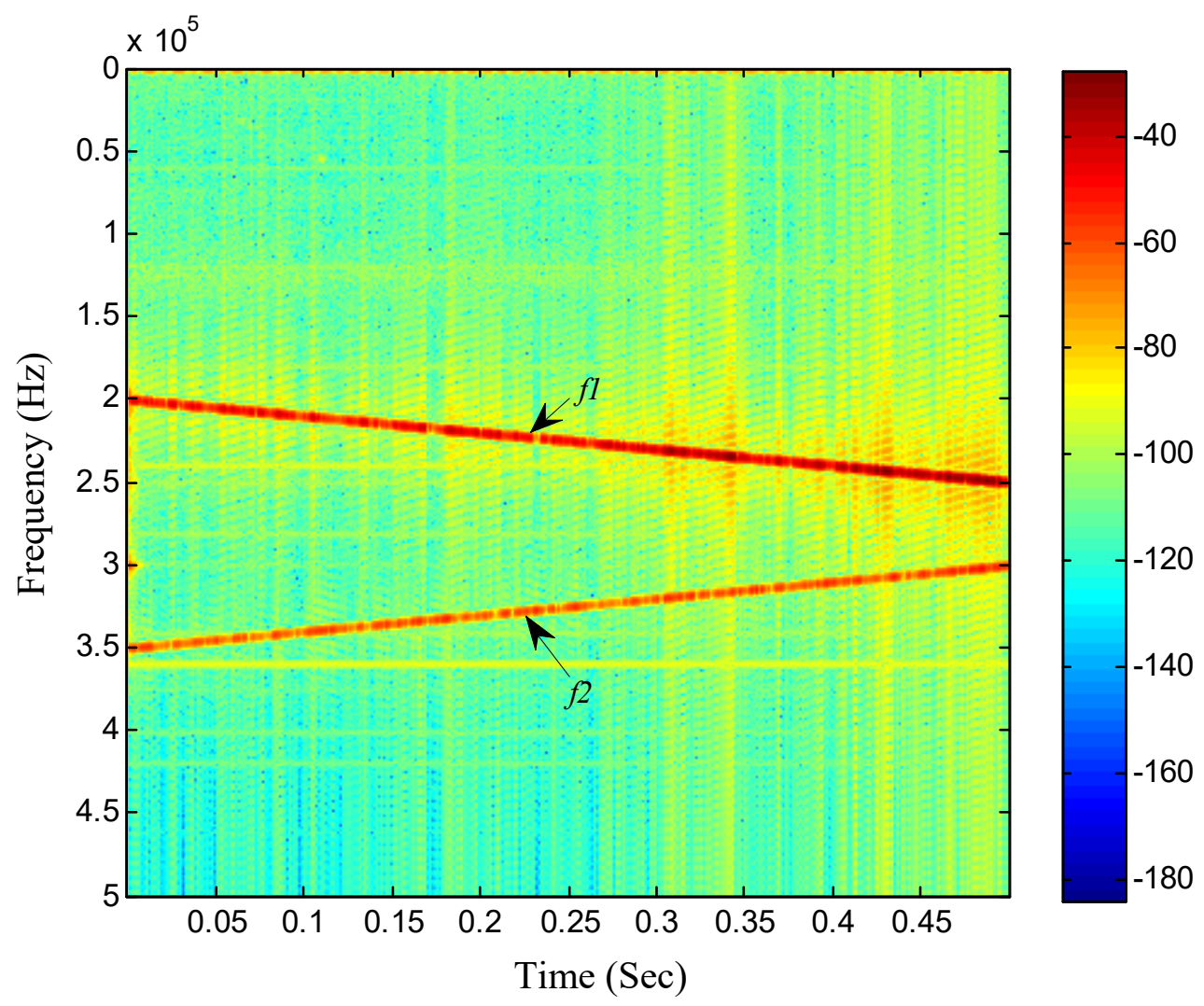

Figure 3.7 The STFT of the response of the pristine specimen to the double sweep excitation.

In both cases, the excitation frequencies were the most dominant components and created boldest responses (red lines). However, in the structure with crack, it can be seen that because of the nonlinear mixing, the structure responds in some newer frequencies along with excitation frequencies. All new frequencies may indicate the presence of damage. However, as explained earlier, in this study the focus is placed upon monitoring the $f_{2}-f_{1}$ elements. By looking at the responses of the specimen with crack and the pristine specimen in Figure 3.6 and Figure 3.7, it can be seen that the frequency components on the $f_{2}-f_{1}$ line are only visible in the structure with $1 \mathrm{~mm}$ long crack. This line started at $150 \mathrm{kHz}$ with $\mathrm{T}=0 \mathrm{sec}$ and ended at $50 \mathrm{kHz}$ with $\mathrm{T}=0.5 \mathrm{sec}$. This line does not exist in the pristine specimen since it is an outcome of the nonlinear mixing of the excitation waves in the crack 
area. Similar to the response in the theoretical study (Figure 3.3), amplitudes of the frequency components on $\mathrm{f}_{2}-\mathrm{f}_{1}$ line are smaller in comparison to the excitation frequencies in the entire frequency range which was swept by the excitation signals.

In addition, the time-frequency plots for 5 different crack lengths: 3, 7, 13, 19, and $25 \mathrm{~mm}$ are shown in Figure 3.8 - Figure 3.12 where the same double sweep excitation used for all of them. The $\mathrm{f}_{2}-\mathrm{f}_{1}$ line exists in all the plots and demonstrates the comprehensiveness and reliability of the CHEBI method in detection of different lengths of crack without any need for alteration of the excitation signals. As expected, not all the excitation frequency combinations work well and show the appropriate strength.

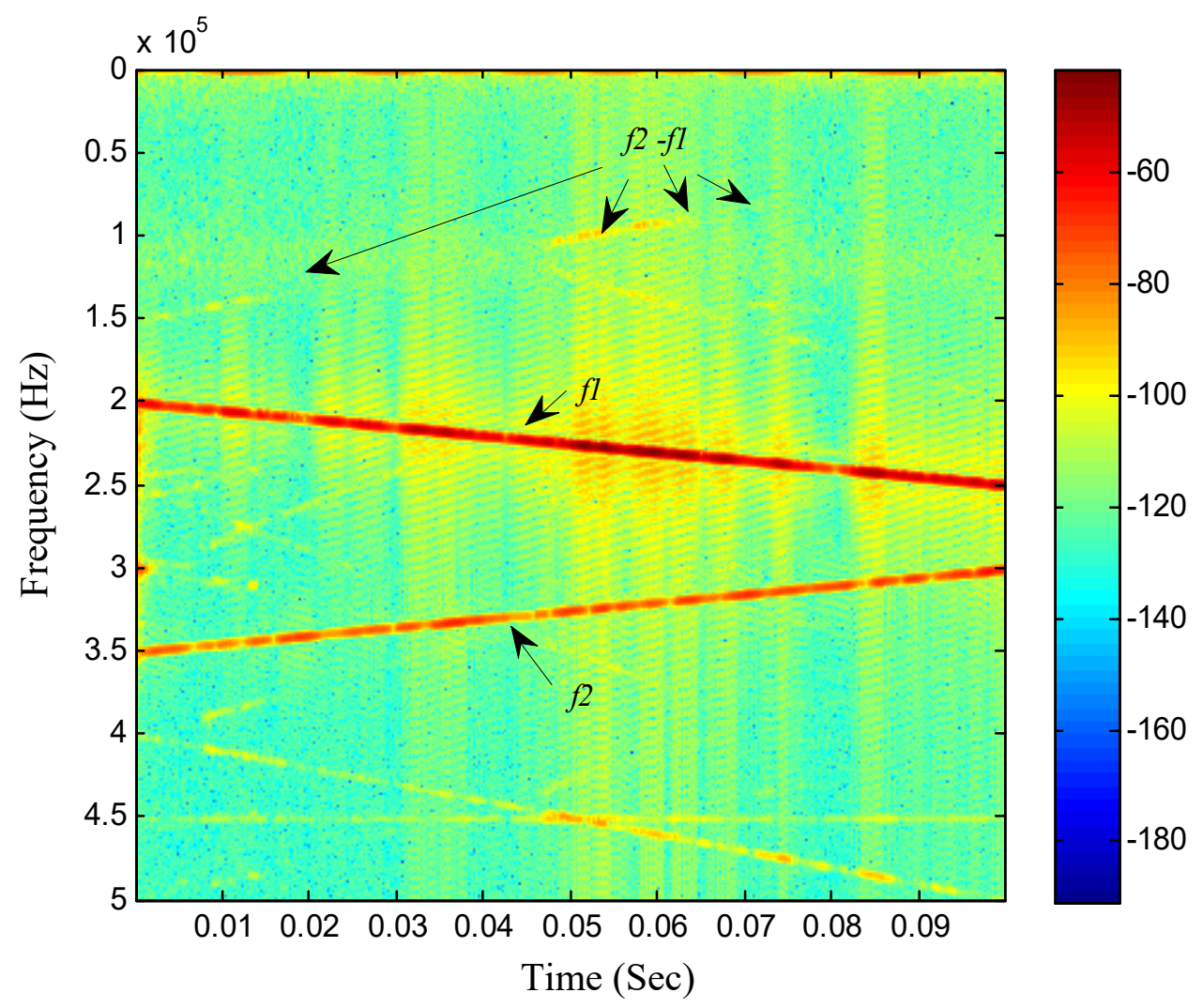

Figure 3.8 The STFT of the response of the structure with $25 \mathrm{~mm}$ long crack to the double sweep excitation. 


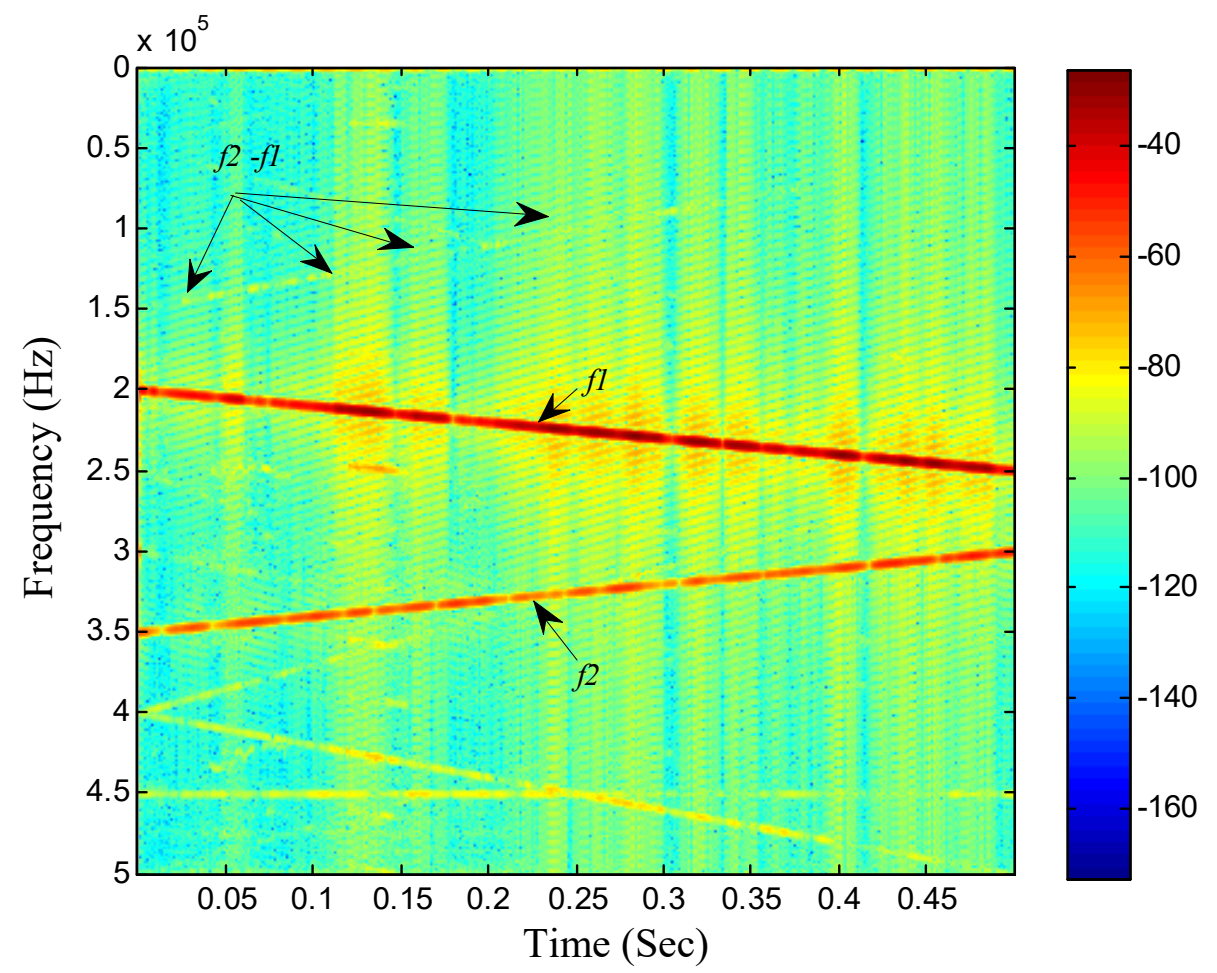

Figure 3.9 The STFT of the response of the structure with $19 \mathrm{~mm}$ long crack to the double sweep excitation.

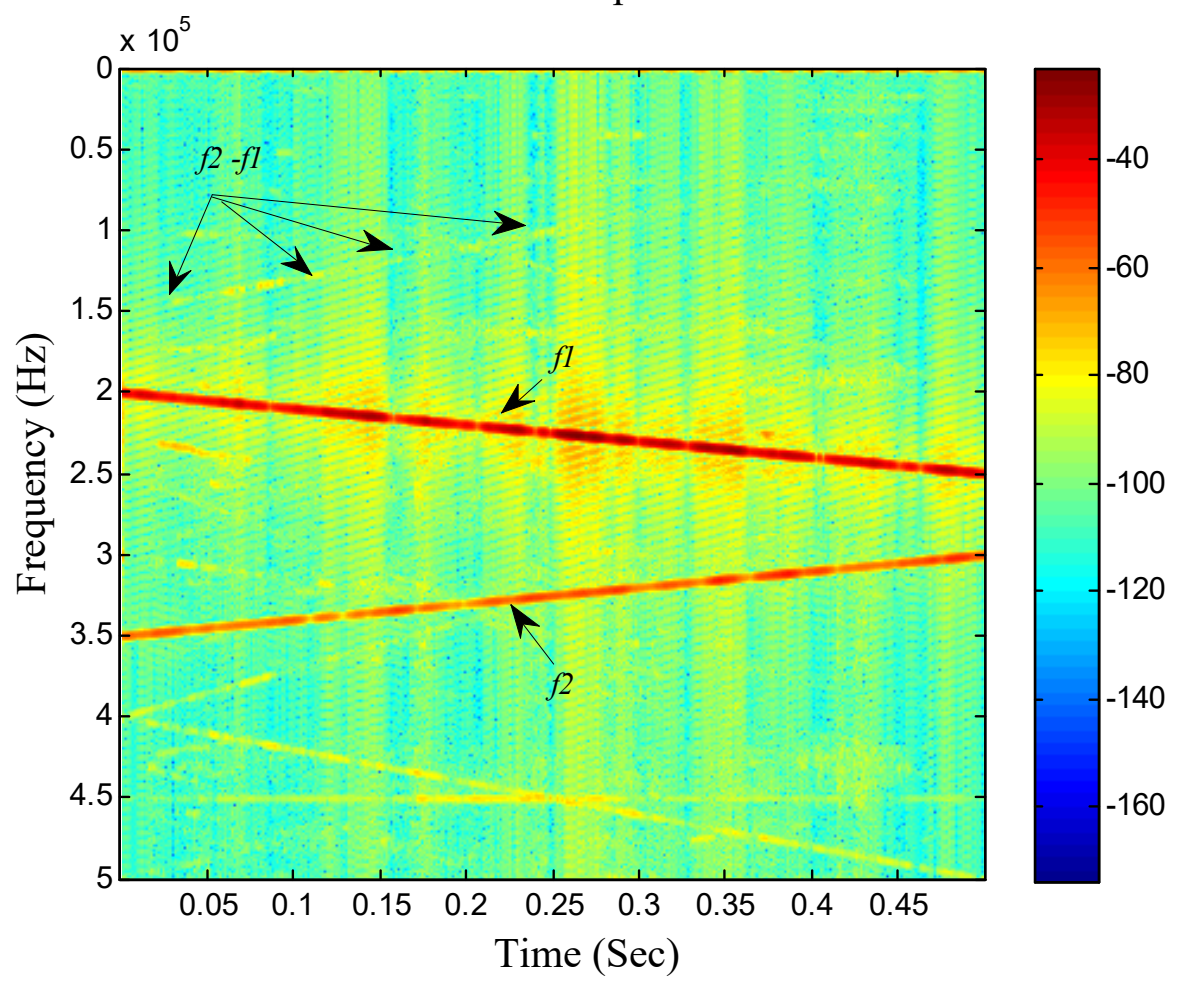

Figure 3.10 The STFT of the response of the structure with $13 \mathrm{~mm}$ long crack to the double sweep excitation. 


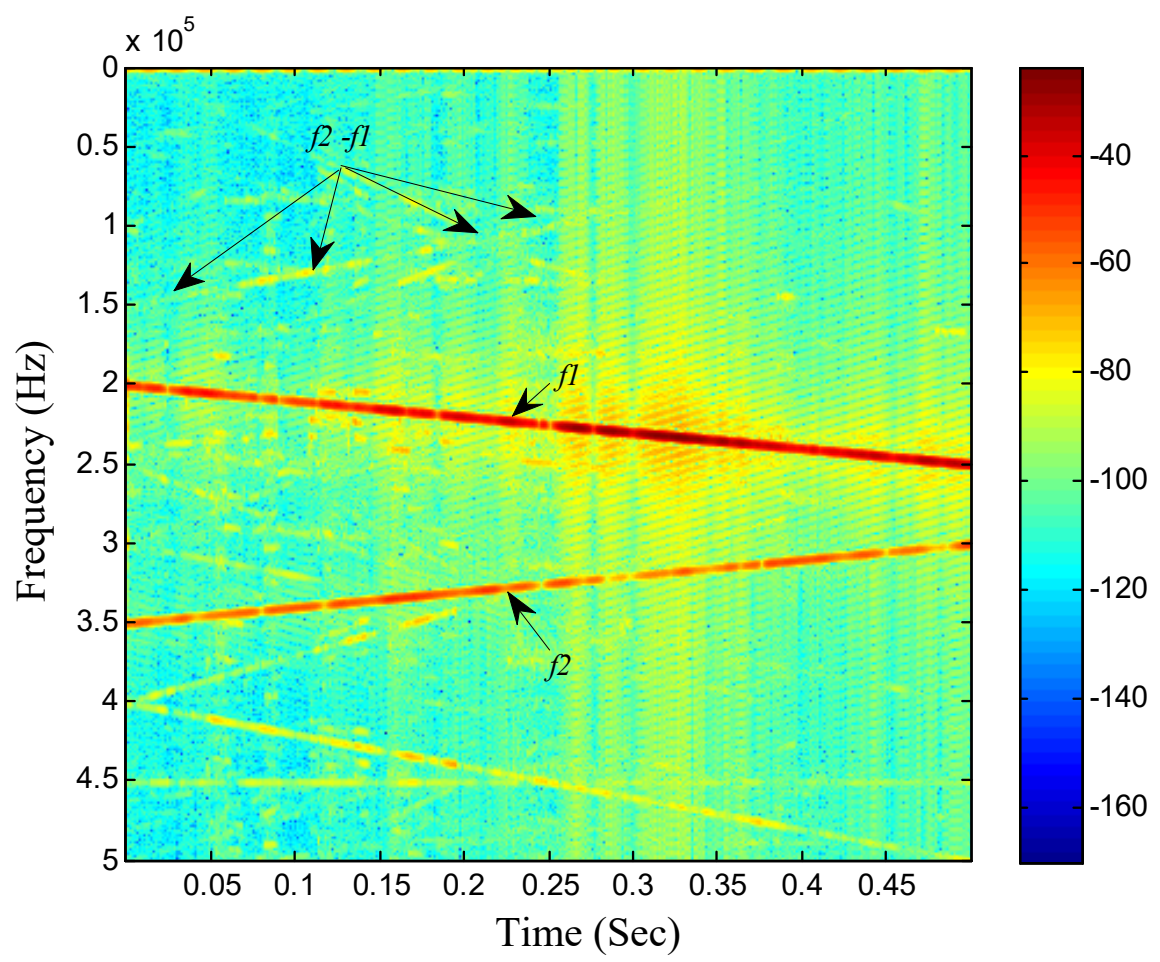

Figure 3.11 The STFT of the response of the structure with $7 \mathrm{~mm}$ long crack to the

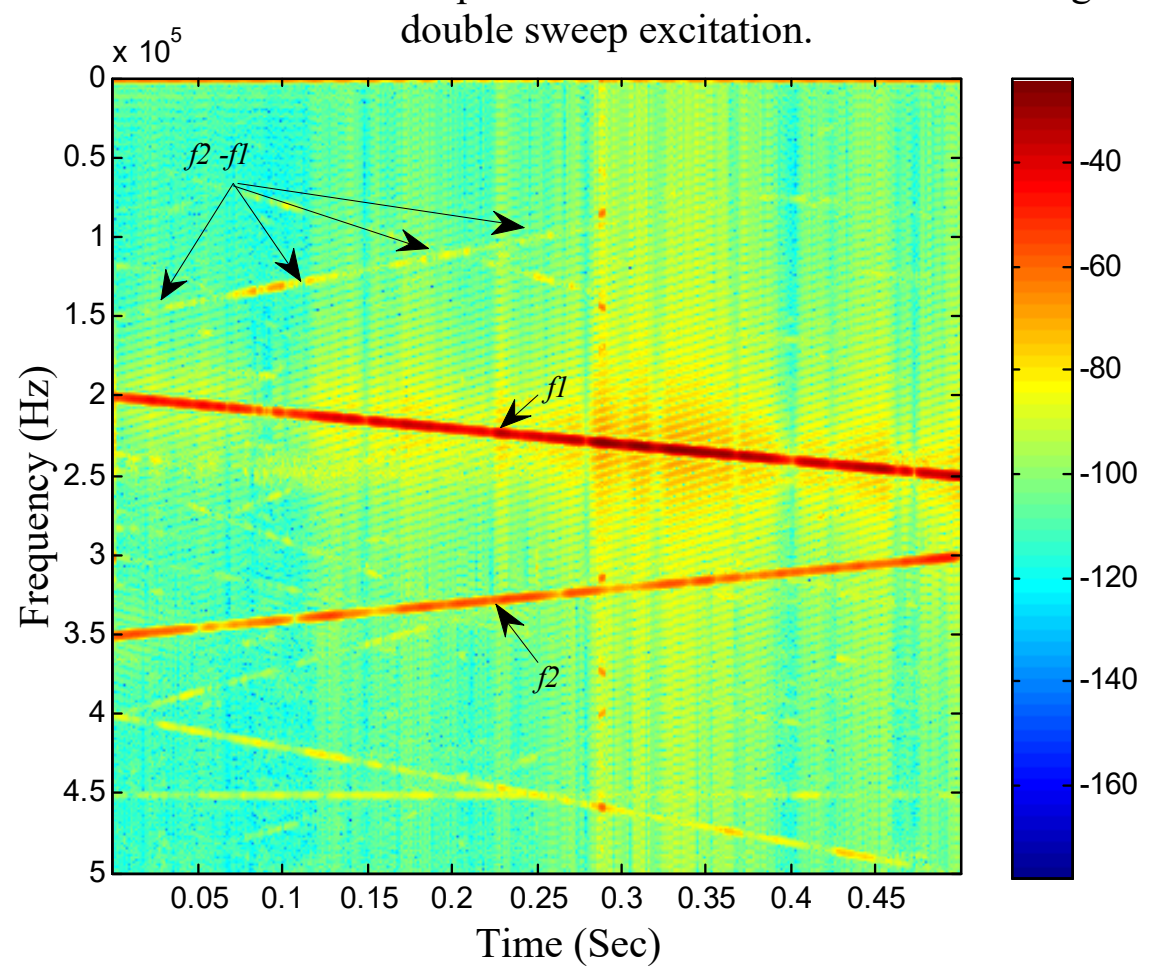

Figure 3.12 The STFT of the response of the structure with $3 \mathrm{~mm}$ long crack to the double sweep excitation. 
Amplitudes of nonlinear response elements on different points of $f_{2}-f_{1}$ line vary depending on the corresponding frequency combination. The Amplitudes of the frequency components of five points located on $\mathrm{f}_{2}-\mathrm{f}_{1}$ line which appear the weakest in Figure 3.6 and equivalent amplitudes of those components from Figure 3.7 were tabulated in Table 3.1. Generally, the amplitudes of the frequency components on the subtraction line $\left(f_{2}-f_{1}\right)$ in the pristine specimen are in the order of the amplitudes of the noise. The difference column clearly shows that the amplitudes of the frequency components of these points on the $f_{2}-f_{1}$ line were stronger than the healthy sample without any cracks. However, by using the CHEBI method the best frequency combination can be easily chosen from the STFT of the response. Therefore, using the double sweep excitation eliminates the need for finding the right combination(s) of excitation frequencies and yields more reliable results.

Table 3.1 Amplitudes of 5 frequency components on $\mathrm{f}_{2}-\mathrm{f}_{1}$ line.

\begin{tabular}{|c|c|c|c|c|}
\hline \multirow{2}{*}{$\begin{array}{c}\text { Time } \\
(\mathrm{sec})\end{array}$} & $\begin{array}{c}\left(\mathrm{f}_{2}-\mathrm{f}_{1}\right) \\
\text { Frequency } \\
(\mathrm{kHz})\end{array}$ & Perfect & With crack & $\begin{array}{c}\text { Absolute } \\
\text { Difference }\end{array}$ \\
\cline { 3 - 5 } & 147.60 & -113.6 & -98.53 & 15.07 \\
\hline 0.012 & 130.00 & -119.9 & -84.69 & 35.21 \\
\hline 0.100 & 100.00 & -112.9 & -92.97 & 19.93 \\
\hline 0.25 & 80.00 & -117.7 & -99.61 & 18.09 \\
\hline 0.35 & 60.00 & -107.9 & -99.98 & 7.92 \\
\hline 0.45 & \multicolumn{3}{|c}{} \\
\hline
\end{tabular}

In the next step, to show the efficiency of the CHEBI method in detection of other defects, we applied it for detection of a loose bolt in a washer system and two plates held together with four nuts and bolts. The schematics of the tests are shown in Figure 3.13. 
a)

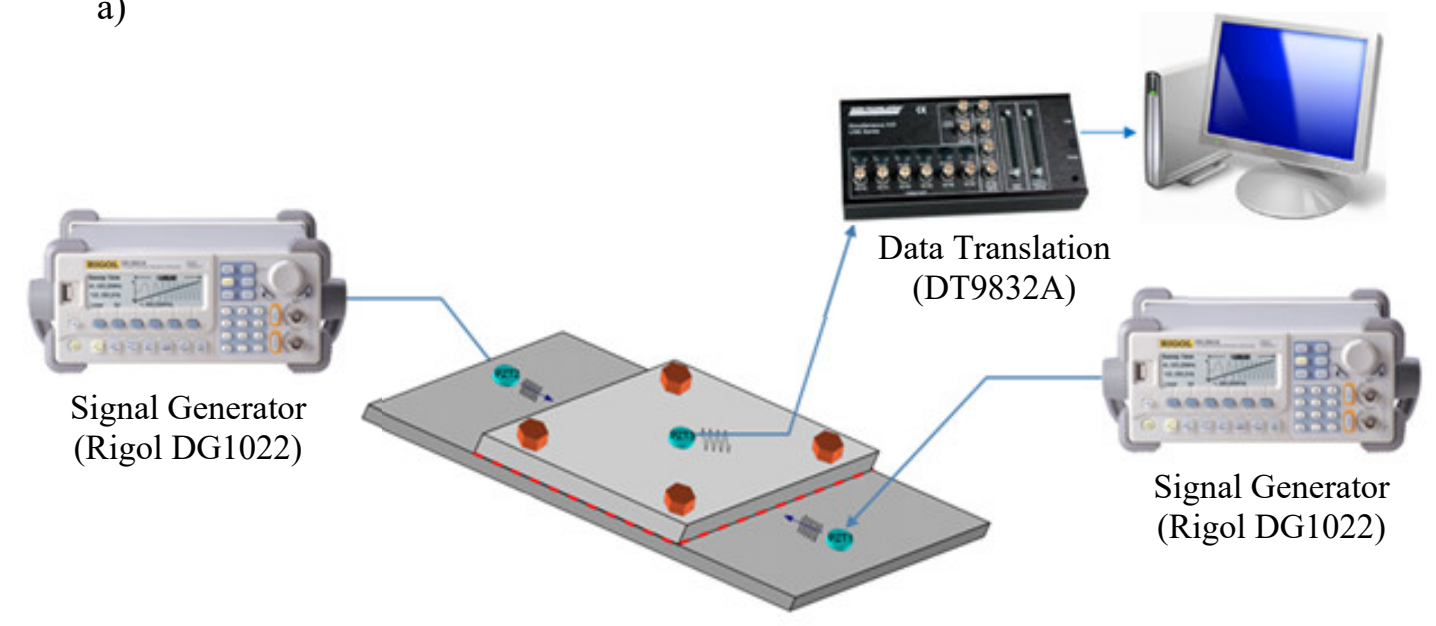

b)

Signal Generator

(Rigol DG1022)
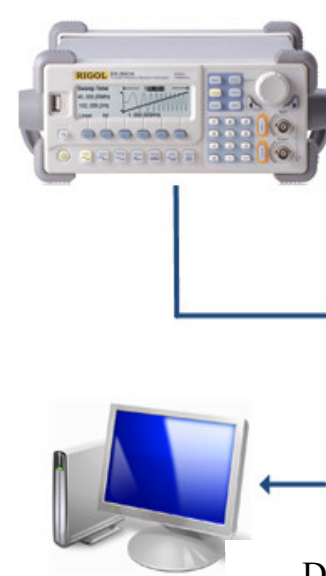
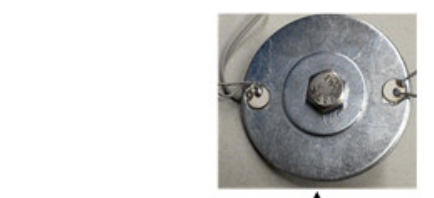

Signal Generator (Rigol DG1022)
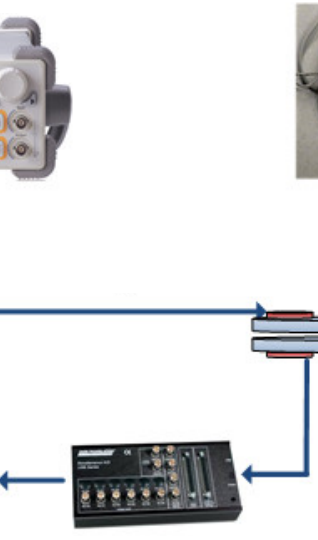

Data Translation

(DT9832A)

Figure 3.13 Tests for detection of loose bolt using a combination of broad band frequency and single frequency a) plates system $b$ ) washer system.

Although, in the original form of CHEBI, we use two sweep sine waves but to show the flexibility of the CHEBI method, this time $f_{1}$ was selected as a sweep sine wave in the range of $30-100 \mathrm{kHz}$ and $\mathrm{f}_{2}$ was selected to be equal to $100 \mathrm{kHz}$. The STFTs of the responses in loose condition and after tightening by $2 \mathrm{Nm}$ for the plate and washer systems are shown in Figure 3.14 and Figure 3.15, respectively. As can be seen from both figures 
in the loose condition and because of the light contact there is a strong signal at $f_{2}-f_{1}$ line. However, when the bolts are tightened by $2 \mathrm{Nm}$ the nonlinear effect decreases substantially. The nonlinear effect doesn't completely disappear due to the slight nonlinearity of mating surfaces at all times. But, by defining a threshold a loose bolt can be easily identified from a well tightened bolt.
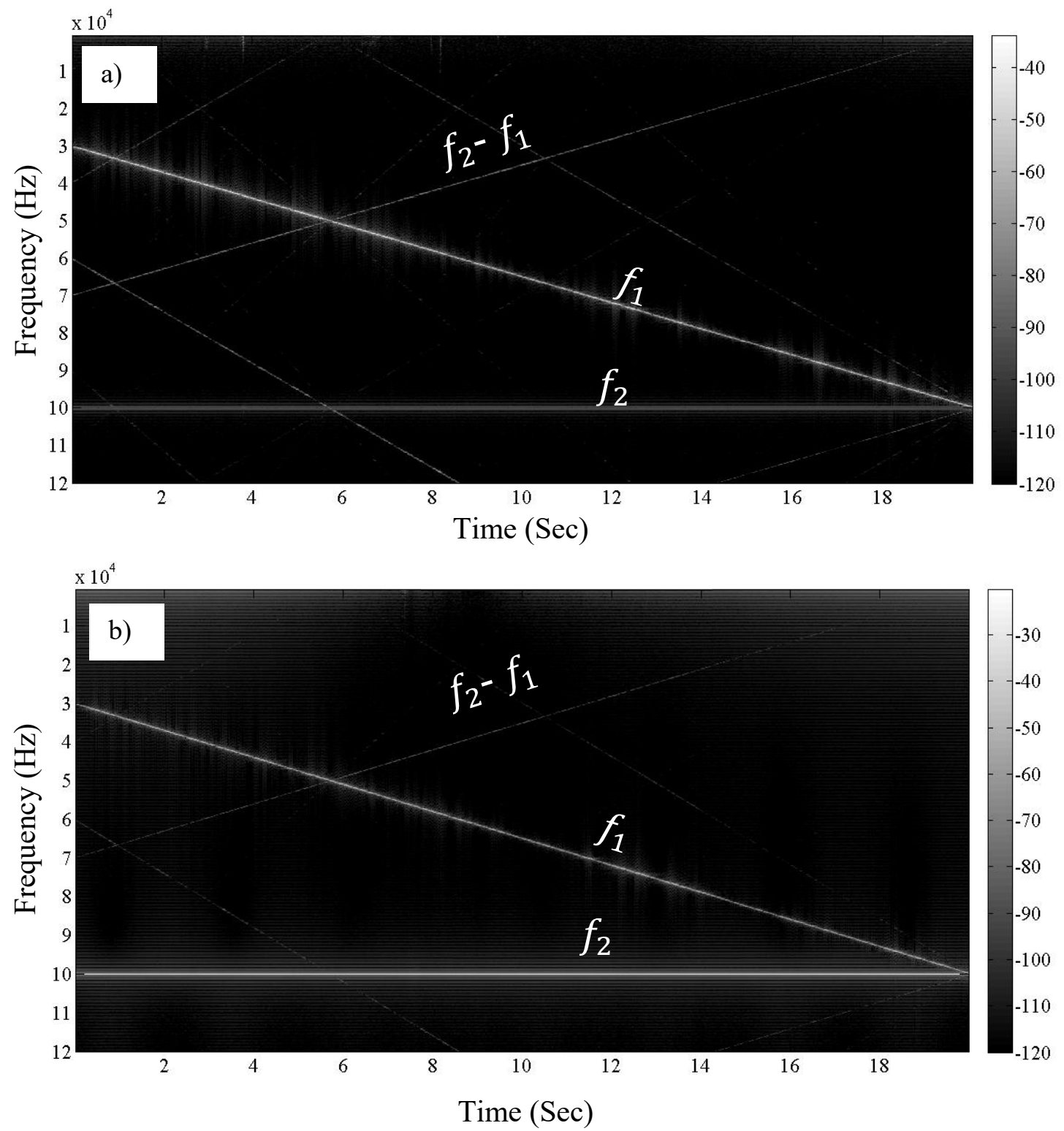

Figure 3.14 Response of the plate to double excitation a) loose bolt b) tightened by $2 \mathrm{Nm}$. 

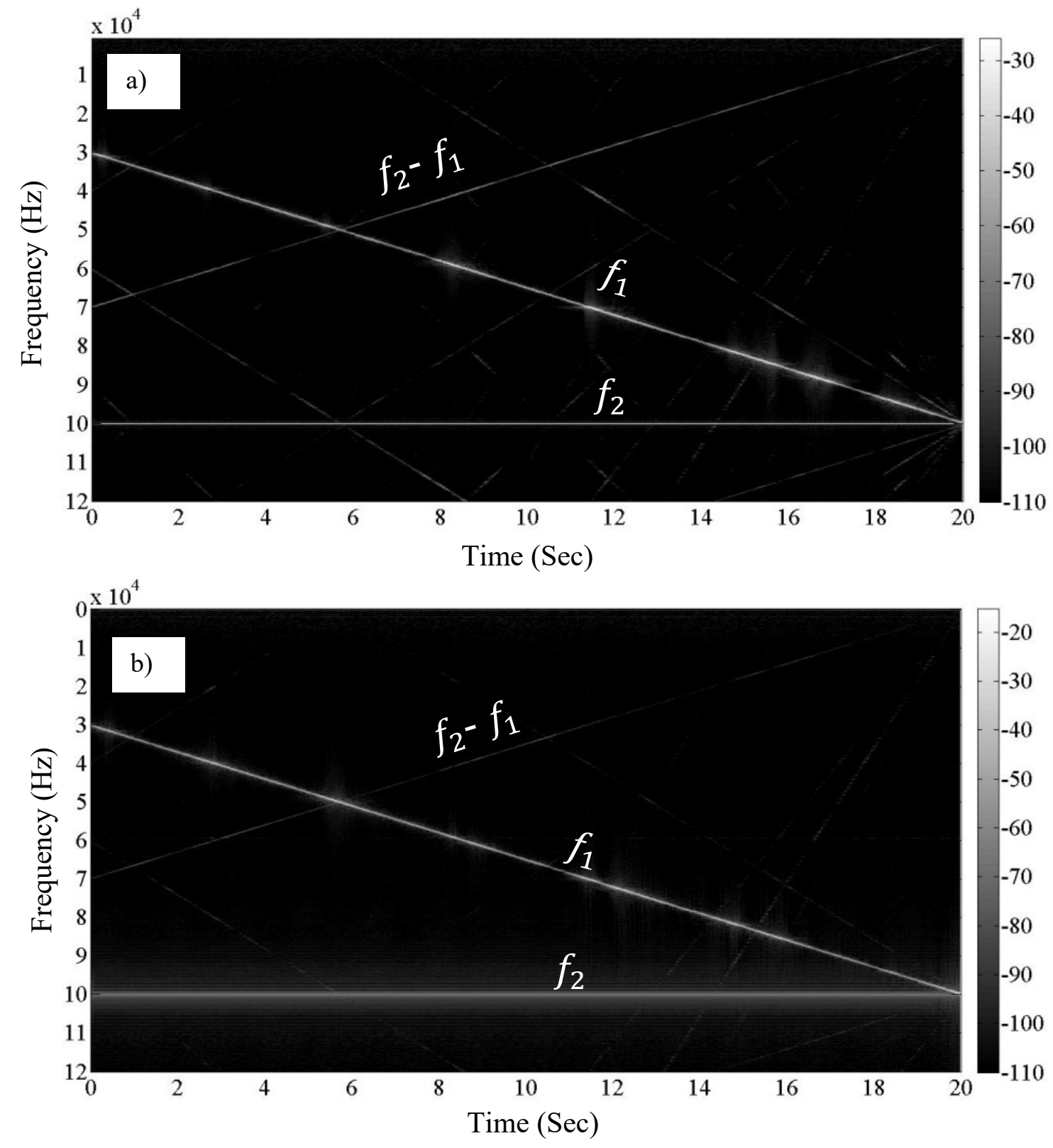

Figure 3.15 Response of the washer system to double excitation a) loose bolt b) tightened by $2 \mathrm{Nm}$.

\subsection{Summary}

Recent studies have shown that combinations of very low and high harmonic excitations may be used to detect defects in initial stages of their development. A healthy structure primarily responds in excitation frequencies. However, defects such as cracks, debonding, 
delamination and loose bolts cause the structure to respond with new frequencies. Successful detection of different types of defects requires careful selection of excitation frequencies. In order to identify the appropriate excitation frequency, however, the majority of such methods require a priori knowledge of the characteristics of the defect under consideration. This makes the whole enterprise of detecting structural defects logically circular as there is usually limited a priori information about the characteristics of defects that are yet to be detected.

In this study, a comprehensive heterodyne effect based inspection method was implemented by considering that the structure with defect responds to the excitation with two high frequency components like a nonlinear mixer and new frequencies are created. Unlike previously available methods, using the CHEBI method, it is possible to analyze the nonlinear behavior of a structure in multiple and higher ranges of frequency combinations in one single test. The method doesn't require one of the frequencies to be very low. Additionally, application of broad frequency ranges with ascending and descending sweep excitations allows the method to be sensitive to many various types of potential defects. This new approach may be easily applied for detection of different types of defects without significant modifications.

In this study, initially, a numerical model was used to show the formation of new frequencies in the response of a structure with nonlinearity to double sweep excitations. In the experimental section, a tension specimen was prepared and different lengths of cracks were examined. Two piezoelectric elements excited the specimen with sweep sine waves while another monitored the response of the specimen. The frequency content of the 
monitored signal was the same as the excitation signal before the crack was introduced. However, for crack lengths ranging from $1 \mathrm{~mm}$ to $25 \mathrm{~mm}$ the specimen responded with the expected new frequency components. That is, the $\left(f_{2}-f_{1}\right)$ line can be clearly observed in all STFT plots of the specimens with a crack.

Different modes and excitation frequencies do not show similar interactions with the existing defects of a structure. Therefore, the broad frequency range sweep excitations make the CHEBI method sensitive to a broader range of potential defects. The results showed that the double sweep sine wave excitations can be effectively used for detection of cracks of varying lengths. The CHEBI method eliminates the need for selection of appropriate or practical combinations of single frequencies for the fixed bitonal excitations and reduces the number of tests that are required for ensuring successful inspection of a structure. The method may be used in different industries for quick and reliable assessment of structures without requiring baseline data. 


\section{CHAPTER 4}

\section{Sensor-less Monitoring of Structures}

To date, sensors have been the inevitable part of structural health monitoring (SHM) systems. SHM methods identify damage by extracting useful information from data collected by sensors and through implementing appropriate data processing algorithms. However, sensors themselves are prone to damage and are limited to certain conditions. Typically, SHM systems are implemented in costly critical structures because of the excessive cost of sensors, data acquisition, signal processing and required expertise for the analysis of the information. This section proposes the first sensor-free active monitoring technique in the field of SHM. The goal is to create sound or verbal warnings at the location of defects without any sensor, processor, or speaker. The feasibility of the concept was demonstrated by detecting loose bolts in a bolted plate system. Also, to demonstrate the potential of this system in industrial applications, the concept was implemented in a typical washer system. Additionally, the characteristics of the audible alarms were studied at different torque levels. The test structures were excited with two distinct and carefully designed signals which led to creation of audible alarms at the location of loose bolts. The proposed concept may be used as a low cost SHM system or implemented as a backup for conventional SHM systems.

\subsection{Introduction}

As it was discussed in the previous section, monitoring the transformation of a structure from the linear state into the nonlinear state in presence of defects has been used for development of nonlinear SHM methods. In these approaches, the dependency to the baseline or reference data is reduced or eliminated. The Time reversal method [42], [97], 
[121], nonlinear elastic wave spectroscopy approaches [101], [122]-[125] and its variations, such as nonlinear resonant ultrasound spectroscopy (NRUS) [126]-[128], and nonlinear wave modulation spectroscopy (NWMS) or other similar methods, such as impact modulation [105], [110], [118], [129]-[131] are some examples of the nonlinear approaches in the literature. In addition to eliminating the need for baseline information, the nonlinear methods can detect smaller defects with much higher sensitivity compared to the linear methods.

In the NWMS approach and other similar methods, the surface of the structure is excited with two distinct frequencies. A pristine structure primarily responds in the excitation frequencies. However, defects, such as debonding, imperfect contacts between mating surfaces, and cracks produce nonlinear effects in the response of the structure. These nonlinearities manifest themselves through the formation of newer frequencies, different than the excitation frequencies, in the response of the structure to the bitonal excitation. These new frequencies can be observed at the summation, subtraction (side bands) and some other harmonics of the excitation frequencies even at the preliminary stages of development of defects. Formation of new frequencies in the output resulting from the two distinct frequencies applied to a nonlinear system is known as the heterodyning effect in communication engineering and has been used in many engineering applications such as radio transmitters, receivers, and laser interferometers [111], [113]-[117].

In conventional nonlinear NDE and SHM methods like NWMS, the formation of side bands in the response of the structures to the bitonal excitation is monitored by using sensors. It should be noted that sensors must be compatible with the performance of the 
structure in potentially rugged environments and should be capable of inspecting rather large areas of the host structure without being displaced. To date, there is no ideal sensor which can be used in all engineering applications and environments. Also, a sensor which holds all characteristics of an ideal sensor would likely be expensive and would be difficult to use in high quantities for monitoring large structures. Moreover, processors, data acquisition devices, and skilled operators are required for interpretation of data collected by sensors. These issues and complexities in the collection and interpretation of data from in-service structures make them vulnerable to error [132] and limit their implementation into critical structures.

It should be noted that humans, theoretically, hear sounds in the frequency range of $20 \mathrm{~Hz}$ - $20 \mathrm{kHz}$. Therefore, if we keep the difference between the excitation frequencies below 20 $\mathrm{kHz}$, then the smaller sideband, $\mathrm{f}_{2}-\mathrm{f}_{1}$, in the response of structures with defects would be in the audible range. To date, no research has focused on the development of sensor-free SHM (SSHM) methods using this phenomenon.

The objective of this section is to propose a new method which doesn't need sensor(s), data acquisition device(s), processor(s) and well-trained operators by implementing the heterodyne effect for detection of loose bolts. The implementation of the proposed lowcost system is feasible for many consumer products. The SSHM only uses two actuators and triggers the audible alarm at the location of the loose bolts. The concept was tested on two plates which were held together with four nuts and bolts. The concept was also validated on the most widely used industrial fastener components: washers. A washer system was designed for implementation of the proposed approach and successfully 
demonstrated the potential of the SSHM concept. The theoretical background, experimental setup, and performance of the proposed SSHM systems are discussed in the following sections.

\subsection{Theoretical Background}

All materials have some level of nonlinearity, but a solid structure with well tightened bolts mainly behaves like a linear system [106]. In the frequency response of these structures to bitonal excitations, the spectra are typically at the excitation frequencies. However, microcracks, delamination, debonding and mating surfaces distributed in a structure can act as nonlinear mixers for excitation waves. These types of damage create additional harmonic waves with the newer frequencies, as a result of the distortion or partial transmission of the waves. The amplitudes of the created waves depend on the location and type of defects.

The expected response of an ideal structure, without any nonlinearity, to a bitonal excitation consisting of $v_{1}=\sin \left(\omega_{1} t\right)$ and $v_{2}=\sin \left(\omega_{2} t\right)$ can be explained by equation (3.1). The formation of new frequencies in the response of a structure with sources of nonlinearity, defects, can be illustrated by equation (3.2).

In a bolted structure, if the surfaces are not properly mated and there is a small gap between them, then the light contact will serve as a nonlinear medium for propagation of waves. From equation (3.2) it can be shown that the response of such a system to the bitonal excitation consists of the excitation frequencies $\omega_{1}$ and $\omega_{2}$, and other frequencies including summation and subtraction of these frequencies. 
Therefore, by choosing the excitation frequencies in ranges higher than $20 \mathrm{kHz}$ and keeping the difference between them lower than $20 \mathrm{kHz}$, the developed subtraction side band, $\mathrm{f}_{2}-\mathrm{f}_{1}$, will be in the audible range. For the development of talking structures, the difference between these frequencies has to be kept lower than $20 \mathrm{kHz}$. Instead of a continuous harmonic tone, modulated sound and even personalized messages, such as "loose bolt" or "damage at plate 1" can be generated and used for detection of defects.

\subsection{Experimental Setup}

Two experimental setups were used for implementation of the proposed SSHM approach in detection of loose bolts. The first SSHM system was prepared by holding two plates with four nuts and bolts. Also, a washer was designed to demonstrate the potential of the SSHM approach for general industrial implementations.

\subsubsection{Test with two plates held together with four bolts:}

Two aluminum plates with the thickness of $2 \mathrm{~mm}$ were held together by using four nuts and bolts (Figure 4.1). The dimensions of the top plate were $190 \mathrm{~mm}$ (length) by $150 \mathrm{~mm}$ (width). The bottom plate had the length of $400 \mathrm{~mm}$ and width of $190 \mathrm{~mm}$. The bolts were placed $25 \mathrm{~mm}$ away from the corners of the top plate. Two PZT disks were attached to the surface of the bottom plate for excitation of the system with two distinctive harmonic waves. The PZT elements were placed $84.5 \mathrm{~mm}$ away from the sides of the bottom plate. The diameter and the thickness of the PZT disks were $21 \mathrm{~mm}$ and $1 \mathrm{~mm}$ respectively. A USB data translation device (DT9832A) and an arbitrary signal generator (Rigol DG1022) were used for sending excitation signals to PZT1 and PZT2, respectively. 


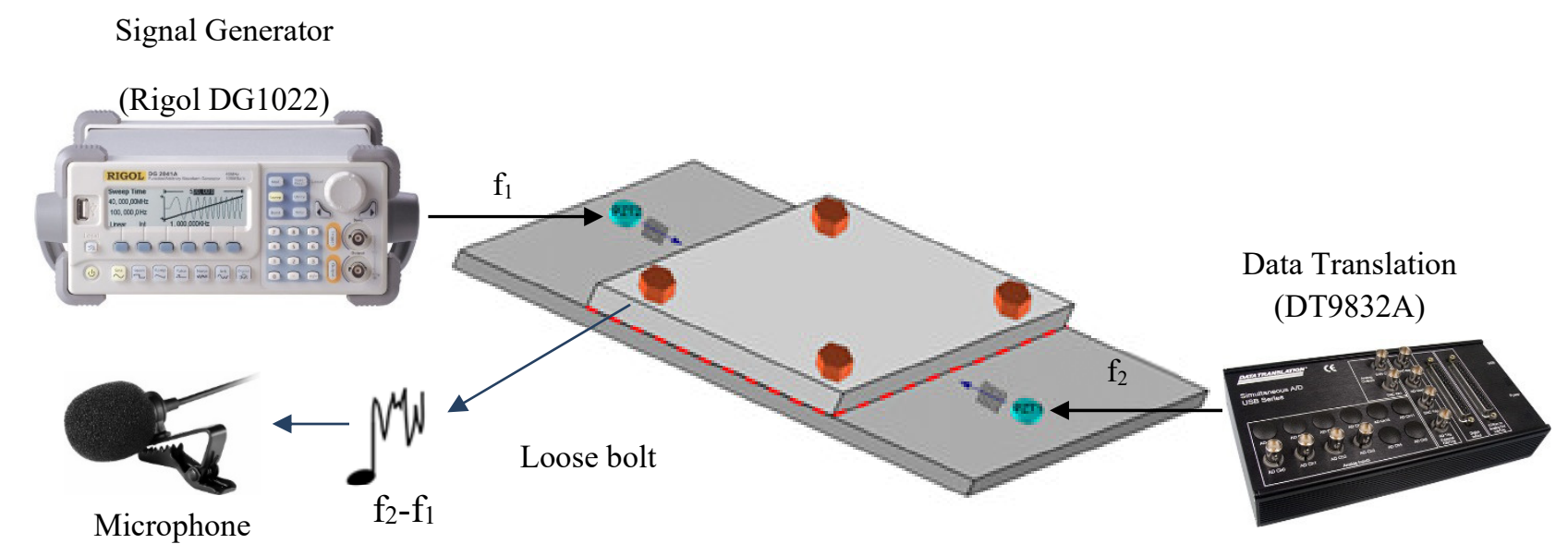

Figure 4.1 SSHM for detection of the loose bolt in the plates structure.

\subsubsection{Implementation of the SSHM in industrial washers}

Four washers were held together with a $25.8 \mathrm{~mm}$ long bolt with the diameter of $6.35 \mathrm{~mm}$ and a nut at the bottom (Figure 4.2). The two washers in the middle had the diameter of $50 \mathrm{~mm}$. For better distribution of tightening forces, the smaller washers with the diameter of $25 \mathrm{~mm}$ were located on the top and bottom of the larger washers. The thickness and internal diameters of all washers were $1 \mathrm{~mm}$ and $7.4 \mathrm{~mm}$, respectively. Two PZT actuator disks were attached to the surface of the top washer. The PZT disks had the diameter of 7 $\mathrm{mm}$ and the thickness of $0.5 \mathrm{~mm}$. It is possible to hold any number of plates together between the top two and bottom two washers. The proposed design is ready for any implementation from car lugs to bridge bolts. 


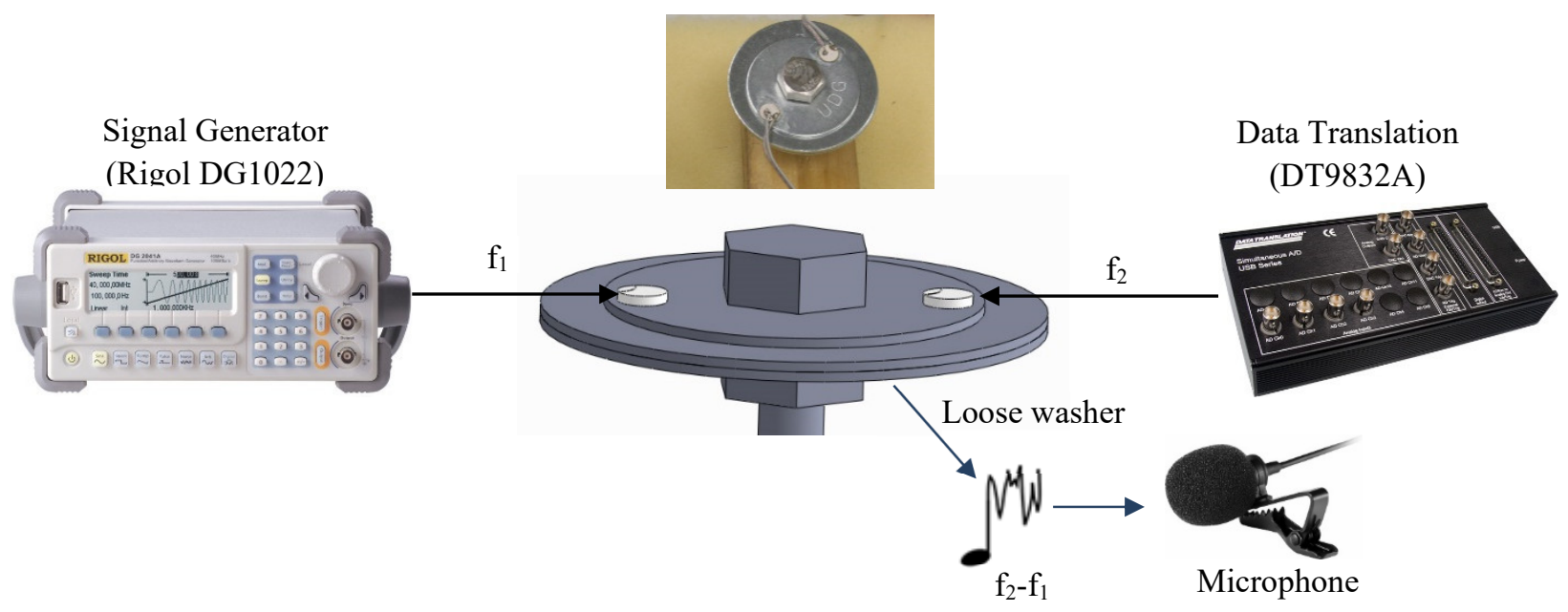

Figure 4.2 Schematic of the SSHM system for the washer system.

\subsection{Results and Discussion}

\subsubsection{Feasibility of the concept}

The goal of the proposed SSHM is to warn the operator with an audible alarm when the bolt of the washer or one of the bolts of the two plates is loose. In both experiments, two PZT disks were used as actuators. PZT1 was excited with a harmonic sine wave with the frequency of $80 \mathrm{kHz}$. PZT2 was excited with a single side modulated $10 \mathrm{kHz}$ beep sound with the same carrier frequency $(80 \mathrm{kHz})$. Thus, the difference between two signals, $\left(\mathrm{f}_{2}-\right.$ $\mathrm{f}_{1}$ ), is a $10 \mathrm{kHz}$ beep sound. For having a strong $\mathrm{f}_{2}-\mathrm{f}_{1}$ response, all excitation signals were amplified through Tektronix TM 504 amplifier up to 60 volts. The experiments were recorded by using a Canon $6 \mathrm{D}$ camera placed at $1 \mathrm{~m}$ distance from the setups. The tests were performed in the typical lab environment. The operation of these systems can be watched at the YouTube site of the FIU Mechatronics Lab at https://www.youtube.com/channel/UCCjMQVmrvWbnRO5Acb0OZlg. 


\subsubsection{Test with two plates held together with four bolts:}

The output of the microphone was recorded for 16 seconds and is shown in Figure 4.3. Initially, one of the bolts was loose and the nonlinear system created the audible alarm at the difference frequency, $10 \mathrm{kHz}$ beep sound. Then, the operator started to tighten the loose bolt at the 6 th second and finished the task at the 8 th second. Due to the tight contact and diminishing of the nonlinear behavior, the sound immediately diminished after the 8th second. The experiments demonstrated the feasibility of the low-cost SSHM systems.

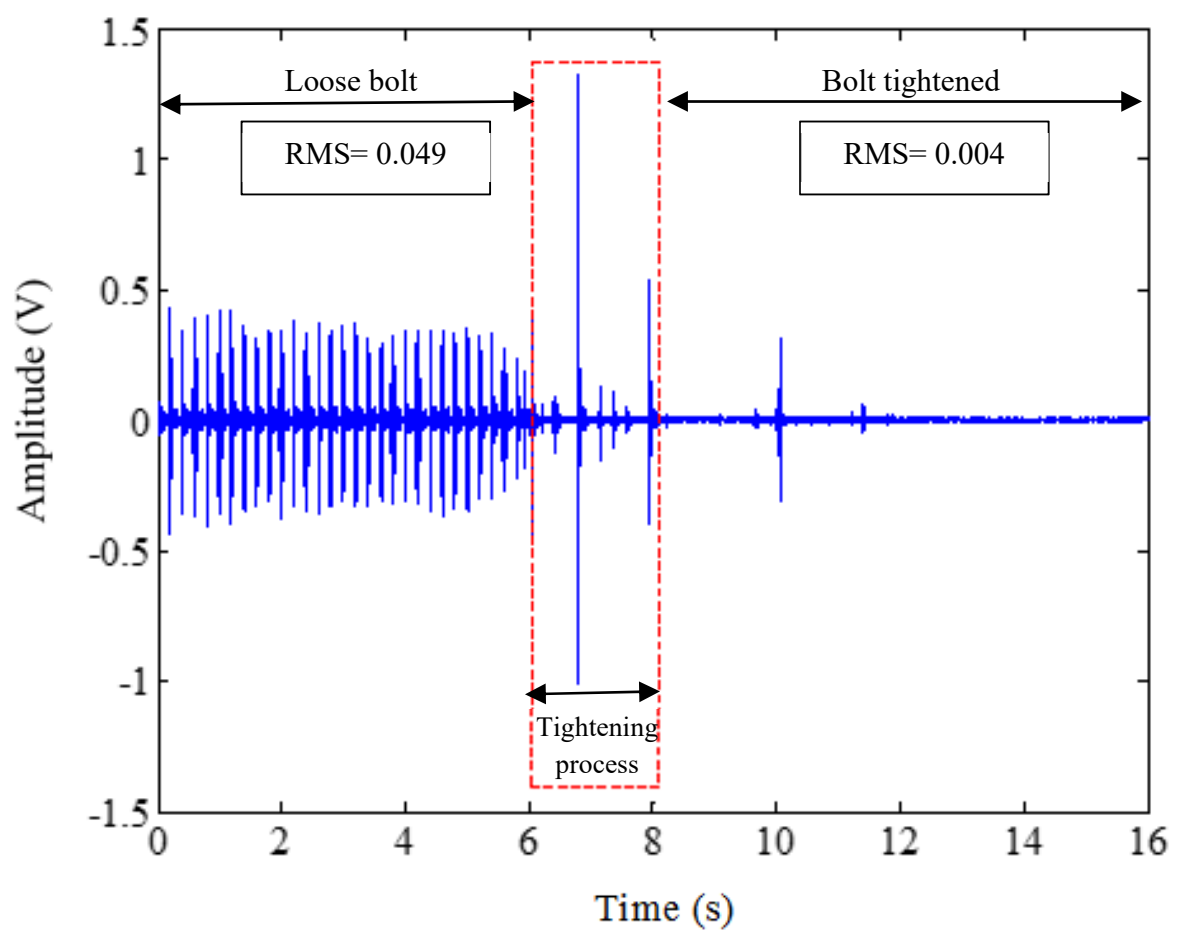

Figure 4.3 Recorded response of the plate system to the excitation.

\subsubsection{Implementation of the SSHM with industrial washers:}

The variation of the microphone output for the washer system is presented in Figure 4.4, for a time span of 30 seconds. In the experiment, initially, the bolt was loose. Therefore, 
the $10 \mathrm{kHz}$ beep sound $\left(\mathrm{f}_{2}-\mathrm{f}_{1}\right)$ was generated due to the nonlinearity formed by the light contact between washers. The loose bolt was then tightened in between 5th and 7th seconds. Then, right after tightening the audible beep sound diminished due to the tight contact. The bolt was again loosened in between 23rd and 25th seconds and as expected the loud beep sound was started again.

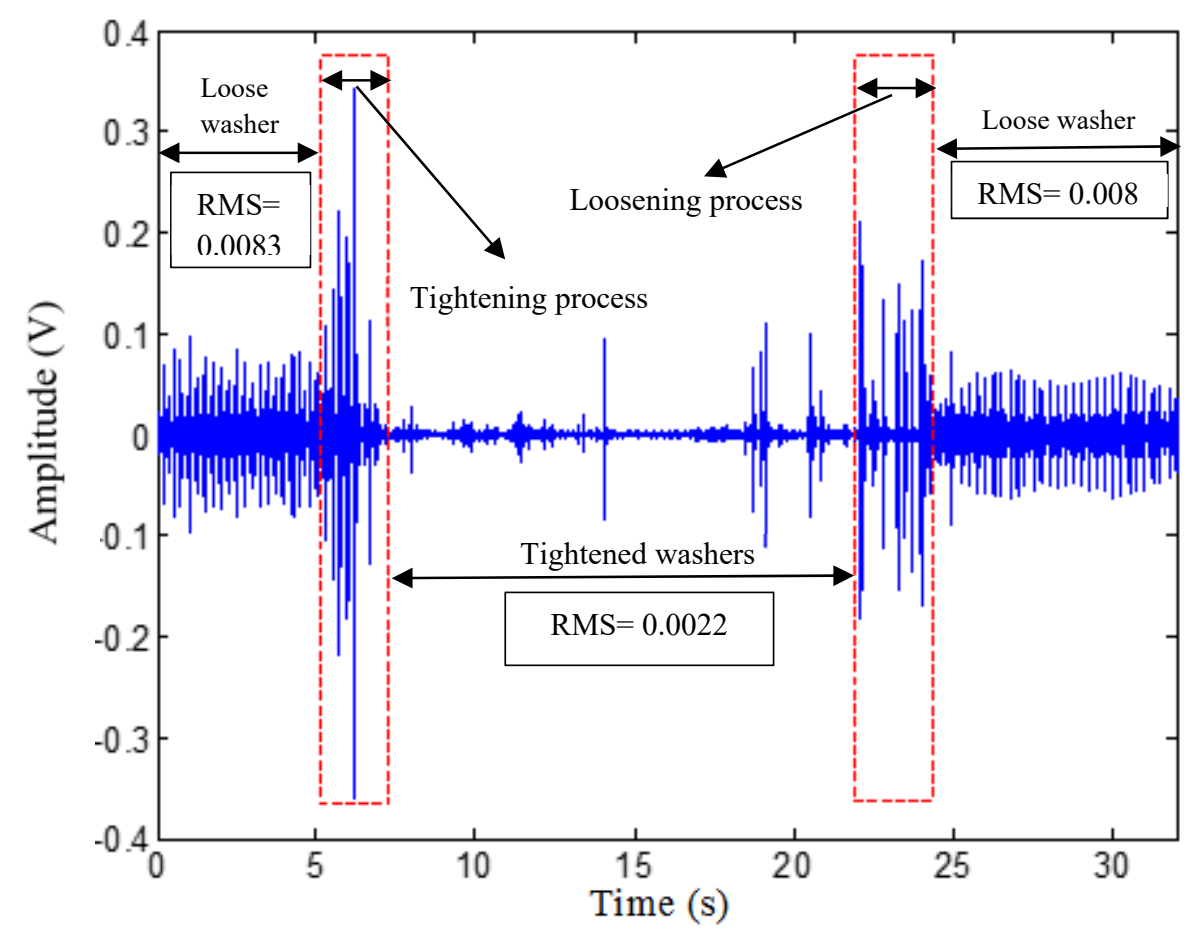

Figure 4.4 Recorded response of the washer system to the excitation.

As can be seen from the figures, the light contact between washers and plates works as a nonlinear mixer for excitation waves and produces the audible $f_{2}-f_{1}$ element. In the next section, different torques were applied for tightening of the bolt of the washer system to examine the resultant change in the amplitude of the subtraction element. 


\subsubsection{Quantification of bolt tightness using the SSHM method}

The correlation between the applied torque on the bolts and the sound level which corresponds to the amplitude of the audible subtraction element was studied in this section. In the previous experiments, the signals were amplified up to 60 volts. However, in this section, the input signals to the piezoelectric elements were reduced to $20 \mathrm{Vpp}$ to eliminate the need for the amplifiers. To obtain a loud beep sound while using the lower excitation voltage, first finding an effective combination of the carrier and modulation frequencies was investigated.

The first experiment was carried out to determine the carrier frequency with the highest amplitude. For that purpose, PZT1 was excited with a $20 \mathrm{Vpp}$ sweep sine wave in the range of 50-250 kHz and the response was collected from the PZT2 element with the sampling frequency of $1 \mathrm{MHz}$. The FFT of the response of the washer system is shown in Figure 4.5. The highest peak was observed at the frequency of $117800 \mathrm{~Hz}$. Therefore, this frequency was chosen as the carrier frequency.

The second step was to choose the effective frequency for the beep sound. An audible sweep sound in the range of $100 \mathrm{~Hz}-20 \mathrm{kHz}$ and on/off duration of $0.5 \mathrm{sec}$ was single side modulated with the $117,800 \mathrm{~Hz}$ carrier frequency. The plot of audible sweep excitation frequency is shown in Figure 4.6 and the FFT of the single side modulated signal is shown in Figure 4.7. PZT1 was excited by a pure $117,800 \mathrm{~Hz}$ signal and the modulated signal was applied to PZT2. Therefore, the difference between excitation frequencies was a sweep sine in the range of $100 \mathrm{~Hz}-20 \mathrm{kHz}$. 


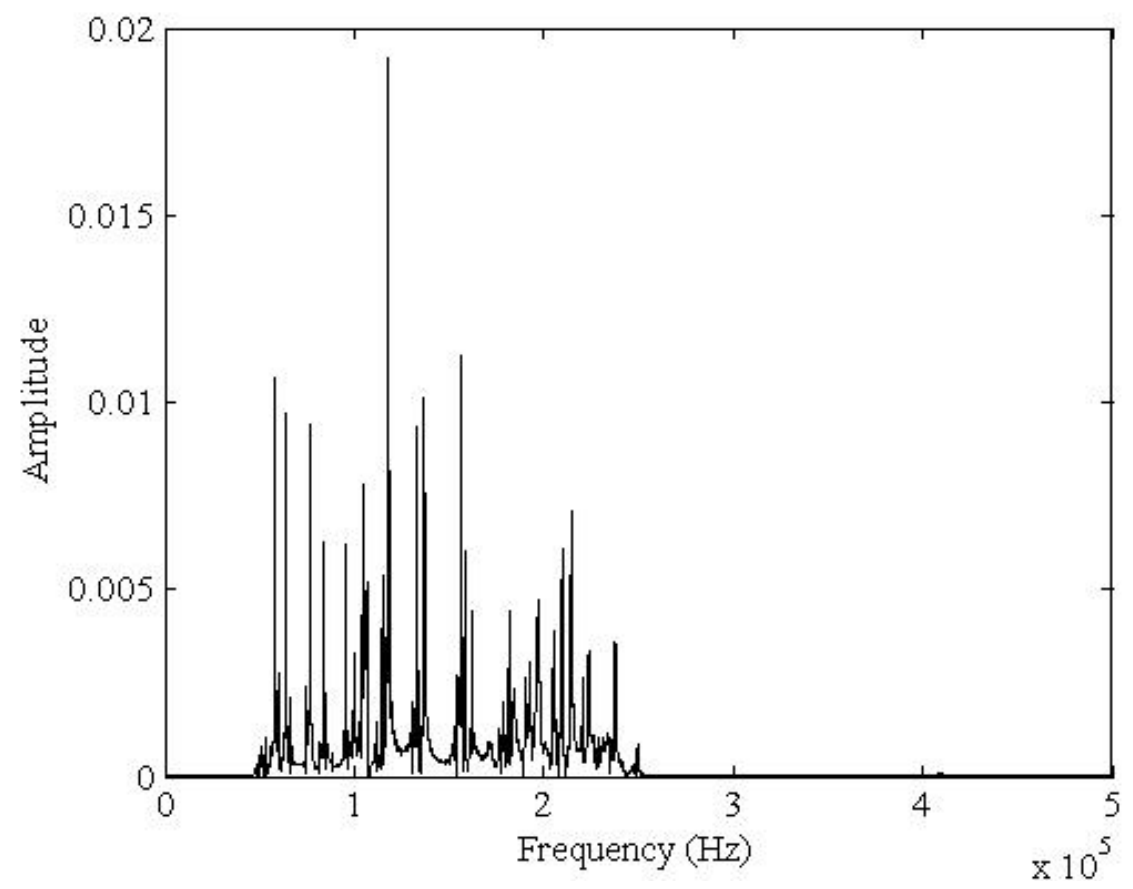

Figure 4.5 The FFT of the response of the washer system to the sweep excitation in the range of $50-250 \mathrm{kHz}$.

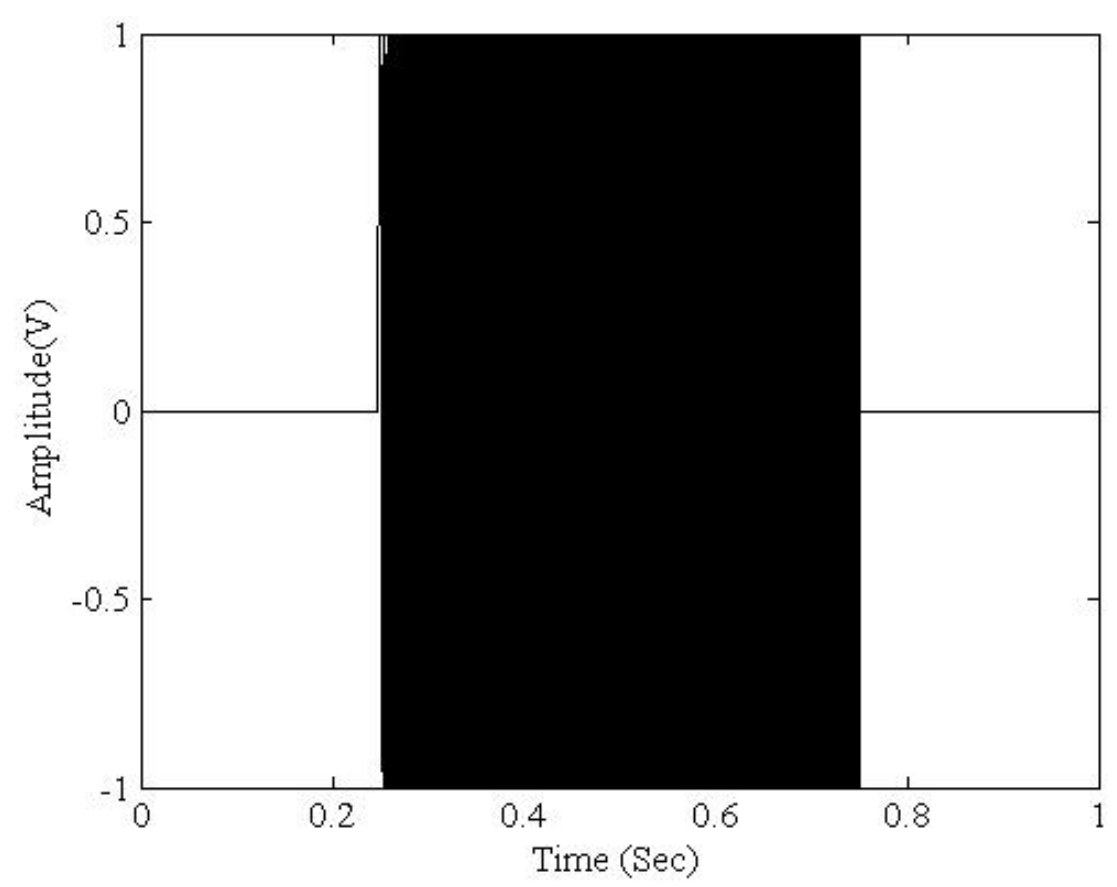

Figure 4.6 The audible sweep excitation in the range of $100 \mathrm{~Hz}-20 \mathrm{kHz}$. 


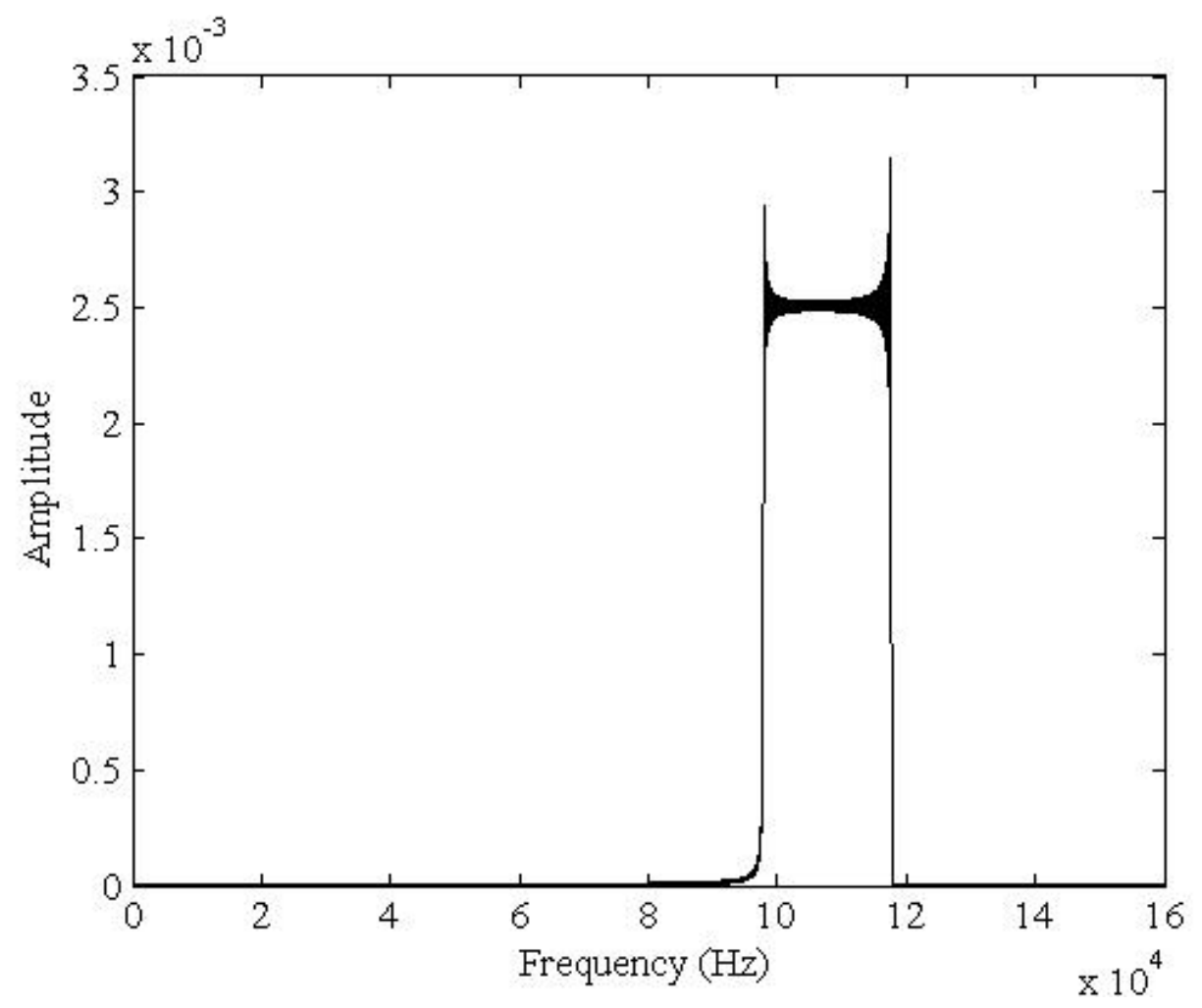

Figure 4.7 The FFT of the applied modulated signal.

The response of the washer system to the bitonal excitation was recorded with the microphone placed at 1 inch distance from the specimen with the sampling frequency of 44.1 kHz. The response and its FFT are shown in Figure 4.8 and Figure 4.9, respectively. Figure 4.9 shows that the response of the structure to the bitonal excitation has higher amplitudes in some frequencies. In this study, we chose $12,320 \mathrm{~Hz}$ frequency for further study. This frequency has the maximum amplitude in the FFT response in the range of 10$15 \mathrm{kHz}$ which can be easily heard by most people. 


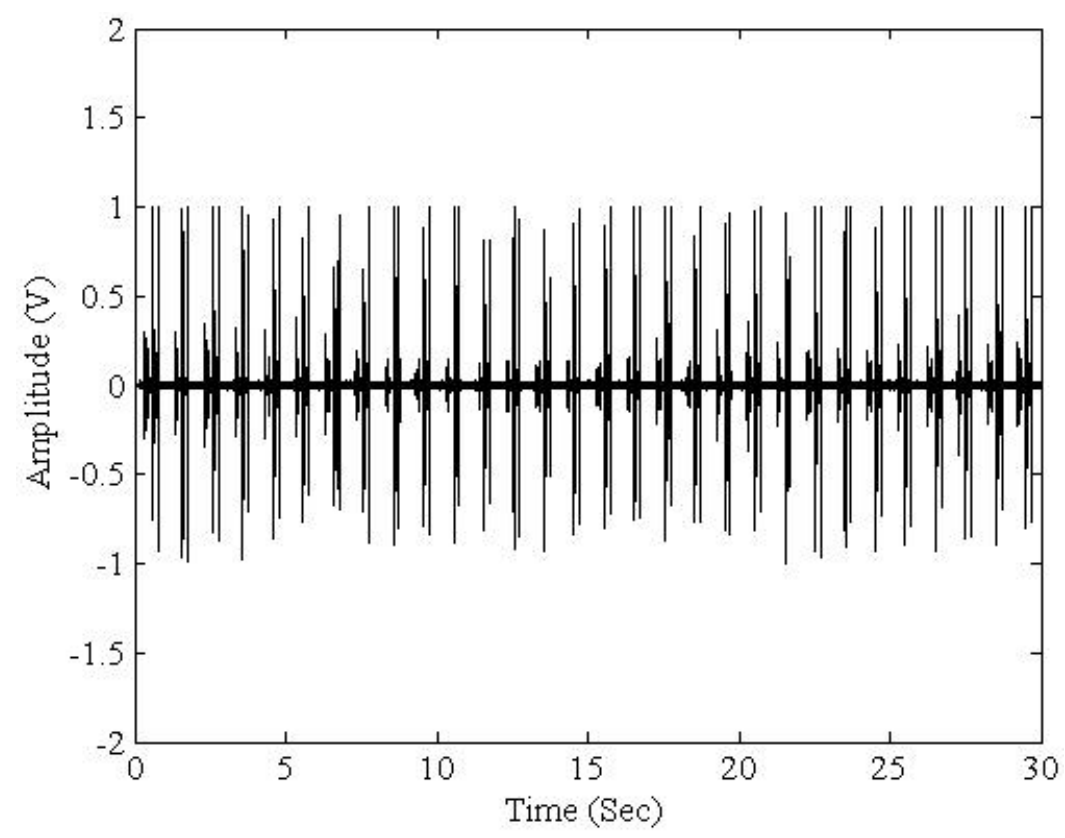

Figure 4.8 The recorded audible response.

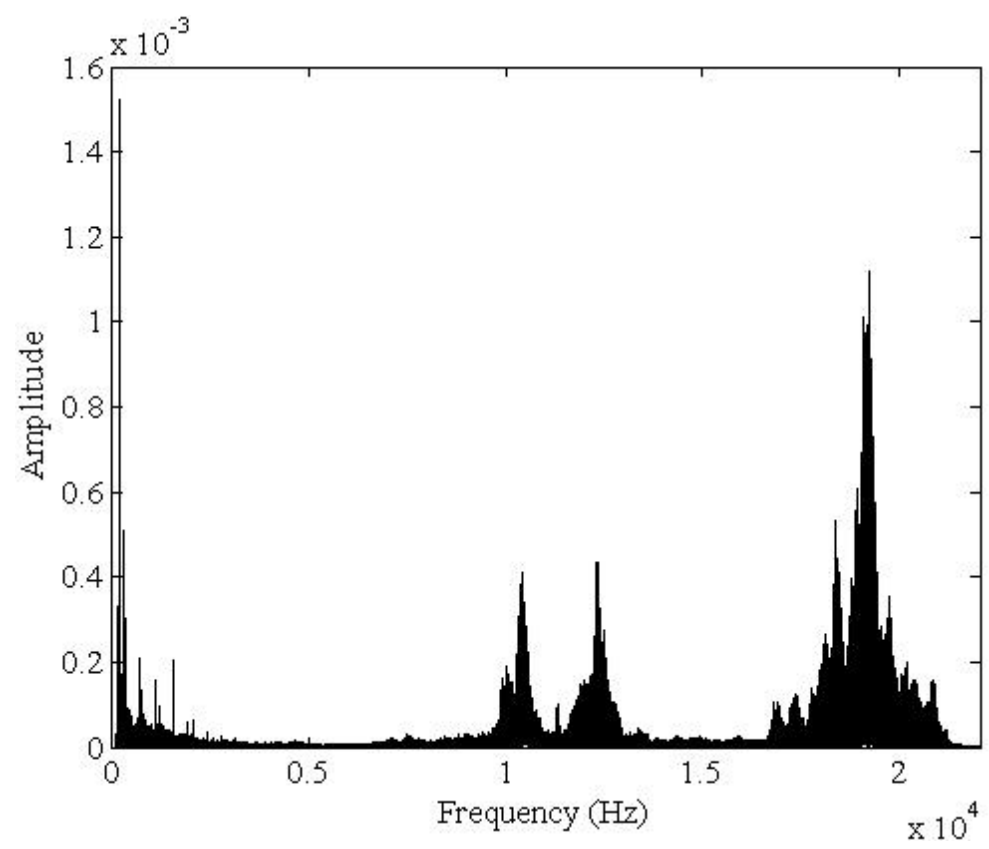

Figure 4.9 The FFT of the recorded response.

In the third step, a harmonic signal with the $12,320 \mathrm{~Hz}$ frequency was modulated with the $117,800 \mathrm{~Hz}$ frequency and applied to PZT2. PZT1 was excited with a pure $117,800 \mathrm{~Hz}$ sine 
wave. So, the difference between the two excitation frequencies was the $12,320 \mathrm{~Hz}$ beep sound. Using the same bitonal excitation, the sound was recorded when the washer was tightened at different torque levels. This recording is presented in Figure 4.10. Also, the FFT values of the responses are shown in Figure 4.11. Figure 4.10 and Figure 4.11 demonstrate that increasing the applied torque reduced the amplitude of the audible sound. It should be noted that there is a significant difference between the response of the loose washer and tightened washers while the difference between the response of the washers with torques higher than $2 \mathrm{Nm}$ is negligible and insignificant. For a better illustration of the correlation between the tightening torque and amplitude of the sound level, experiments were repeated for three times and the average RMS values of the three experiments at different torques are presented in Figure 4.12.

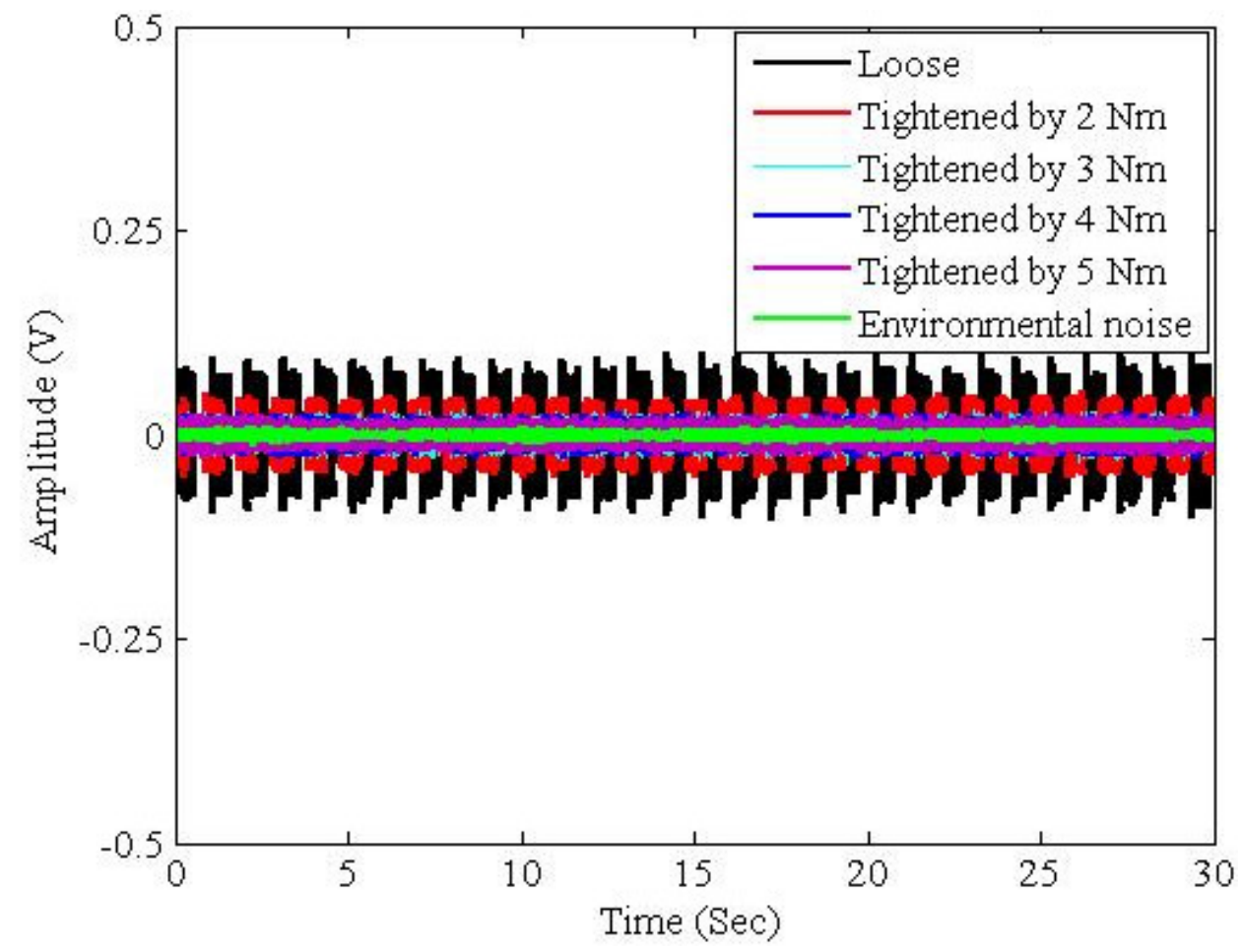

Figure 4.10 The comparison between audible response of the washer system in different torques. 


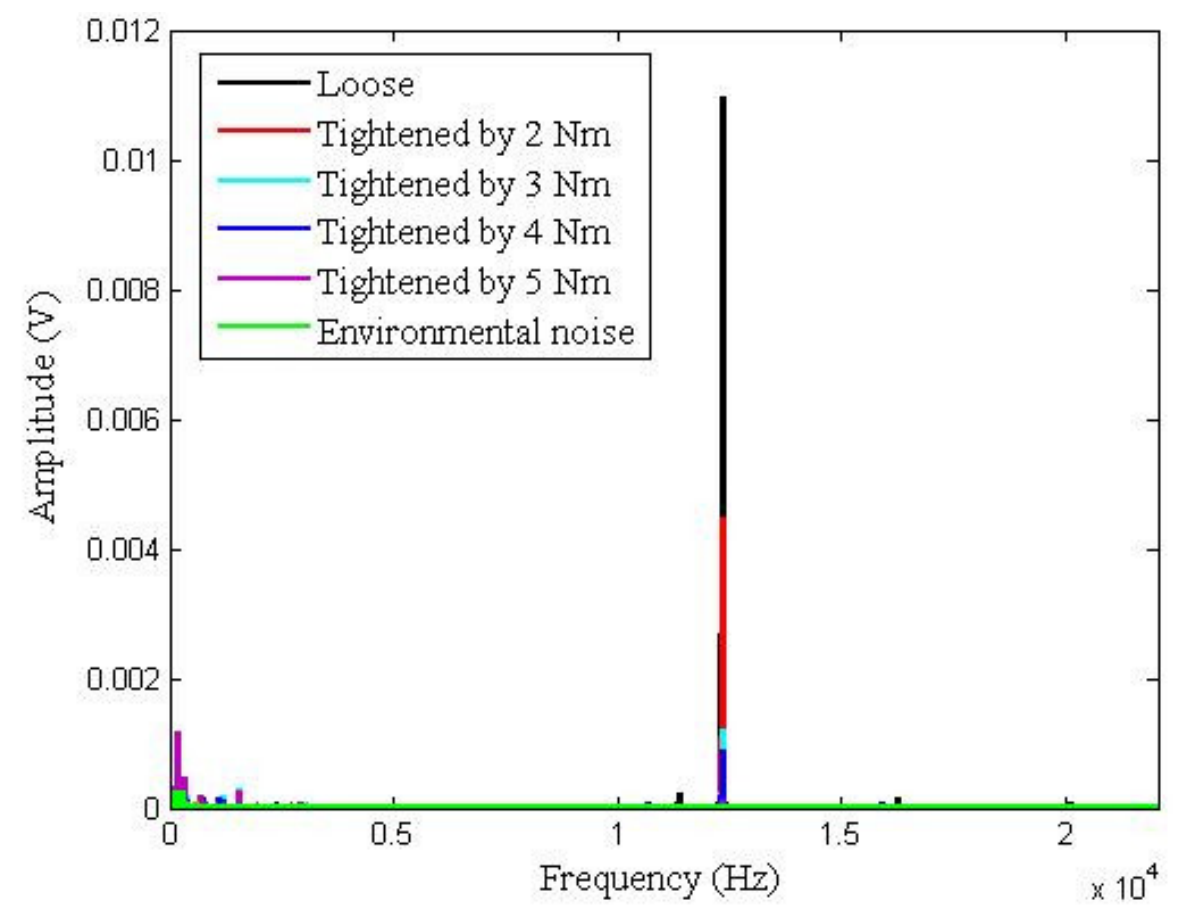

Figure 4.11 The comparison between the FTT values of the audible responses of the washer system in different torques.

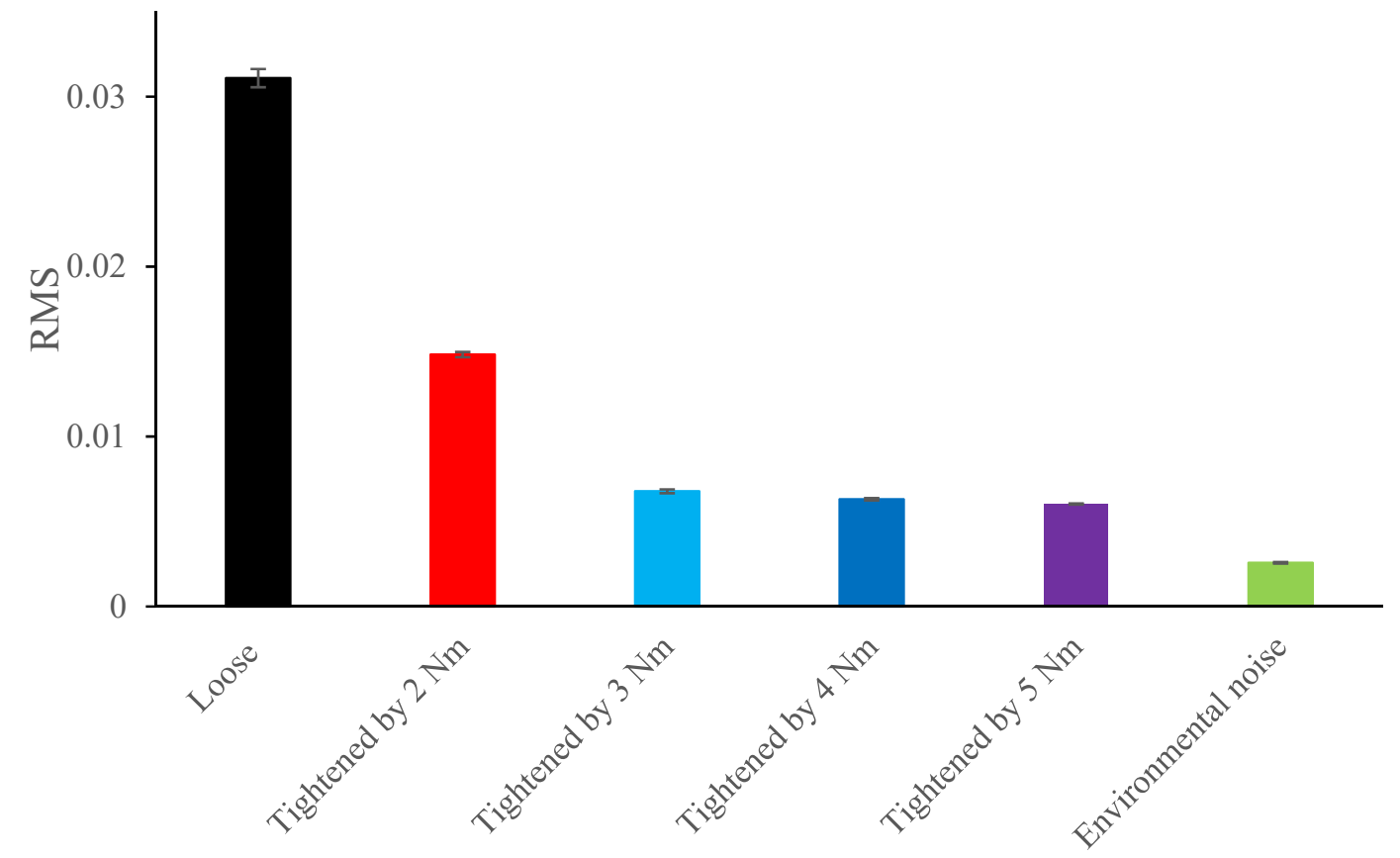

Figure 4.12 The comparison between the RMS values of the audible responses of the washer system at different torques. 
The same washer system was then used with plates. The same excitation signals with the same characteristics were applied at the previously tested torques. The time domain response, FFT, and RMS values are shown in Fig. 13- Fig.15, respectively. These figures show that the proposed SSHM system worked effectively when the washers hold the plates. The SSHM system produces a louder sound when the washer is tightened with less torque.

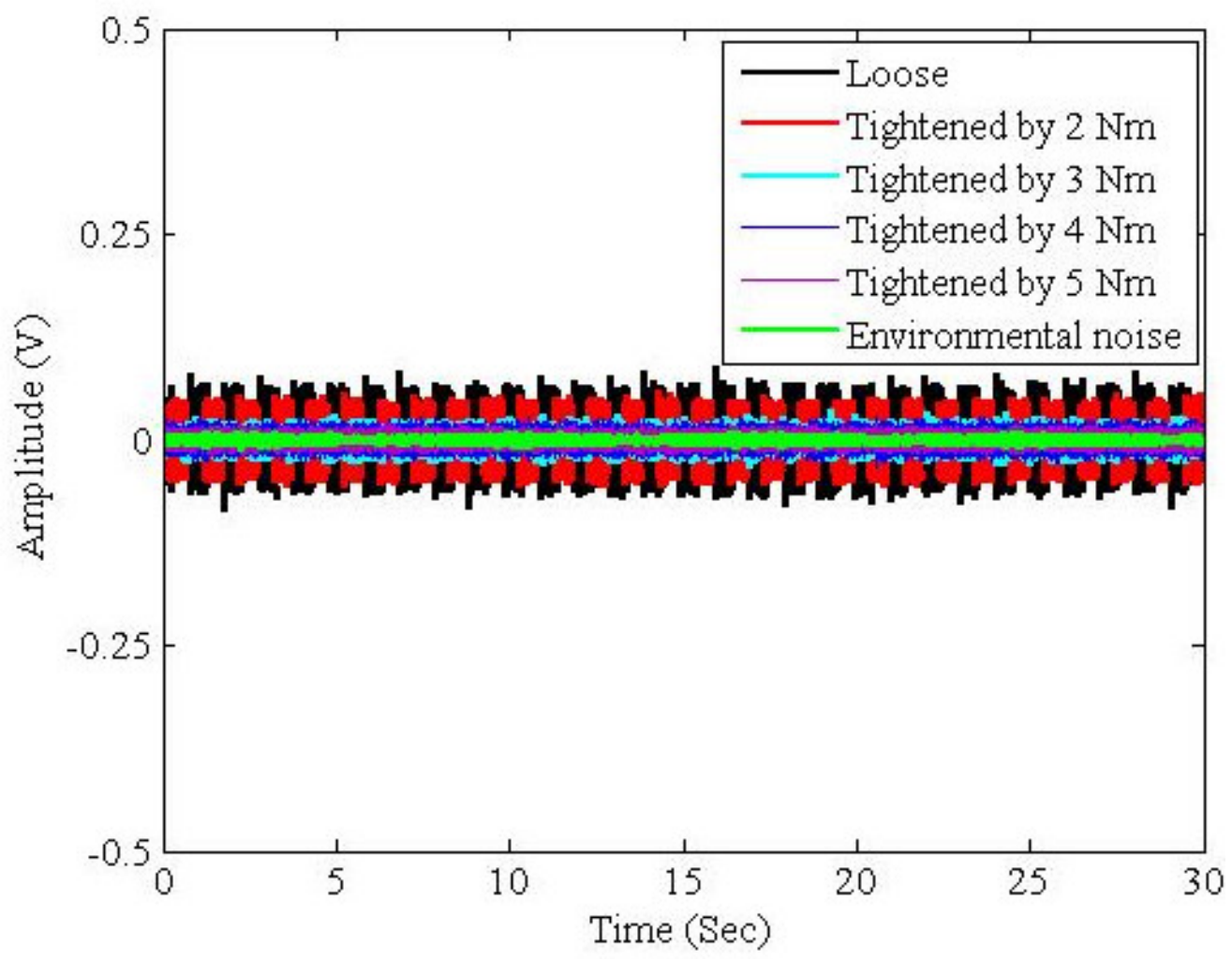

Figure 4.13 The comparison between the RMS values of the audible responses of the washer installed in the plate system in different torques. 


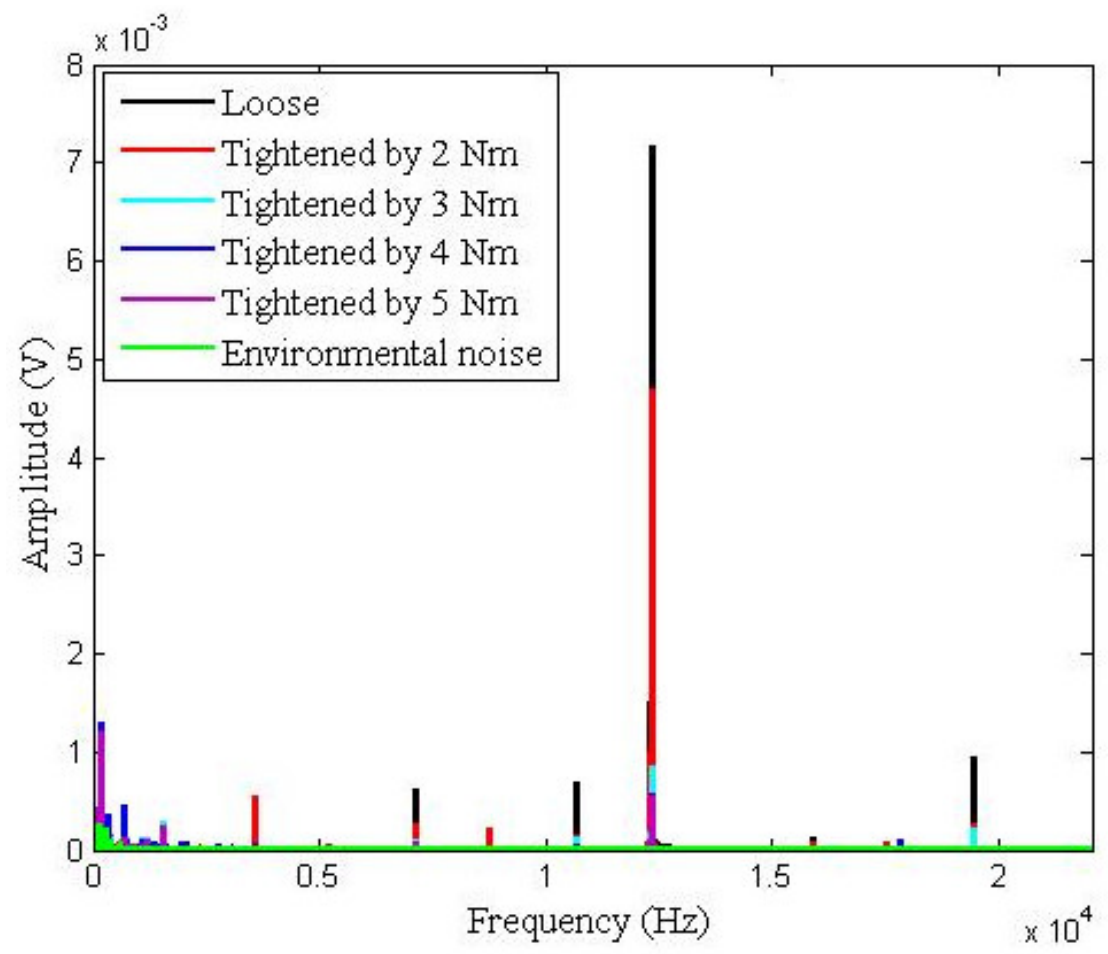

Figure 4.14 The comparison between the FTT of the audible responses of the washer installed in the plate system in different torques.

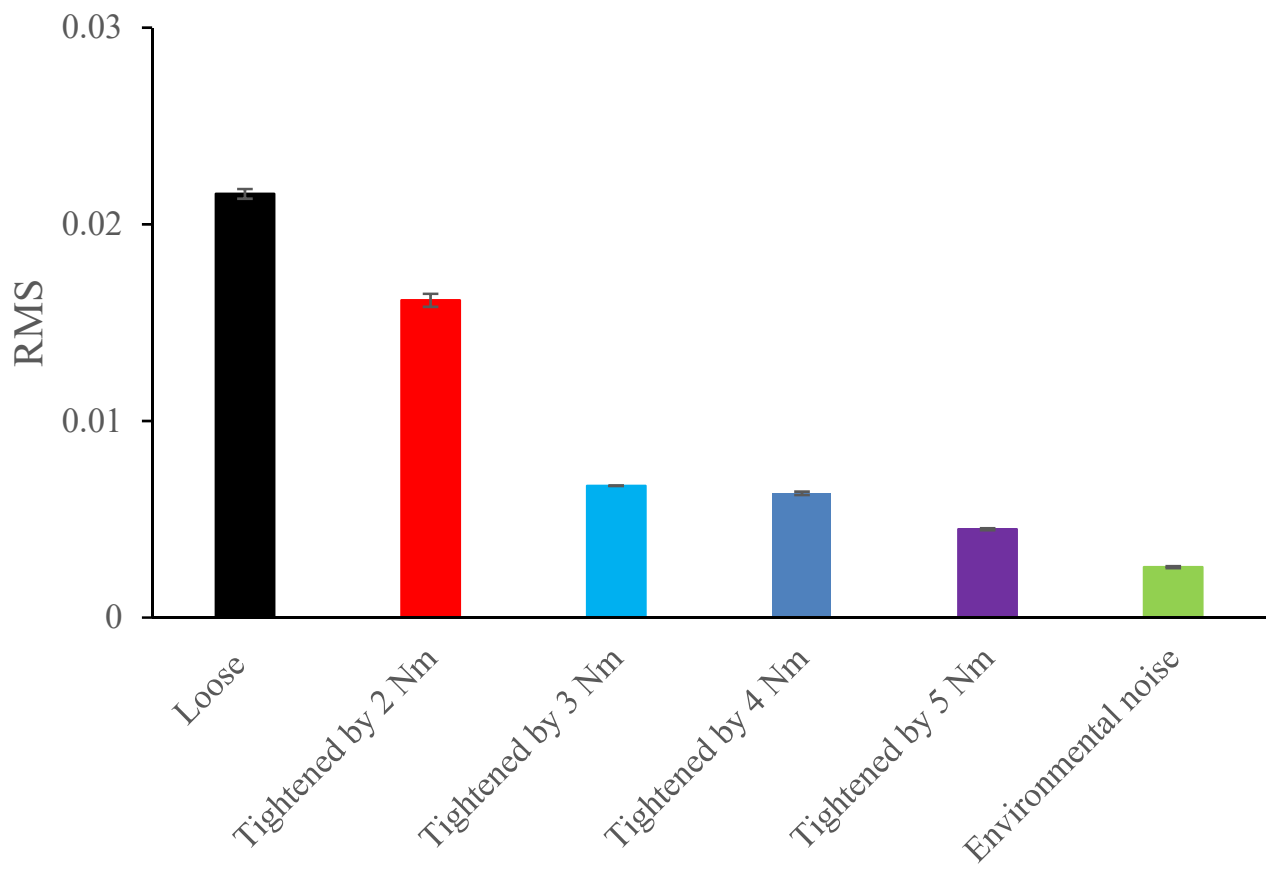

Figure 4.15 The comparison between the RMS values of the audible responses of the washer system installed in the plate system in different torques 


\subsection{Summary}

Nonlinear wave modulation spectroscopy, impact modulation, and other similar methods monitor the formation of newer frequencies in the response of structures to the bitonal excitations. These methods, other nonlinear approaches, and conventional SHM systems use sensors, data acquisition devices, processors, and skilled technicians. The cost and complexity of the implementation of SHM systems have limited their applications to monitoring of critical structures.

In this study, the very first SSHM system was introduced to propose a low cost alternative to the SHM systems. The SSHM system was used for detection of the loose bolt when two plates were held together with bolts. The loose bolt of a washer was also detected with the same approach. The system uses two distinct excitation signals while the frequency difference between excitation signals was kept in the audible range. Such bitonal excitation triggers an audible alarm at the location of the loose bolt. The frequency of the sound is equal to the difference of the excitation signals. In the experiments, the SSHM system created substantially louder audible alarms in the presence of the loose bolt, without any sensors, processors and user interface.

The proposed SSHM approach can be used independently or can be conveniently integrated into the conventional active SHM systems as a backup module. The proposed SSHM back up module may warn the operators at the site of the defective structure with sound when the SHM system collapse because of the failure of one of its components. Instead of the beep sounds created by modulation of harmonic signals, verbal statements may be modulated to give meaningful messages to the operator at the location of loose 
bolts. The proposed SSHM approach may be used for detection of different types of defects such as delamination, debonding, and cracks as long as they transform the linear characteristics of the structure into the nonlinear characteristics. 


\section{CHAPTER 5}

\section{Localization of Multiple Defects Using the Compact Phased Array (CPA)}

\section{Method}

Array systems of transducers have found numerous applications in detection and localization of defects in structural health monitoring (SHM) of plate-like structures. Different types of array configurations and analysis algorithms have been used to improve the process of localization of defects. For accurate and reliable monitoring of large structures by array systems, a high number of actuator and sensor elements are often required. In this study, a compact phased array system consisting of only three piezoelectric elements is used in conjunction with an updated total focusing method (TFM) for localization of single and multiple defect in an aluminum plate. The accuracy of the localization process was greatly improved by including wave propagation information in TFM. Results indicated that the CPA approach can locate single and multiple defects with high accuracy while decreasing the processing costs and the number of required transducers. This method can be utilized in critical applications such as aerospace structures where the use of a large number of transducers is not desirable.

\subsection{Introduction}

Guided-waves based structural health monitoring systems are widely used in civil, mechanical, and aerospace applications. Guided waves, can propagate over long distances and inside curved walls with low attenuation and high sensitivity [133]. In particular, Lamb wave based methods play a critical role in detection and localization of damage in SHM of pipes and plate-like structures. These waves are utilized in transducer arrays for localization of defects since they can scan large areas and provide a mapping of defects of 
the scanned surface. Typically, complicated electrical devices and/or detection algorithms are required for successful damage detection and localization using transducer arrays.

Generally, in SHM, detection and localization are carried out based on information that is collected from the time of flight, energy transmission, reflections from defects, changes in wave modes, and electro mechanical impedance information [134], [135]. Regarding transducer arrays, a single transducer pair is not enough to determine the exact location of damage in plate-like structures. Transducer arrays are typically used to obtain a map of defects in a structure. Different algorithms and approaches can be used for this purpose.

Among the array systems, the sparse transducer method is highly suitable for large but simple structures [136], [137]. In this method, transducers are mounted in different locations on the surface of the target structure. Typically, at least three or four sensors are required to find the location of the damage [138]-[141]. In the sparse approach, multiple numbers of tests have to be performed, where in each test, one of the piezoelectric elements acts as an actuator and the remaining piezoelectric elements work as sensors. A virtual image of possible locations of damage on the target surface can be formed from all sensors. The image contrast can be negatively influenced by low signal to noise ratio (SNR), direct arrivals, scattered waves, and reflections from boundaries. Regarding the low SNRs, it should be noted that the excitation is omnidirectional and emitted waves propagate in all directions. As a result, a very small portion of energy is radiated in each direction and therefore, reflections from a damage location to the sensors will be very low in energy. Thus, in most of its applications a high voltage was applied for the excitation to increase the SNR value. For instance, Konstantinidis and Prado used peak-to-peak voltages of 190 
$\mathrm{V}$ and $120 \mathrm{~V}$ for the excitation [142], [143]. However, baseline subtraction can improve the resolution and alleviate the problem [144]. Yet, the sparse arrays are not suitable for small and complex structures which do not have enough space for placement of transducers.

When dealing with smaller structures, the compact array configuration can be an alternative for sparse arrays. Various arrangements of compact transducer arrays, such as circular, rectangular, and linear configurations have been proposed to minimize the footprint of transducers on small structures. These arrays generally use a single transmitter and multiple receivers (STMR) [145]. Different algorithms, such as the phase reconstruction algorithm [146], minimum variance distortion-less response (MVDR) [140], [147], and total focusing method (TFM) [148], [149] have been used in the compact array systems. These arrays can also work as multi-transmitter multi receiver (MTMR) systems [150].

Phased arrays are another group of methods in array based SHM systems which work like radar systems. In these methods, real beam forming is carried out by steering the outputs of all transducers in a controlled manner to trace the whole surface of a structure similar to a radar system. That is, in this approach through real beam forming a powerful wave energy is emitted in a predetermined direction by stimulating all the actuators at the same time. Short inspection times and high SNR values are the main advantages of this method. In linear ultrasonic phased arrays focusing the energy in the desired directions requires expensive and complex electronic phase or pulse shifters and multiplexers for excitation of all transducers at the same time [151]-[153].

Virtual beam forming methods were developed to resolve difficulties regarding real beam forming and simultaneous excitation of transducers. In virtual beam forming the 
transducers are excited one at a time and there is no need for the concurrent excitation of all transducer elements. The recorded responses in different sensor locations are post processed to obtain the required information in a desired direction by filtering the unwanted information related to the other directions [143], [154]. However, massive post processing and low SNR value are the two main disadvantages in the group of virtual beamforming or steering based techniques.

This study proposes the application of an incremental three stepped steering approach using a compact phased array (CPA) with total focusing method. The proposed method eliminates the need for multiplexers, pulse, and phase shifter devices that are used in phased array systems. The method is based on creating beams in desired directions by using two transducers at a time and a third PZT element for sensing. The results are then processed by using the standard and modified TFM. Finally, by adding the information collected from all directions, a complete map of defects can be acquired. The theoretical background, experimental setup, and the performance of the proposed SHM system will be discussed in the following sections.

\subsection{Theoretical Background}

\subsubsection{Lamb waves}

Elastic mechanical waves can be efficiently produced on plate-like structures using piezoelectric elements. These waves are referred to as guided waves since they are guided and bounded by two surfaces of the plate. Vertically polarized ultrasonic guided waves that travel in plates are called Lamb waves. In plate structures, Lamb waves can propagate in asymmetric and anti-symmetric modes. Symmetric and antisymmetric Lamb waves 
have symmetrical and anti-symmetrical movements about the mid-plane of the plate, respectively. The wave propagation speed is not necessarily the same for symmetric and anti-symmetric modes even at the same frequencies. The velocities of the waves can be calculated when the excitation frequency and the plate thickness are known. The characteristics of the Lamb waves depend on the excitation frequency, access angle, dimensions of the plate, transducer's characteristics and the material of the structure [29].

\subsubsection{Array radiation pattern}

Transmission of the waves produced by phased arrays was first studied in 1905 for enhancing transmission of radio waves during World War II. Since that time, phased arrays have been used in many areas, such as ultrasonic applications, optics, and acoustics. In general, array configurations consist of multiple transducer elements that are carefully arranged to achieve a desired radiation pattern. The radiation pattern of a linear array made by identical transducer elements can be found according to the pattern multiplication theorem of antenna arrays [155].

$$
\text { Array's pattern }=\text { element's pattern } x \text { Array factor }
$$

The element's pattern is the pattern of a single element in the array. By considering that circular PZTs act as omnidirectional sources, the element pattern has a fixed value, one which doesn't depend on the diameter of the PZTs [156]. That is, the array factor is a function of the geometry of the array and the excitation characteristics, such as amplitude and phase of the elements. For a linear array consisting of $\mathrm{N}$ isotropic radiator elements, the radiation pattern can be described as: 


$$
\begin{gathered}
A(\psi)=\frac{\sin \left(\frac{N \psi}{2}\right)}{N \sin \left(\frac{\psi}{2}\right)} e^{j(N-1) \psi / 2} \\
\psi=k d \cos (\phi)
\end{gathered}
$$

where $\phi$ is the angle between the propagation line and array line, $d$ is the distance between elements of the array, and the $k=2 \pi / \lambda$ is the wave number. The normalized power gain of an array is then:

$$
g(\phi)=|A(\psi)|^{2}=\left|\frac{\sin (N \psi / 2)}{N \sin (\psi / 2)}\right|^{2}=\left|\frac{\sin ((N k d / 2) \cos \phi)}{N \sin ((k d / 2) \cos \phi)}\right|^{2}
$$

Figure 5.1 shows the effect of different distances between elements on the resultant radiation pattern of a two element array system.

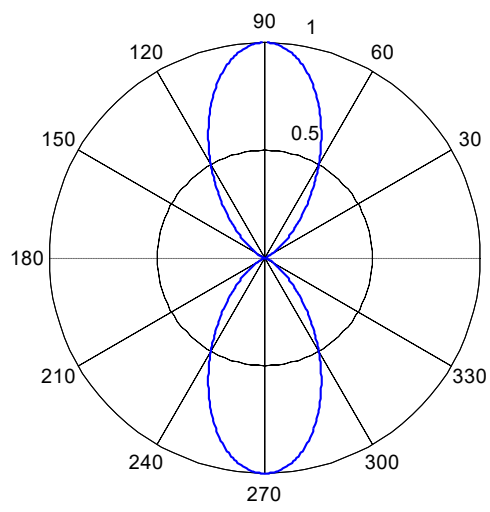

a)

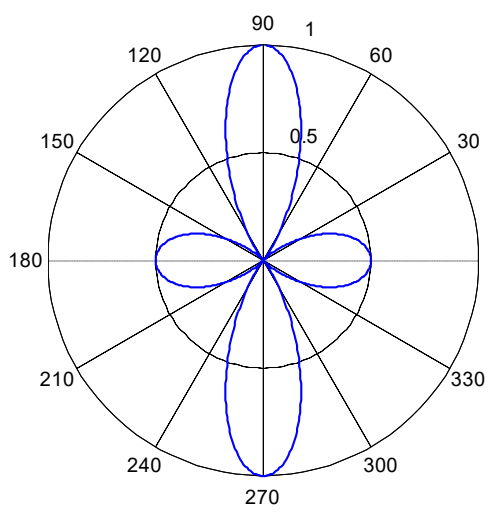

b)

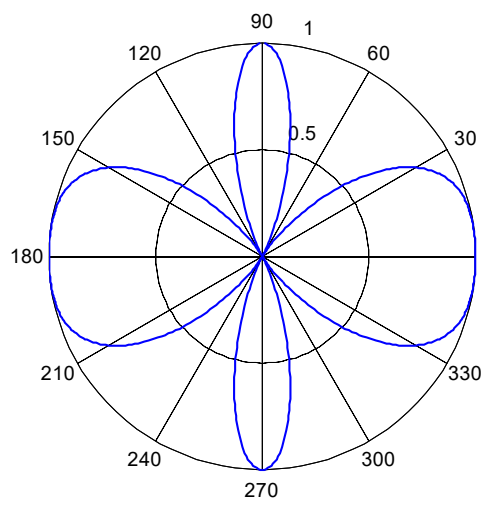

c)

Figure 5.1 Resultant radiation pattern of a two-element linear phased array system for different element spacing: a) $\mathrm{d}=0.5 \lambda$ b) $\mathrm{d}=0.75 \lambda c) \mathrm{d}=\lambda$. 
As can be seen from Figure 5.1, when using the half wave length, all of the energy is efficiently imparted along the two main lobes without formation of any other side lobes. For actual rotation of the main lobes in a desired direction proper time/phase delays must be applied in between excitations. This occurs by complex phase or pulse shifters and multiplexers. To keep the number of actuators as small as possible and to avoid steering of the main lobes for complete coverage, this study uses a three element compact phased array system that provides a complete coverage in three steps.

The radiation pattern shown in Figure 5.1 is for single tone excitation sources. But many SHM systems use windowed sine waves which have slightly different frequency band spectrum characteristics. Figure 5.2 shows a five cycle hamming windowed sine wave and its normalized frequency spectrum. The phase speed of the Lamb wave can also vary with the operating frequency and the material properties. Phase speed of the A0 mode for an aluminum plate (Al-2024) is shown with the dashed line in Figure 5.2 (b). In that case, wave number $k$ in equation (5.2) is a function of the wave phase speed and the frequency. The total solution is the summation of the radiation pattern of all frequency components (equation (5.3)).

$$
g_{T}(\phi)=\sum_{j=1}^{N} E(j) g(\phi, j)
$$

Where $E(j)$ is the amplitude of the Fourier transform of the excitation signal and $N$ is the number of frequency components. 
In Figure 5.3, the red line shows the radiation pattern of a two-element linear phased array excited with five cycles of a hamming windowed sine wave with a frequency of $100 \mathrm{kHz}$. The blue line shows the pattern of the continuous $100 \mathrm{kHz}$ excitation. As can be seen from the figure, there is a very small difference between the two patterns. Thus, the single tone approach can be safely used for simplification of the calculation while the dispersion effect is very negligible. For far-off damage in large structures, dispersion correction might be applied to further improve the sensitivity of the method and compromising for the effect of dispersion in the calculations [157].

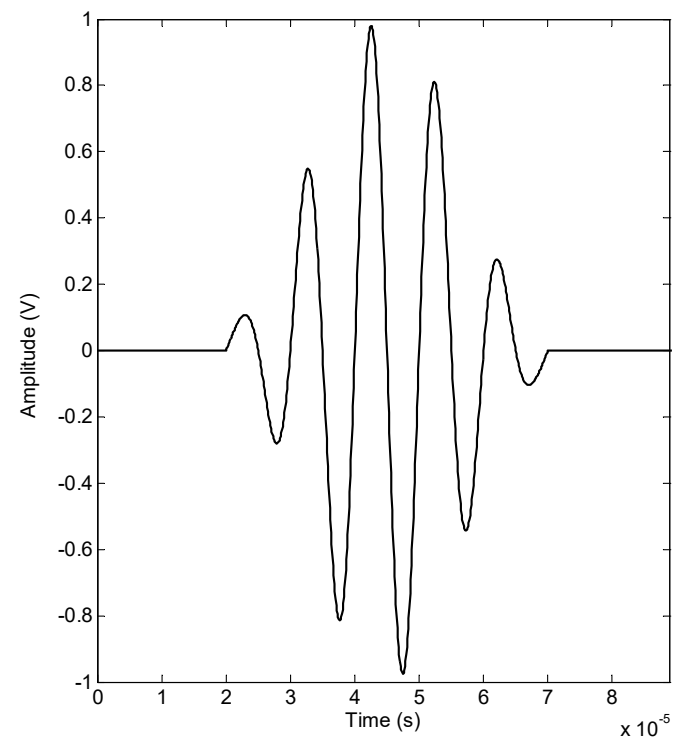

a)

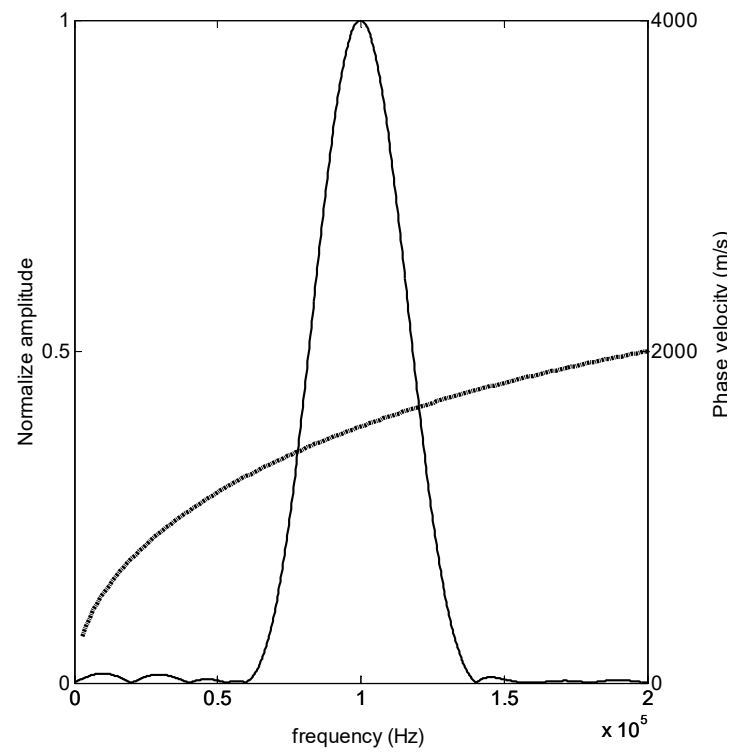

b)

Figure 5.2 a) Excitation signal (five cycle hamming windowed sine wave) b) frequency spectrum and phase speed at A0 mode for the test plate (Al-2024). 


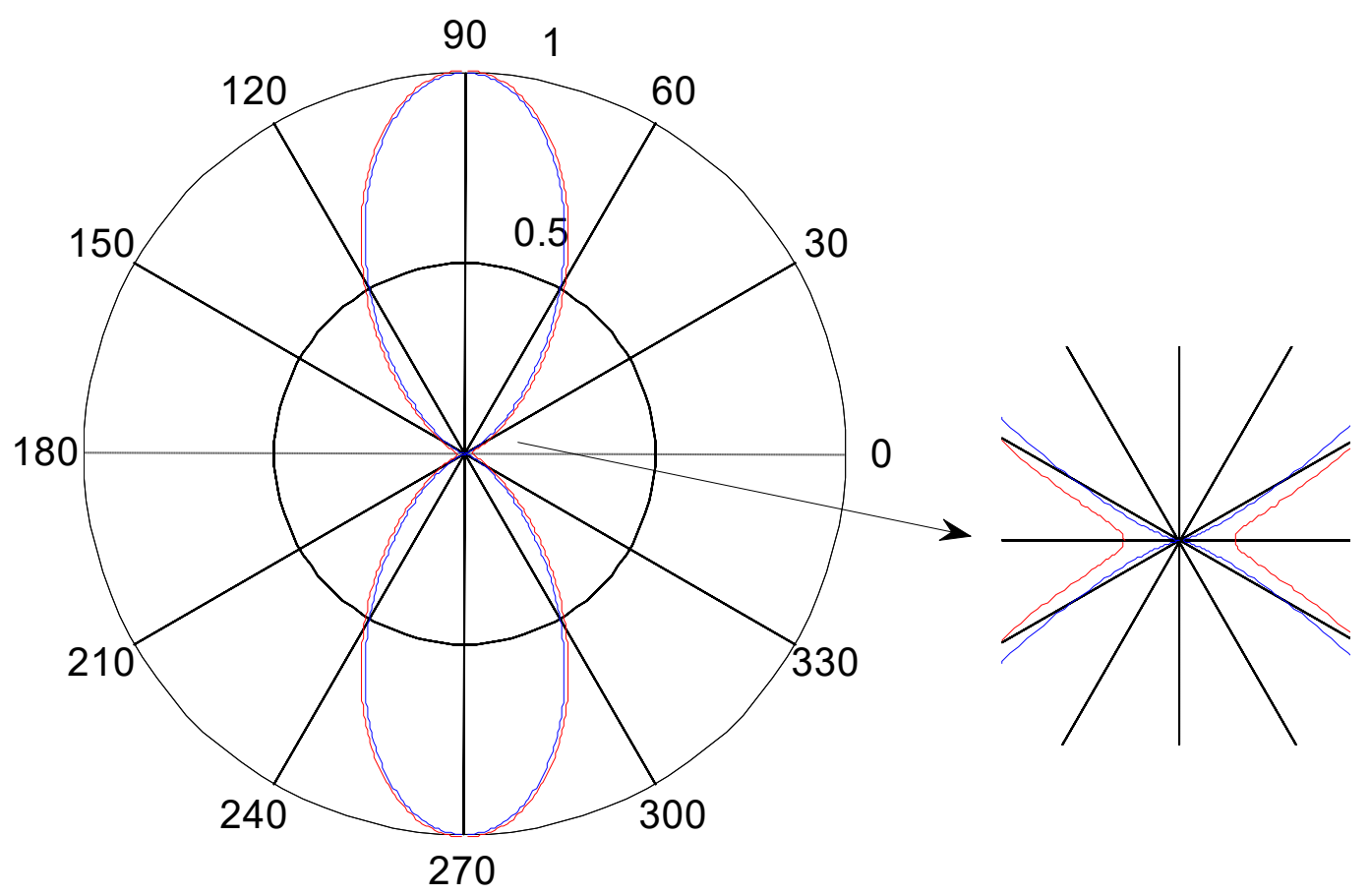

Figure 5.3 Radiation pattern: red for five cycles hamming windowed sine tone and blue for a single tone.

\subsection{Experimental Setup}

Figure 5.4 shows a diagram of the experimental setup used for the damage inspection. The experiment was carried out on an Al-2024 aluminum plate with dimensions of $480 \mathrm{~mm} \times$ $480 \mathrm{~mm} \times 3 \mathrm{~mm}$. Three circular PZT disks $(7 \mathrm{~mm}$ diameter, $0.2 \mathrm{~mm}$ thickness $)$ were permanently bonded on three vertices of an equilateral triangle with the dimensions of 7.5 $\mathrm{mm}$ which is equal to half the wavelength of a $100 \mathrm{kHz} \mathrm{A} 0$ mode Lamb wave. The triangle center was chosen to be the origin in all calculations. The experimental process has three steps. In each step, two of the PZT elements in the array serve as actuators, while the other serves as the sensor. Table 5.1 summarizes all three combinations of the transducers and Figure 5.5 shows the theoretical propagation pattern resulting from each of the three possible combinations in the experimental procedure. In each combination, two PZTs were 
excited simultaneously with a constant zero phase delay and the responses were recorded by the third PZT.

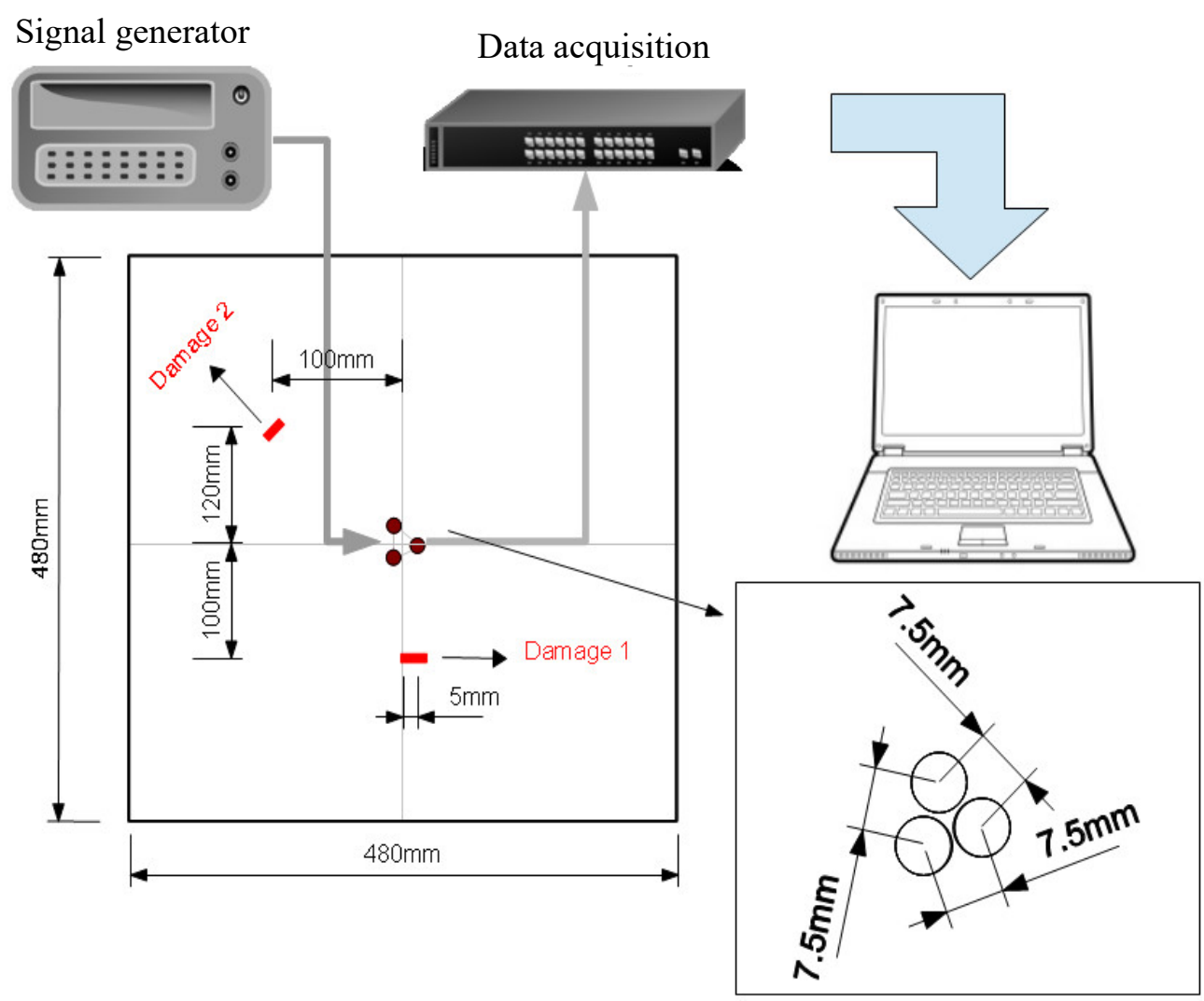

Figure 5.4 Experiment setup for the compact phased array approach.

Table 5.1 Different combinations in the experimental procedure

\begin{tabular}{c|ccc}
$\begin{array}{c}\text { COMBINATION } \\
\text { NUMBER }\end{array}$ & SOURCES & $\begin{array}{c}\text { PHASE } \\
\text { DIFFERENCES }\end{array}$ & $\begin{array}{c}\text { RECORD } \\
\text { FROM }\end{array}$ \\
\hline $\mathbf{1}$ & PZT 2 and PZT 3 & $0 \mathrm{deg}$ & PZT 1 \\
$\mathbf{2}$ & PZT 2 and PZT 1 & $0 \mathrm{deg}$ & PZT 3 \\
$\mathbf{3}$ & PZT 3 and PZT 1 & $0 \mathrm{deg}$ & PZT 2
\end{tabular}




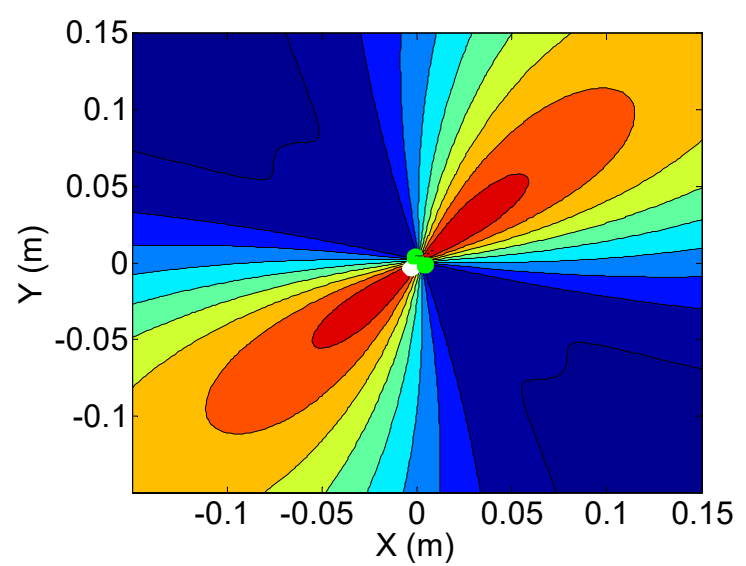

a)

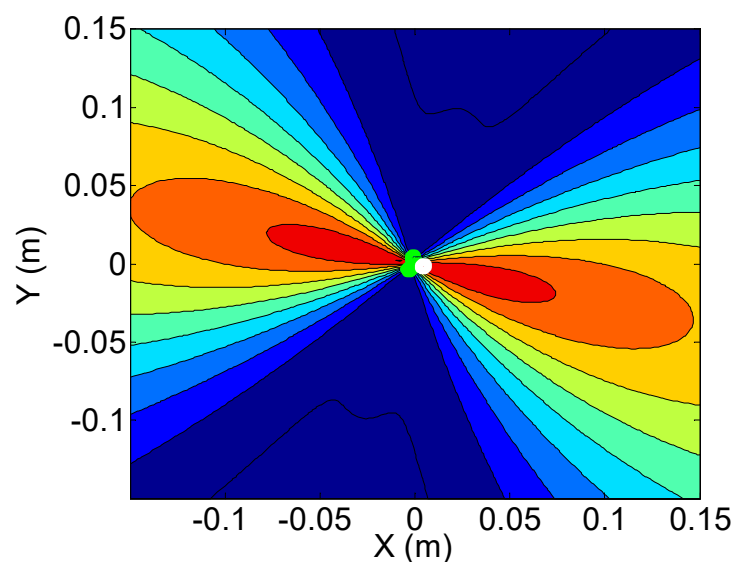

b)

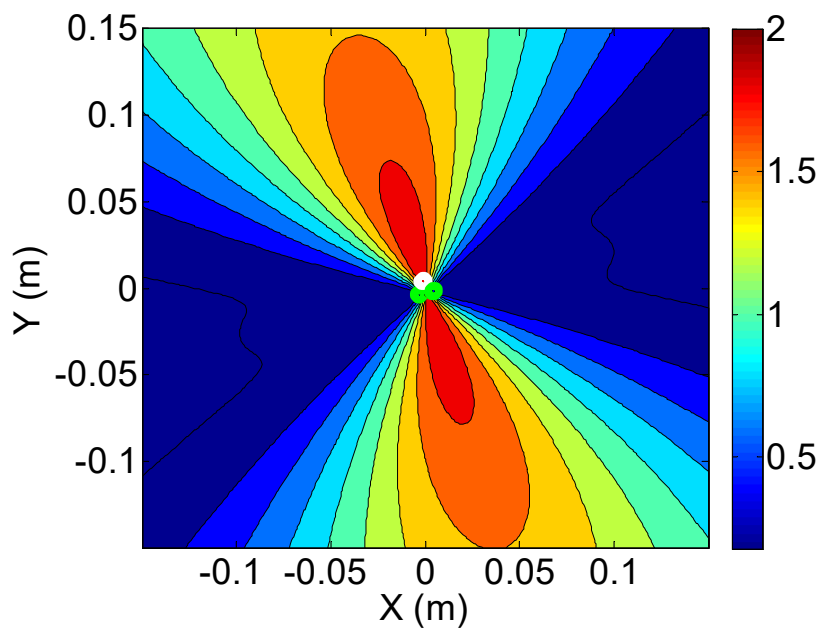

c)

Figure 5.5 Propagation pattern for different exciter combinations, green points show the actuators and the white ones show the receiver. a) Combination 1 b) Combination $2 \mathrm{c}$ ) Combination 3. 
Thus, the main lobes were directed in three different directions, without using additional complex electrical devices, and provide a complete coverage. A Rigol DG1022 arbitrary function generator was used for the excitation. A five cycle hamming windowed sine wave signal, with amplitude $20 \mathrm{Vpp}$ and center frequency of $100 \mathrm{kHz}$ was used as the excitation frequency. The $100 \mathrm{kHz}$ frequency was chosen since the antisymmetric mode A0 is dominant and $\mathrm{S} 0$ is weak, at this frequency. That is, the $\mathrm{A} 0 / \mathrm{S} 0$ strain ratio is approximately 3.63. Phase and group velocities were $1548 \mathrm{~m} / \mathrm{s}$ and $2620 \mathrm{~m} / \mathrm{s}$, respectively. Response signals were recorded by a DAQ unit at $2 \mathrm{MHz}$ sample rate. Figure 5.6 shows the test plate, PZTs, and the damage. Two sets of damage were created using a milling machine. The location and geometries of the them are shown in Table 5.2.

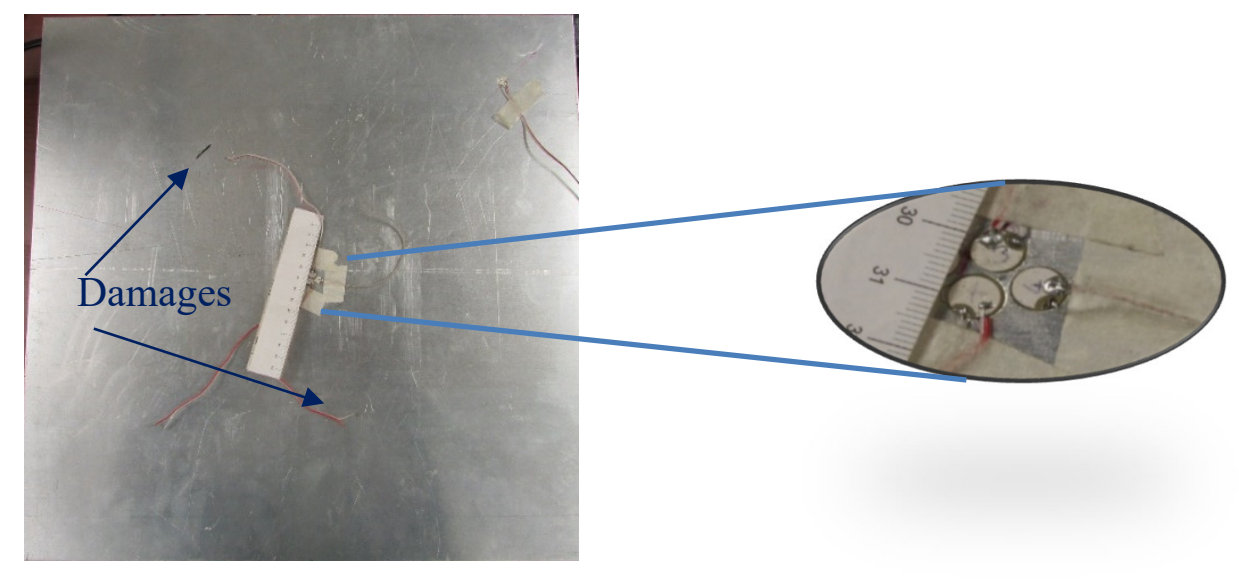

Figure 5.6 The test plate, PZTs, and damages.

Table 5.2 List of damages and their characteristics.

\begin{tabular}{|c|c|c|c|}
\hline Damage number & Cut size $(\mathrm{mm})$ & $\begin{array}{c}(x, y) \text { location of } \\
\text { damage center }\end{array}$ & $\begin{array}{c}\left(r, \Phi^{\circ}\right) \text { location of } \\
\text { damage center }\end{array}$ \\
\hline 1 & $10 * 2 * 3$ & $(5 \mathrm{~mm},-100 \mathrm{~mm})$ & $\left(100.12 \mathrm{~mm},-87.13^{\circ}\right)$. \\
\hline 2 & $10 * 2 * 3$ & $(-100 \mathrm{~mm}, 120 \mathrm{~mm})$ & $\left(156.2 \mathrm{~mm}, 150.2^{\circ}\right)$ \\
\hline
\end{tabular}




\subsection{Proposed Approach}

In the standard TFM, one exciter is used in each step. In this study, a revised TFM is proposed that is based on excitation of two transducers at the same time. In the standard approach, amplitudes of the reflected signals in each of the three excitation combinations are used for plotting a $2 \mathrm{D}$ image of the target surface as a function of position. For any point on the surface with the coordinates of $(\mathrm{x}, \mathrm{y}), d_{j}$ is the total propagation length and can be defined as follows:

$$
d_{j}=\sqrt{\left(x^{T}-x\right)^{2}+\left(y^{T}-y\right)^{2}}+\sqrt{\left(x^{R}-x\right)^{2}+\left(y^{R}-y\right)^{2}}
$$

where $\left(x^{T}, y^{T}\right)$ and $\left(\mathrm{x}^{\mathrm{R}}, \mathrm{y}^{\mathrm{R}}\right)$ are coordinates of the transmitters and the receiver, respectively. Subscript $j$ refers to the combination numbers which are shown in Table 5.1. In this study, two transducers are used in each excitation and the distance between transducers is insignificant, half a wave length. Therefore, the coordinates of the center of both transmitters were chosen to be equal to the coordinates of the origin in the calculations. As a result, the $d_{j}$ for each point on the target surface can be calculated as:

$$
d_{j}=\sqrt{x^{2}+y^{2}}+\sqrt{\left(x^{R}-x\right)^{2}+\left(y^{R}-y\right)^{2}}
$$

After excitation, the collected response is in the form of (time-voltage) graphs. $I_{j}(x, y)$ is the intensity of the reflection at each point. $I_{j}(x, y)$ can be calculated by obtaining the Hilbert transform of the difference of the received signals before $\left(a_{j}\right.$ base $\left.(t)\right)$ and after $\left(a_{j}(t)\right)$ appearance of a damage. In the following equation, $j$ is the number of combination set and $v_{g}$ is the group velocity of the antisymmetric mode at the selected excitation frequency. 


$$
I_{j}(x, y)=h_{j}\left(a_{j}\left(\frac{d_{j}}{v_{g}}\right)-a_{j_{\text {base }}}\left(\frac{d_{j}}{v_{g}}\right)\right)
$$

Because the excitation source is omni directional, the energy carried by the propagating waves in each direction decreases further from the excitation point. Therefore, the energy of the wave that is reflected from a closer point is larger from the energy of a wave reflected from a further point in the same direction. Considering this effect, the intensity can be adjusted to compensate for changes due to alteration of total path length, $d_{j}$. The standard intensity equation $I_{j}^{\prime}$, is shown in equation (5.7).

$$
I_{j}^{\prime}(x, y)=I_{j}(x, y) \sqrt{d_{j}(x, y)}
$$

To develop the modified approach, the image intensity information has to be updated with the wave interference data. Figure 5.7 shows the schematic of a two source array configuration used to calculate the intensity at a desired point on the surface.

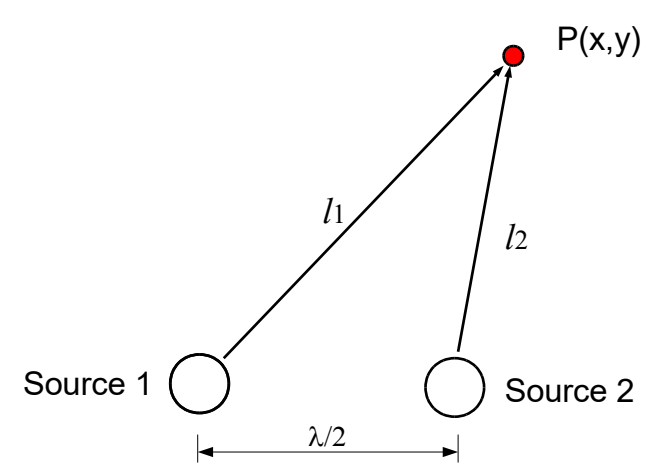

Figure 5.7 Two elements phased array.

The amplitude of the wave, resulting from interference of propagating waves from the two different sources at any point, $P$, can be calculated by using the phase difference $(\Delta \emptyset)$ between the two waves at that point. Therefore, the total amplitude at any point $(P)$ is equal 
to the summation of all frequency components effects, where $N$ is the number of frequency components.

$$
A_{j}(x, y)=\sum_{j=1}^{N} E(j)\left(1+\cos \left(\Delta \emptyset\left(j, v_{p}\right)\right)\right)
$$

By acquiring $A_{j}$ for different points on the plate, a theoretical 2D image of amplitudes on the plate can be obtained. The phase difference $(\Delta \emptyset)$ is a function of frequency and phase speed, and can be found by the following equation, where $\left(x_{1}, y_{1}\right)$ and $\left(x_{2}, y_{2}\right)$ are the coordinates of the exciter transducers.

$$
\Delta \emptyset\left(f, v_{p}\right)=\frac{\pi}{\lambda}\left(l_{1}-l_{2}\right)=\frac{\pi}{v_{p} / f}\left(\sqrt{\left(x_{1}-x\right)^{2}+\left(y_{1}-y\right)^{2}}-\sqrt{\left(x_{2}-x\right)^{2}+\left(y_{2}-y\right)^{2}}\right)
$$

In this study, it is suggested to use the multiplication of $I_{j}^{\prime}$ and $A_{j}$ (equation (5.10)) to obtain an accurate intensity. That is, the information of wave interference is applied to the intensity calculations in order to enhance the accuracy in the modified intensity.

$$
I_{j}^{\prime \prime}(x, y)=I_{j}^{\prime}(x, y) A_{j}(x, y)
$$

The simplified algorithm of the proposed method for the damage localization process for single and multiple damages is shown in Figure 5.8. The proposed approach can be used for detection of single and multiple damage. In presence of multiple defect, after detection of the first damage that area and its duplicate are subtracted from the total intensity $\left(I_{T}(x, y)\right)$. We refer to the subtraction process as masking in Figure 5.8. By masking the first damage and its effects, the method can find the next damage. The implementation of the proposed approach is discussed in details in the next section. 


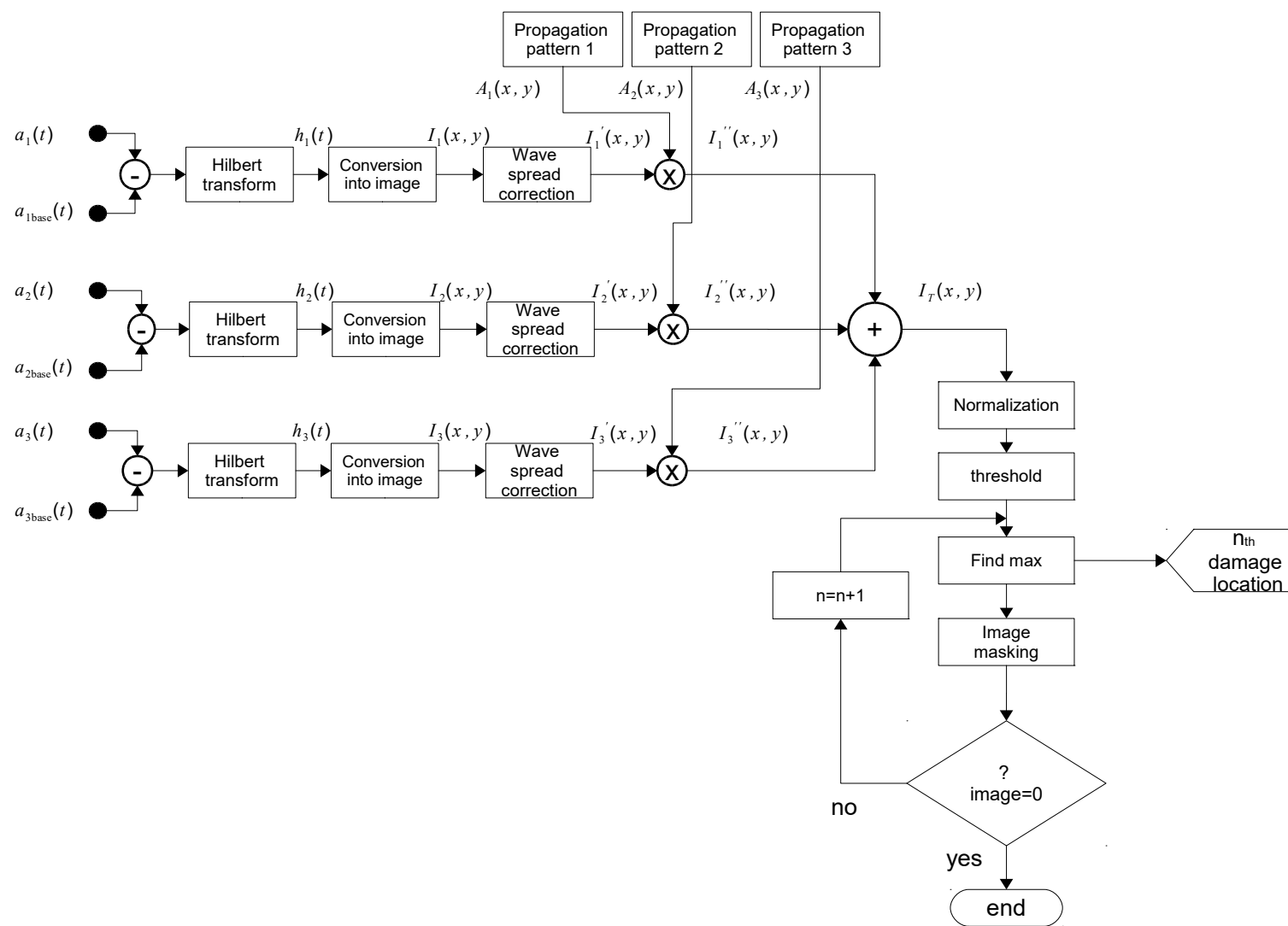

Figure 5.8 Simplified flow chart of the proposed method.

\subsection{Results and Discussion}

For detection of a single cut, damage 1 in Table 5.2 was created on the plate. Tests were conducted using the three different actuator and sensor combinations on the aluminum plate and are shown in Table 5.1. The intensity images resulting from standard and modified intensity formulas are compared to manifest the higher accuracy of the modified intensity approach in the localization of defects.

Figure 5.9, shows the 2D intensity image acquired from $I_{1}^{\prime}$ (standard approach) and $I_{1}^{\prime \prime}$ (modified approach) formulas for combinations 1-3, respectively. The dark red points indicate the most probable locations of the damage. 
Using equation (5.11) and (5.12), all intensity results shown in Figure 5.9 are summed and the normalized final intensity images from the standard and modified approaches are shown in Figure 5.10. Note that $\mathrm{n}$ indicates the number of combinations. In Figure 5.10 (a), the short black line shows the actual location of the damage. As can be seen from Figure 5.10 (a), the modified approach with interference correction significantly narrows down the defect area in the intensity map when compared to the intensity map resulting from the standard approach in Figure 5.10 (c). To obtain an improved image of the damaged area, the normalized intensity maps shown in Figure 5.10 (a) and Figure 5.10 (c) were filtered by applying a threshold equal to $97 \%$ of the max amplitude and the resultant images are shown in Figure 5.10 (b) and Figure 5.10 (d), respectively.

$$
\begin{aligned}
& I_{T}(x, y)=\sum_{j=1}^{n} I_{j}^{\prime \prime} \\
& I_{T}(x, y)=\sum_{j=1}^{n} I_{j}^{\prime}
\end{aligned}
$$

As can be seen from Figure 5.10 (b), the damage imaging algorithm detects two symmetrical damage locations, due to the symmetrical pattern of excitation. One of the locations is the actual estimated location of the damage and the other is simply a duplicate image of the estimated damage's location. It should be noted that the maximum intensities of these points are different and the location that has a higher intensity is the actual damage location. 


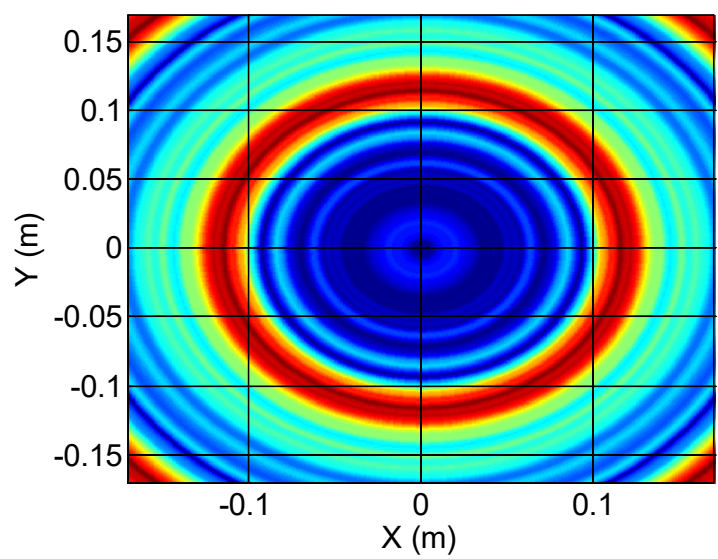

a) Combination 1 - standard approach

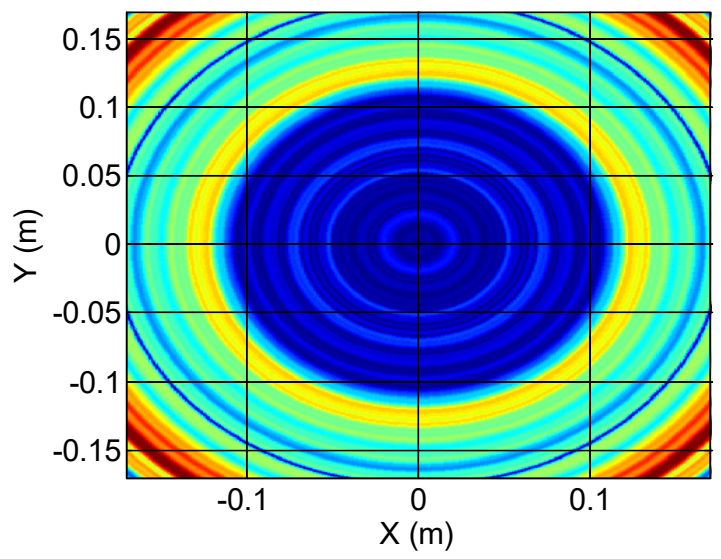

c) Combination 2 - standard approach

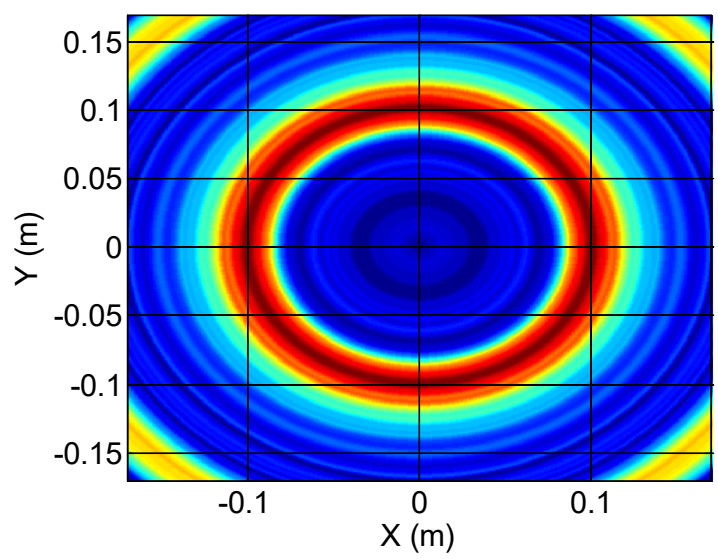

e) Combination 3 - standard approach

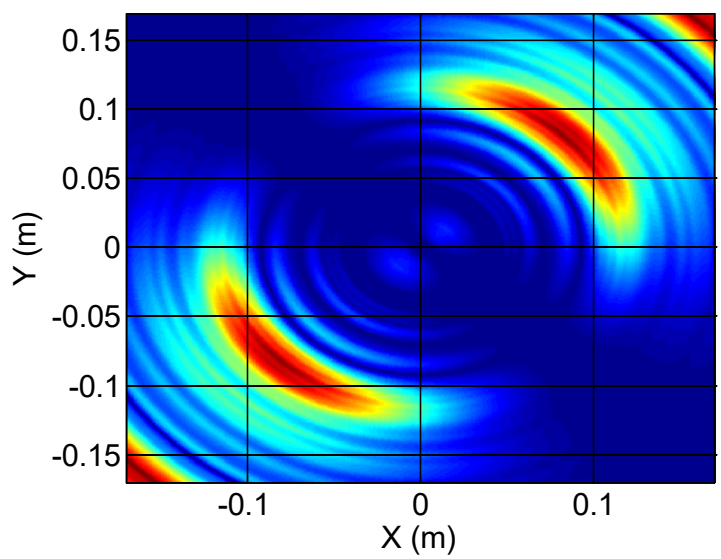

b) Combination 1- modified approach

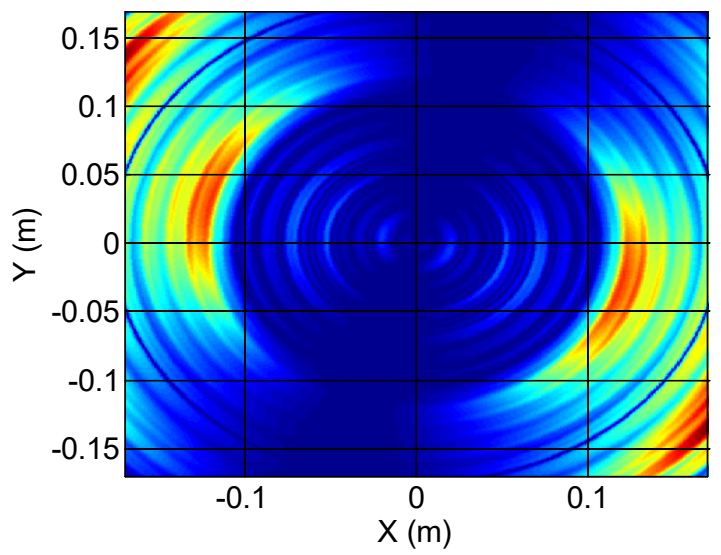

d) Combination 2 - modified approach

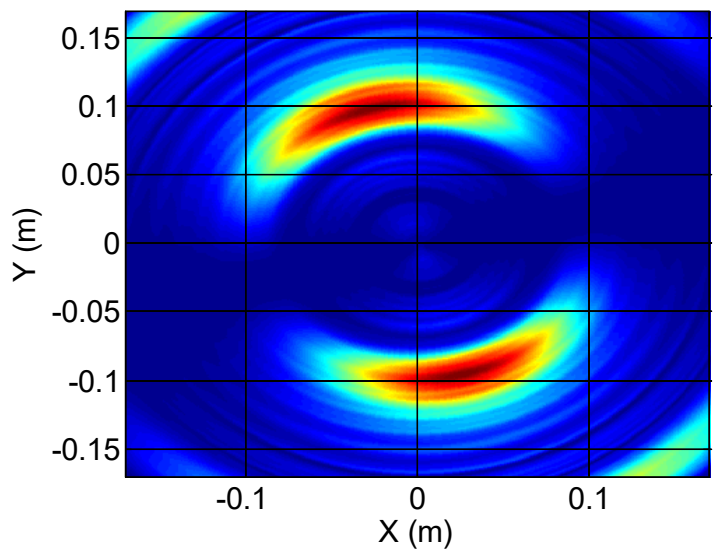

f) Combination 3 - modified approach

Figure 5.9 2D Intensity maps for test Combinations 1-3. 


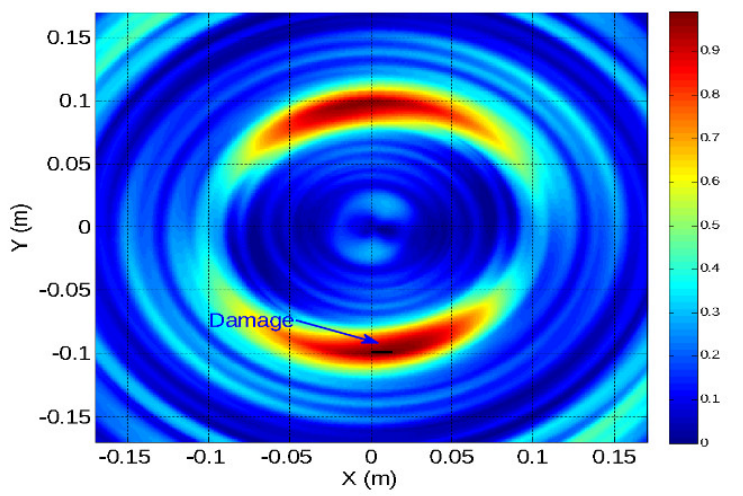

(a)

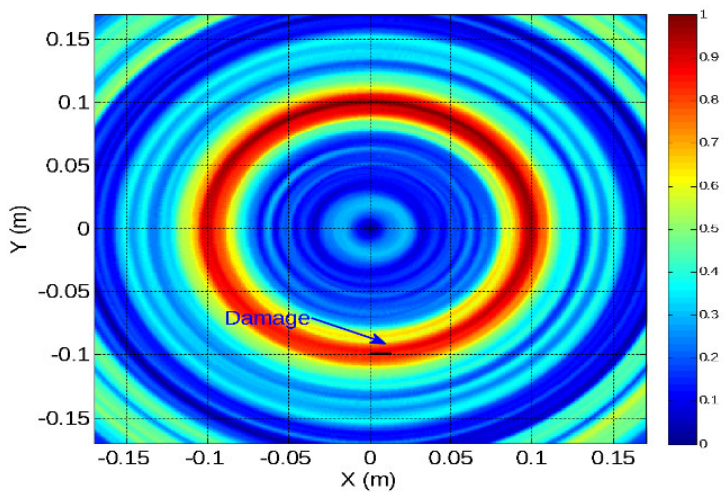

(c)

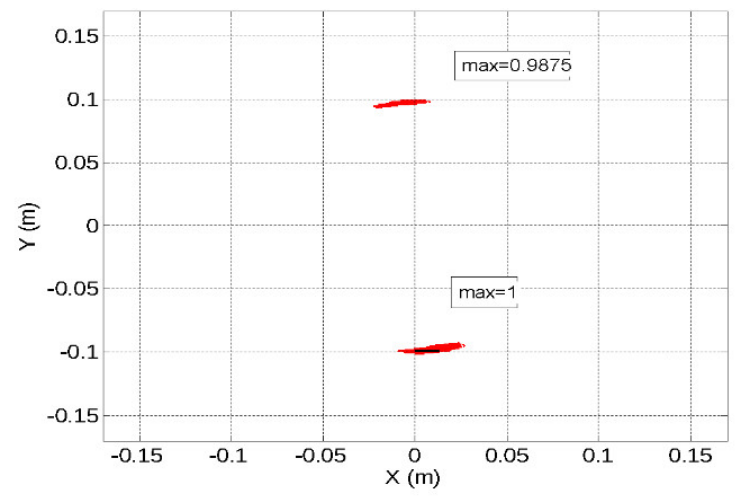

(b)

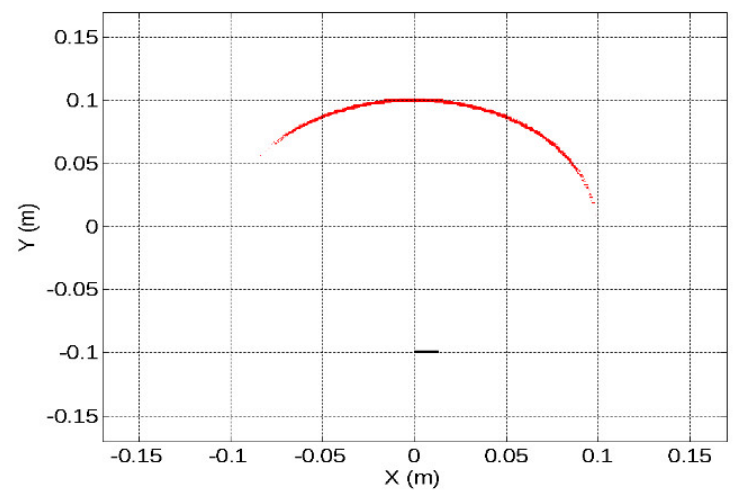

(d)

Figure 5.10 Total normalized intensity maps a) Intensity map resulted from the modified approach b) Intensity map of (a) after thresholding c) Intensity map resulted from standard approach d) Intensity map of (c) after thresholding.

In this test, the maximum intensity of the upper point reaches the value of 0.9875 while the maximum value of the lower point is 1 . The algorithm then sets the intensity around the duplicate location equal to zero. In Figure 5.11, the black area shows the actual location of the damage and the red area shows the calculated location of the damage using the modified approach after eliminating the duplicate of the estimated damage. 


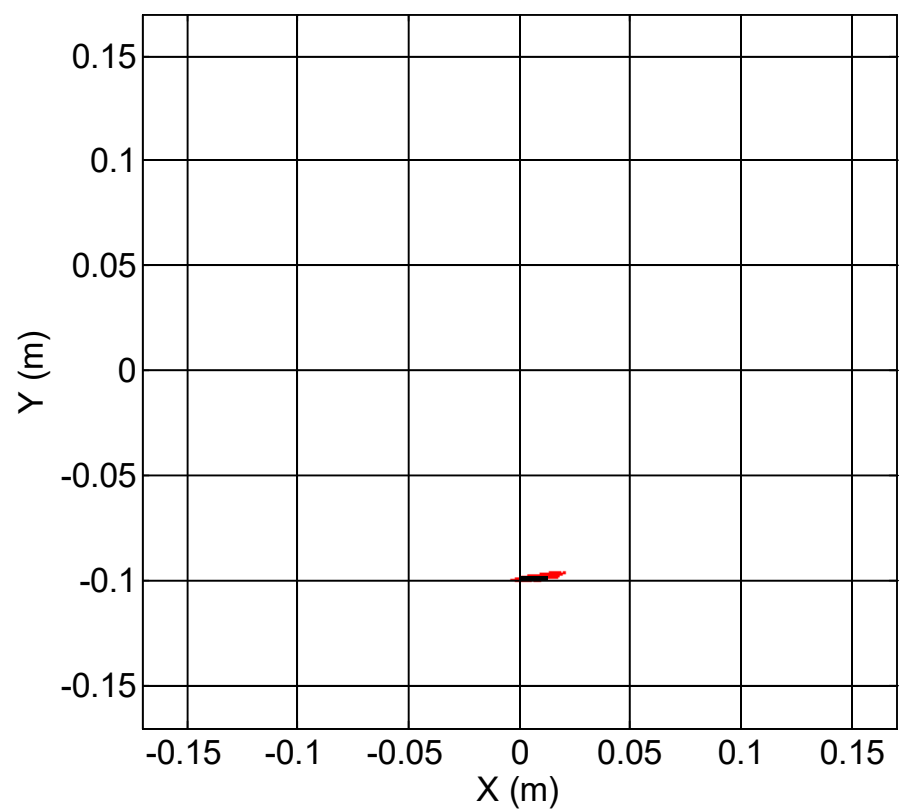

Figure 5.11 Estimated damage location (Black area shows real location of the damage).

To perform a detection test for multiple damage, in addition to damage one, a second damage was created and its location and geometry are tabulated in Table 5.2. The exact steps that were performed for detection of the single damage with modified approach were taken and after applying the same imaging technique, the first damage was identified. To find the second damage, the first damage had to be masked from the total intensity image. For that purpose, the max amplitude which indicates the first damage's location was found and then the damage and its duplicate regions were removed from the total intensity image. The second damage location was then determined with the same procedure. The total intensity image of multiple defects is shown in Figure 5.12 (a). The actual and estimated locations of multiple damage, after applying the $97 \%$ threshold and removing the duplicate points, are shown in Figure 5.12 (b). 


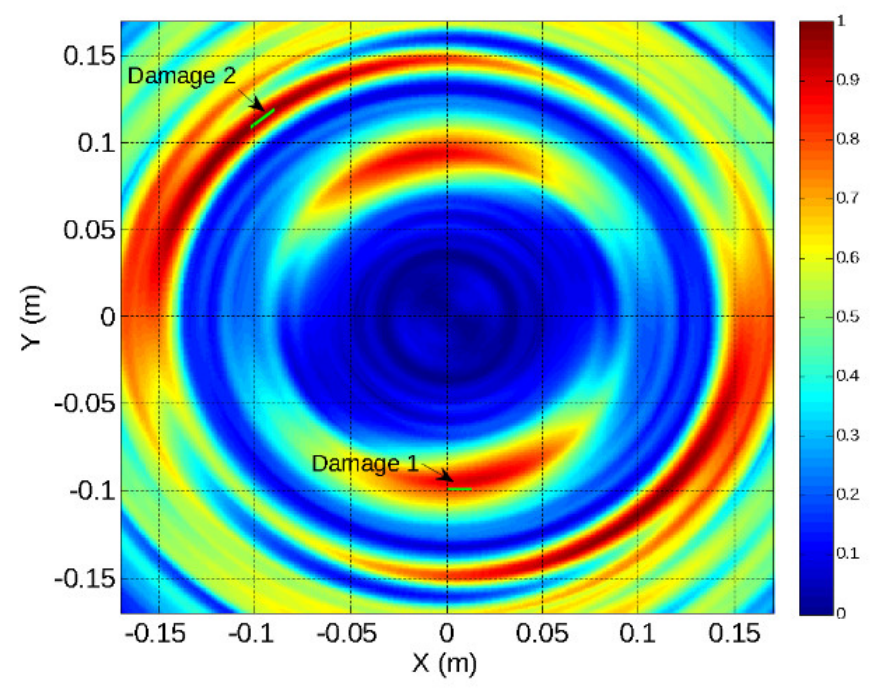

(a)

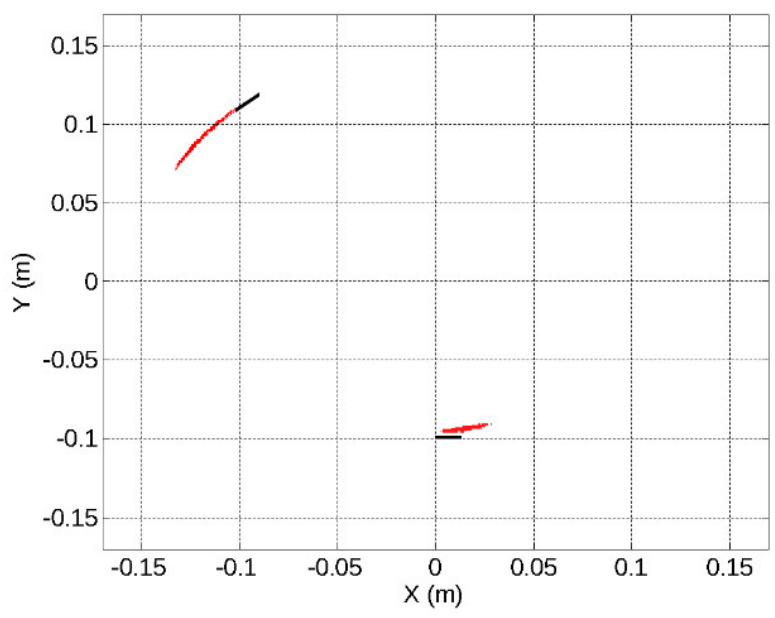

(b)

Figure 5.12 a) total normalized intensity map b) final estimated damage locations and actual damage locations.

The estimated locations of the first and second damages and the error in the radial distance and angular deviation of the estimated damage points from the actual locations are tabulated in Table 5.3. By using the compact phased array (CPA) method and choosing the appropriate excitation mode/frequency, the damages were located with high accuracy. The results indicate that the method can be used for detection of multiple defects with high accuracy. 
Table 5.3 Estimated location of multiple defects and associated errors in angular and radial directions.

\begin{tabular}{|c|c|c|c|c|c|c|}
\hline $\begin{array}{c}\text { Damage } \\
\text { number }\end{array}$ & $\begin{array}{c}\text { Estimated location } \\
(\mathrm{mm}, \text { degree })\end{array}$ & $\begin{array}{c}\text { Actual location } \\
(\mathrm{mm}, \text { degree })\end{array}$ & $\begin{array}{c}\text { Absolute difference } \\
\text { in radial direction }\end{array}$ & $\begin{array}{c}\text { Error in } \\
\text { radial } \\
\text { direction }\end{array}$ & $\begin{array}{c}\text { Absolute } \\
\text { angular } \\
\text { deviation }\end{array}$ & $\begin{array}{c}\text { Error in } \\
\text { angular } \\
\text { direction }\end{array}$ \\
\hline 1 & $\left(94.1,-81^{\circ}\right)$ & $\left(100.12,-87.13^{\circ}\right)$ & $6.02 \mathrm{~mm}$ & $6 \%$ & $6.13^{\circ}$ & $1.6 \%$ \\
\hline 2 & $\left(150.7,140^{\circ}\right)$ & $\left(156.2,150.2^{\circ}\right)$ & $5.5 \mathrm{~mm}$ & $3.5 \%$ & $10.2^{\circ}$ & $2.8 \%$ \\
\hline
\end{tabular}

\subsection{Summary}

Different types of transducer arrays and analysis algorithms have been used for localization of damages in structures. Phased array systems provide high SNR values in monitoring of the damage. However, pulse or phase shifters, multiplexers and a high number of transducers are associated with phased array systems of transducers. This study proposes a compact phased array (CPA) method for three step imaging of multiple damages.

In this study, three PZT transducers in a phased array system were mounted on an aluminum plate. The distances between the transducers were chosen to be equal to half the wavelength of the central excitation frequency. Three different combinations of actuator and sensor configurations were utilized for complete scanning of the surface. Each time, two transducers served as actuators and the third transducer collected the response. The wave propagation and interference information in each combination was included to improve the results of the total focusing method in the analysis. The CPA was able to successfully locate single and multiple cracks with high accuracy.

The proposed method doesn't need to apply high voltages to maintain acceptable SNRs. Instead, the CPA provides complete coverage by creating beams in desired directions in 
three increments. The low excitation voltage, the small number of transducers, and short post processing time along with high localization accuracy demonstrates that the proposed method has great potential for in-situ monitoring of critical structures. 


\section{CHAPTER 6}

\section{Conclusions and Future Directions}

\subsection{Conclusions}

Structural health monitoring approaches help to maximize the life of in-service structures and to minimize catastrophic failures. By considering the performance of current inspection methods, development of more accurate and cost-effective SHM techniques for online monitoring of structures seems to be a must for maintaining the safety of aging structures.

In practice, there is no or limited a priori knowledge about the size, shape, and orientation of a defect in an in-service structure. Characteristics of a wave, such as the wave mode, frequency, dispersion and propagation characteristics, determine the types of defects that are detectable through excitation of that mode. In short, the performance of the conventional methods depends on the match between the selected excitation mode and geometrical parameters of the damage that is yet to be detected, which makes the whole enterprise of detecting structural defects logically circular.

This study used novel linear and nonlinear methods using single and broad frequency excitations to minimize the problems associated with detection and localization of defects. The main achievements and results are summarized as follows:

1) The surface response to excitation method was originally developed for detection of defects and monitoring of loads applied on plate-like structures. The main benefits of the SuRE method include the lack of the need for EMI analyzers and its 
higher sensitivity to defects not in close proximity of the PZT element. In this study, the validity of surface response to excitation method is discussed theoretically and the method is implemented experimentally for detection of both internal and external damages in pipes.

2) In some applications, because of design considerations and high temperature, sensors cannot be attached to the surface of the structure. Therefore, the SuRE method was used to develop a non-contact strategy for detection of defects in pipes with minimum dependency on the selection of proper excitation modes and frequencies.

3) It was observed that the SuRE method can detect different types of internal and external damages, in contact and non-contact modes, in pipes. Also, the SuRE method might be able to quantify between different severities of damages. The study showed that the SuRE method can be used to acquire quick and reliable information about the state of the health of pipes.

4) The SSD index was used as the damage index in the SuRE method. The damage index showed negligible sensitivity to changes in boundary conditions while it maintained a high sensitivity to defects with different characteristics at all sensor locations.

5) Certain types of damages, such as debondings, imperfect contacts between mating surfaces, and cracks, produce nonlinear effects in the response of structures to bitonal excitations. Nonlinear wave modulation spectroscopy (NWMS) (vibroacoustic modulation) detects the defects by tracking the development of nonlinear characteristics in the early stages of their development. In this approach, two 
different actuators are used to excite the structure with two distinct frequencies. Selection of these frequencies plays an important role in the successful detection of defects. To date, no convenient and systematic approach has been developed for proper selection of the two initial excitation frequencies in a single test. In this study, we proposed the use of two sweep sine waves covering broad ranges of frequencies to address this issue.

6) Use of broad band frequencies eliminated the need for selection of distinctive pumping and probing frequencies. We referred to this new method as Comprehensive Heterodyning Effect Based Inspection (CHEBI).

7) In this study, the CHEBI method was implemented for the detection of cracks. Unlike previously available methods, using the CHEBI method, it is possible to analyze the nonlinear behavior of a structure in multiple and higher ranges of frequency combinations in one single test. The method doesn't require one of the frequencies to be very low.

8) Application of broad frequency ranges with ascending and descending sweep excitations allows the method to be sensitive to various types of potential defects. This new approach may be easily applied for the detection of different types of defects without significant modifications. The results showed that the double sweep sine wave excitations can be effectively used for detecting cracks of varying lengths. The method may be used in different industries for quick and reliable assessment of structures without requiring baseline data.

9) In this study, the very first sensor-free structural health monitoring (SSHM) system was introduced to propose a low-cost alternative to SHM systems. The SSHM 
system was used for detecting the loose bolts in two plates and a washer system. Beep sounds were produced at the location of loose bolts. Instead of beep sounds, personal messages can also be used to create meaningful verbal warnings. The proposed SSHM approach can be used independently or can be conveniently integrated into the conventional active SHM systems as a backup module.

10) In this study, a compact phased array (CPA) system was developed for localization of single and multiple defects in an aluminum plate. The CPA used three piezoelectric elements. The wave propagation information was included in the updated total focusing method (TFM) for improving the accuracy in the localization of defects. Results indicated that the CPA approach can locate single and multiple defects with high accuracy while decreasing the processing costs and number of required transducers.

\subsection{Limitations and Future Work}

This study used broad band excitation in linear and nonlinear monitoring processes to minimize the dependency on a priori knowledge about characteristics of potential defects in the detection processes. Also, a compact phased array method was developed for locating defects with only three transducers. Here, the following suggestions for future studies in these areas are proposed.

1) It was shown that the SuRE method can detect the damages in all sensing points. The future work regarding the SuRE method should focus on the selection of optimal sensing points for increasing the sensitivity of the monitoring process for quantification purposes. 
2) To date, in the SuRE method, the actuator element has been a bonded PZT element. The efficiency of the method using non-contact excitation should be studied.

3) The CHEBI method was implemented for comprehensive detection of cracks and loose bolts. The method can be applied for detecting other types of defects, such as debonding, delamination, and other damages that produce nonlinear effects.

4) The sensor-less approach was tested successfully for detecting loose bolts in plates and washer systems. With slight variations, the method has the potential to be used for sensor-free detection of other types of damages, which manifest similar nonlinear behavior.

5) Instead of beep sounds in the SSHM system, personal messages or special signals can be designed to be only sensitive to certain types of defects.

6) To date, locating defects is mainly carried out by linear methods. Future investigations should be focused on localization of defects using nonlinear methods in transducer array systems. 


\section{Bibliography}

[1] V. Giurgiutiu, A. Zagrai, and J. Jing Bao, "Piezoelectric Wafer Embedded Active Sensors for Aging Aircraft Structural Health Monitoring," Struct. Heal. Monit., vol. 1, no. 1, pp. 41-61, Jul. 2002.

[2] M. Sun, W. J. Staszewski, and R. N. Swamy, "Smart sensing technologies for structural health monitoring of civil engineering structures," Adv. Civ. Eng., vol. $2010,2010$.

[3] P. C. Chang, A. Flatau, and S. C. Liu, "Review paper: Health monitoring of civil infrastructure," Struct. Heal. Monit., vol. 2, no. 3, pp. 257-267, 2003.

[4] C. I. Ossai, B. Boswell, and I. J. Davies, "Pipeline failures in corrosive environments - A conceptual analysis of trends and effects," Eng. Fail. Anal., vol. 53, no. May, pp. 36-58, 2015.

[5] ASCE, “A comprehensive assessment of America's Infrastructure,” 2017.

[6] H. Sohn, H. J. Lim, M. P. Desimio, K. Brown, and M. Derriso, "Nonlinear ultrasonic wave modulation for online fatigue crack detection," J. Sound Vib., vol. 333, no. 5, pp. 1473-1484, 2014.

[7] ASCE, "Infrastructure report card," ASCE News, vol. 53, no. September 2013, pp. $1-36,2016$.

[8] D. of T. P. and H. M. S. Administration, "PHMSA - Data \&amp; Statistics."

[9] K. B. Misra(Editor), Handbook of Performability Engineering, no. August 2008. London: Springer London, 2008.

[10] L. Swanson, "Linking maintenance strategies to performance," Int. J. Prod. Econ., vol. 70, pp. 237-244, 2001. 
[11] I. Alsyouf, "The role of maintenance in improving companies' productivity and profitability," Int. J. Prod. Econ., vol. 105, no. 1, pp. 70-78, 2007.

[12] Y. Liu, S. Mohanty, and A. Chattopadhyay, "Condition based structural health monitoring and prognosis of composite structures under uniaxial and biaxial loading," J. Nondestruct. Eval., vol. 29, no. 3, pp. 181-188, 2010.

[13] A. K. S. Jardine, D. Lin, and D. Banjevic, "A review on machinery diagnostics and prognostics implementing condition-based maintenance," Mech. Syst. Signal Process., vol. 20, no. 7, pp. 1483-1510, 2006.

[14] T. Nagayama et al., "Structural Health Monitoring Using Smart Sensors," 2008.

[15] P. Shull, Nondestructive evaluation: theory, techniques, and applications. CRC press, 2016.

[16] C. R. Farrar and K. Worden, "An introduction to structural health monitoring," Philos. Trans. R. Soc. A Math. Phys. Eng. Sci., vol. 365, no. 1851, pp. 303-315, 2007.

[17] S. Deutsch, "A preliminary study of the fluid mechanics of liquid penetrant testing," J. Res. Natl. Bur. Stand., 1979.

[18] C. Farrar and S. Doebling, "An overview of modal-based damage identification methods," Proc. DAMAS, 1997.

[19] H. Nishino, S. Takashina, F. Uchida, M. Takemoto, and K. Ono, "Modal analysis of hollow cylindrical guided waves and applications," Japanese J. Appl. Physics, Part 1 Regul. Pap. Short Notes Rev. Pap., vol. 40, no. 1, pp. 364-370, 2001.

[20] C. Chen, Ultrasonic and advanced methods for nondestructive testing and material characterization. World Scientific Publishing Co Pte Ltd, 2007.

[21] C. Meola and G. M. Carlomagno, "Recent advances in the use of infrared thermography," Meas. Sci. Technol., vol. 15, no. 9, pp. R27-R58, 2004. 
[22] M. Pan, Y. He, G. Tian, D. Chen, and F. Luo, "Defect characterisation using pulsed eddy current thermography under transmission mode and NDT applications," NDT E Int., vol. 52, pp. 28-36, 2012.

[23] W. B. Gilboy, "X- and $\gamma$-ray tomography in NDE applications," Nucl. Instruments Methods Phys. Res., vol. 221, no. 1, pp. 193-200, Mar. 1984.

[24] L. Cheng and G. Y. Tian, "Comparison of nondestructive testing methods on detection of delaminations in composites," J. Sensors, vol. 2012, 2012.

[25] 1977- Kessler, Seth Stovack, "Piezoelectric-based in-situ damage detection of composite materials for structural health monitoring systems," Massachusetts Institute of Technology, 2002.

[26] S. Elliot, "DevOps and the Cost of Downtime: Fortune 1000 Best Practice Metrics Quantified IDC OPINION," 2014.

[27] C. R. Farrar and K. Worden, "An introduction to structural health monitoring," Philos. Trans. R. Soc. A Math. Phys. Eng. Sci., vol. 365, no. 1851, pp. 303-315, Feb. 2007.

[28] W. J. Staszewski, S. Mahzan, and R. Traynor, "Health monitoring of aerospace composite structures - Active and passive approach," Compos. Sci. Technol., vol. 69, no. 11-12, pp. 1678-1685, 2009.

[29] M. Demetgul, V. Y. Senyurek, R. Uyandik, I. N. Tansel, and O. Yazicioglu, "Evaluation of the health of riveted joints with active and passive structural health monitoring techniques," Measurement, vol. 69, pp. 42-51, 2015.

[30] X. Li, "A brief review: Acoustic emission method for tool wear monitoring during turning," Int. J. Mach. Tools Manuf., vol. 42, no. 2, pp. 157-165, 2002.

[31] X. Zhao et al., "Active health monitoring of an aircraft wing with an embedded piezoelectric sensor/actuator network: II. Wireless approaches,” Smart Mater. Struct., vol. 16, no. 4, pp. 1218-1225, 2007. 
[32] J. L. Rose and P. B. Nagy, "Ultrasonic Waves in Solid Media," J. Acoust. Soc. Am., vol. 107, no. 4, pp. 1807-1808, Apr. 2000.

[33] Y. Lu, L. Ye, D. Wang, L. Zhou, and L. Cheng, "Piezo-activated guided wave propagation and interaction with damage in tubular structures," Smart Struct. Syst., vol. 6, no. 7, pp. 835-849, Sep. 2010.

[34] D. N. Alleyne, B. Pavlakovic, M. J. S. Lowe, and P. Cawley, "Rapid, Long Range Inspection of Chemical Plant Pipework Using Guided Waves," Key Eng. Mater., vol. 270-273, pp. 434-441, 2004.

[35] D. Alleyne, M. Lowe, and P. Cawley, "The Inspection of Chemical Plant Pipework Using Lamb Waves: Defect Sensitivity and Field Experience," in Review of Progress in Quantitative Nondestructive Evaluation, Boston, MA: Springer US, 1996, pp. 1859-1866.

[36] A. Arnau, Piezoelectric Transducers and Applications. Berlin, Heidelberg: Springer Berlin Heidelberg, 2004.

[37] P. Wilcox, M. Lowe, and P. Cawley, "The effect of dispersion on long-range inspection using ultrasonic guided waves," NDT E Int., vol. 34, no. 1, pp. 1-9, 2001.

[38] W. Q. Chen, H. M. Wang, and R. H. Bao, "On calculating dispersion curves of waves in a functionally graded elastic plate," Compos. Struct., vol. 81, no. 2, pp. 233-242, 2007.

[39] T. Hayashi, W. J. Song, and J. L. Rose, "Guided wave dispersion curves for a bar with an arbitrary cross-section, a rod and rail example," Ultrasonics, vol. 41, no. 3 , pp. 175-183, 2003.

[40] M. Gresil and V. Giurgiutiu, "Time-Domain Hybrid Global-Local Prediction of Guided Waves Interaction with Damage," Key Eng. Mater., vol. 558, no. JUNE, pp. 116-127, 2013. 
[41] V. Giurgiutiu, "Tuned Lamb Wave Excitation and Detection with Piezoelectric Wafer Active Sensors for Structural Health Monitoring," J. Intell. Mater. Syst. Struct., vol. 16, no. 4, pp. 291-305, 2005.

[42] B. Xu and V. Giurgiutiu, "Single Mode Tuning Effects on Lamb Wave Time Reversal with Piezoelectric Wafer Active Sensors for Structural Health Monitoring," J. Nondestruct. Eval., vol. 26, no. 2-4, pp. 123-134, Nov. 2007.

[43] H. Sohn and S. J. Lee, "Lamb wave tuning curve calibration for surface-bonded piezoelectric transducers," Smart Mater. Struct., vol. 19, no. 1, p. 15007, 2010.

[44] M. Gresil, L. Yu, V. Giurgiutiu, and M. Sutton, "Predictive modeling of electromechanical impedance spectroscopy for composite materials," Struct. Heal. Monit., vol. 11, no. 6, pp. 671-683, 2012.

[45] J. E. Michaels, S. J. Lee, A. J. Croxford, and P. D. Wilcox, "Chirp excitation of ultrasonic guided waves," Ultrasonics, vol. 53, no. 1, pp. 265-270, 2013.

[46] Venu Gopal Madhav Annamdas and Chee Kiong Soh, "Application of Electromechanical Impedance Technique for Engineering Structures: Review and Future Issues," J. Intell. Mater. Syst. Struct., vol. 21, no. 1, pp. 41-59, 2010.

[47] T. G. S. Overly et al., "Development of an extremely compact impedance-based wireless sensing device," Smart Mater. Struct., vol. 17, no. 6, p. 65011, Dec. 2008.

[48] S. Park, B. L. Grisso, D. J. Inman, and C.-B. Yun, "MFC-Based Structural Health Monitoring Using a Miniaturized Impedance Measuring Chip for Corrosion Detection," Res. Nondestruct. Eval., vol. 18, no. 2, pp. 139-150, Apr. 2007.

[49] C. Bermes, J. Kim, J. Qu, and L. J. Jacobs, "Nonlinear Lamb waves for the detection of material nonlinearity," Mech. Syst. Signal Process., vol. 22, no. 3, pp. 638-646, Apr. 2008. 
[50] H. Miao, Q. Huan, Q. Wang, and F. Li, "Excitation and reception of single torsional wave $\mathrm{T}(0,1)$ mode in pipes using face-shear $\mathrm{d} 24$ piezoelectric ring array,” Smart Mater. Struct., vol. 26, no. 2, p. 25021, Feb. 2017.

[51] S. Delrue, M. Tabatabaeipour, J. Hettler, and K. Van Den Abeele, “Applying a nonlinear, pitch-catch, ultrasonic technique for the detection of kissing bonds in friction stir welds," Ultrasonics, vol. 68, pp. 71-79, 2016.

[52] J. Hettler, M. Tabatabaeipour, S. Delrue, and K. Van Den Abeele, "Linear and nonlinear Guided Wave imaging of impact damage in CFRP using a probabilistic approach,” Materials (Basel)., vol. 9, no. 11, pp. 1-17, 2016.

[53] D. Broda, W. J. Staszewski, A. Martowicz, T. Uhl, and V. V. Silberschmidt, "Modelling of nonlinear crack-wave interactions for damage detection based on ultrasound-A review," J. Sound Vib., vol. 333, no. 4, pp. 1097-1118, Feb. 2014.

[54] Y. F. Shen and V. Giurgiutiu, "Predictive modeling of nonlinear wave propagation for structural health monitoring with piezoelectric wafer active sensors," J. Intell. Mater. Syst. Struct., vol. 25, no. 4, pp. 506-520, 2014.

[55] Y. Shen and V. Giurgiutiu, "Predictive modeling of nonlinear wave propagation for structural health monitoring with piezoelectric wafer active sensors," J. Intell. Mater. Syst. Struct., vol. 25, no. 4, pp. 506-520, 2014.

[56] H. Lee and H. Sohn, "Damage detection for pipeline structures using optic-based active sensing,” Smart Struct. Syst., vol. 9, no. 5, pp. 461-472, May 2012.

[57] W.-B. Na and H.-S. Yoon, "Parametric density concept for long-range pipeline health monitoring," Smart Struct. Syst., vol. 3, no. 3, pp. 357-372, Jul. 2007.

[58] J. L. Rose†, "Dispersion Curves in Guided Wave Testing," Mater. Eval., vol. 61, no. 1, 2003. 
[59] H. Nishino, K. Yoshida, H. Cho, and M. Takemoto, "Propagation phenomena of wideband guided waves in a bended pipe," Ultrasonics, vol. 44, pp. e1139-e1143, 2006.

[60] W. Zhou, F. G. Yuan, and T. Shi, "Guided torsional wave generation of a linear inplane shear piezoelectric array in metallic pipes," Ultrasonics, vol. 65, pp. 69-77, 2016.

[61] X. Wang, P. W. Tse, C. K. Mechefske, and M. Hua, "Experimental investigation of reflection in guided wave-based inspection for the characterization of pipeline defects," NDT E Int., vol. 43, no. 4, pp. 365-374, 2010.

[62] A. Demma, P. Cawley, M. Lowe, A. G. Roosenbrand, and B. Pavlakovic, "The reflection of guided waves from notches in pipes: A guide for interpreting corrosion measurements," NDT E Int., vol. 37, no. 3, pp. 167-180, 2004.

[63] V. Giurgiutiu, M. Gresil, B. Lin, A. Cuc, Y. Shen, and C. Roman, "Predictive modeling of piezoelectric wafer active sensors interaction with high-frequency structural waves and vibration," Acta Mech., vol. 223, no. 8, pp. 1681-1691, 2012.

[64] S. Park et al., "PZT-based active damage detection techniques for steel bridge components," Smart Mater. Struct., vol. 15, no. 4, pp. 957-966, Aug. 2006.

[65] Z. Su, X. Wang, Z. Chen, L. Ye, and D. Wang, "A built-in Active Sensor Network for Health Monitoring of Composite Structures," Smart Mater. Struct., vol. 15, pp. 1939-1949, 2006.

[66] R. Carandente and P. Cawley, "The effect of complex defect profiles on the reflection of the fundamental torsional mode in pipes," NDT E Int., vol. 46, no. 1, pp. 41-47, 2012.

[67] S. Ma, Z. Wu, Y. Wang, and K. Liu, "The reflection of guided waves from simple dents in pipes," Ultrasonics, vol. 57, no. C, pp. 190-197, 2015. 
[68] H. W. Kim, H. J. Lee, and Y. Y. Kim, "Health monitoring of axially-cracked pipes by using helically propagating shear-horizontal waves," NDT E Int., vol. 46, no. 1, pp. 115-121, 2012.

[69] J.-H. Lee and S.-J. Lee, “Application of laser-generated guided wave for evaluation of corrosion in carbon steel pipe," NDT E Int., vol. 42, no. 3, pp. 222227, 2009.

[70] V. G. Annamdas and M. a. Radhika, "Electromechanical impedance of piezoelectric transducers for monitoring metallic and non-metallic structures: A review of wired, wireless and energy-harvesting methods," J. Intell. Mater. Syst. Struct., vol. 24, no. 9, pp. 1021-1042, 2013.

[71] G. Park, H. Sohn, C. R. Farrar, and D. J. Inman, “Overview of Piezoelectric Impedance-Based Health Monitoring and Path Forward," Shock Vib. Dig., vol. 35, no. 6, pp. 451-463, 2003.

[72] S. Park, C. C. B. Yun, Y. Roh, and J. Lee, "Health monitoring of steel structures using impedance of thickness modes at PZT patches," SMART Struct. Syst., vol. 1, pp. 339-353, 2005.

[73] H. Fekrmandi et al., "Implementation of the Surface Response to Excitation (SuRE) Method with DSP's for Detection of the Damage of Thick Blocks," in Structural Health Monitoring 2015, 2015.

[74] S. Tashakori et al., "Load Monitoring Using Surface Response to Excitation Method," Springer International Publishing, 2017, pp. 209-214.

[75] H. Fekrmandi et al., "A non-contact method for part-based process performance monitoring in end milling operations," Int. J. Adv. Manuf. Technol., vol. 83, no. 14, pp. 13-20, Mar. 2016. 
[76] S. Tashakori et al., "Contact and non-contact approaches in load monitoring applications using surface response to excitation method," Measurement, vol. 89, pp. 197-203, Jul. 2016.

[77] C. Y. K. Chee, L. Tong, and G. P. Steven, "A Review on the Modelling of Piezoelectric Sensors and Actuators Incorporated in Intelligent Structures," $J$. Intell. Mater. Syst. Struct., vol. 9, no. 1, pp. 3-19, Jan. 1998.

[78] A. Preumont, Vibration Control of Active Structures, vol. 179. Dordrecht: Springer Netherlands, 2011.

[79] W. Zhou, F.-G. Yuan, and T. Shi, "Guided torsional wave generation of a linear in-plane shear piezoelectric array in metallic pipes," Ultrasonics, vol. 65, pp. 6977, 2016.

[80] F. Li, Z. Liu, X. Sun, H. Li, and G. Meng, "Propagation of guided waves in pressure vessel,” Wave Motion, vol. 52, pp. 216-228, 2015.

[81] K. Diamanti and C. Soutis, "Structural health monitoring techniques for aircraft composite structures," Prog. Aerosp. Sci., vol. 46, no. 8, pp. 342-352, 2010.

[82] M. Carboni, A. Gianneo, and M. Giglio, "A Lamb waves based statistical approach to structural health monitoring of carbon fibre reinforced polymer composites," Ultrasonics, vol. 60, pp. 51-64, 2015.

[83] A. Baltazar, E. Rojas, and R. Mijarez, "Structural Health Monitoring in Cylindrical Structures Using Helical Guided Wave Propagation," Phys. Procedia, vol. 70, pp. 686-689, 2015.

[84] D. K. Stoyko, N. Popplewell, and A. H. Shah, "Detecting and describing a notch in a pipe using singularities," Int. J. Solids Struct., vol. 51, no. 15, pp. 2729-2743, 2014.

[85] V. Y. Senyurek, "Detection of cuts and impact damage at the aircraft wing slat by using Lamb wave method," Measurement, vol. 67, pp. 10-23, 2015. 
[86] S. Ma, Z. Wu, Y. Wang, and K. Liu, "The reflection of guided waves from simple dents in pipes," Ultrasonics, vol. 57, pp. 190-197, 2015.

[87] R. Carandente and P. Cawley, "The effect of complex defect profiles on the reflection of the fundamental torsional mode in pipes," NDT E Int., vol. 46, pp. $41-47,2012$.

[88] T. Kamas, V. Giurgiutiu, and B. Lin, "E/M impedance modeling and experimentation for the piezoelectric wafer active sensor," Smart Mater. Struct., vol. 24, no. 11, p. 115040, 2015.

[89] V. Giurgiutiu and A. Zagrai, "Electro-mechanical impedance method for crack detection in metallic plates," Proc. SPIE - Int. Soc. Opt. Eng., vol. 4335, no. October, pp. 131-142, 2001.

[90] T. Kamas, B. Lin, and V. Giurgiutiu, "Analytical modeling of PWAS in-plane and out-of-plane electromechanical impedance spectroscopy (EMIS),”2013, no. May 2014, p. 869227.

[91] D. Goyal and B. S. Pabla, "Development of non-contact structural health monitoring system for machine tools," J. Appl. Res. Technol., vol. 14, no. 4, pp. 245-258, 2016.

[92] A. Baghalian et al., "Implementation of the Surface Response to Excitation Method for Pipes,” 2017, pp. 261-266.

[93] A. Babaei, A. A. Suratgar, and A. H. Salemi, "Dimension estimation of rectangular cracks using impedance changes of the eddy current probe with a neural network," J. Appl. Res. Technol., vol. 11, no. 3, pp. 397-401, 2013.

[94] D. Donskoy, A. Sutin, and A. Ekimov, "Nonlinear acoustic interaction on contact interfaces and its use for nondestructive testing," NDT E Int., vol. 34, no. 4, pp. 231-238, Jun. 2001. 
[95] K. Kawashima, M. Murase, R. Yamada, M. Matsushima, M. Uematsu, and F. Fujita, "Nonlinear ultrasonic imaging of imperfectly bonded interfaces," Ultrasonics, vol. 44, pp. e1329-e1333, Dec. 2006.

[96] L. Straka, Y. Yagodzinskyy, M. Landa, and H. Hänninen, "Detection of structural damage of aluminum alloy 6082 using elastic wave modulation spectroscopy," NDT E Int., vol. 41, no. 7, pp. 554-563, Oct. 2008.

[97] T. Leutenegger and J. Dual, "Non-destructive testing of tubes using a time reverse numerical simulation (TRNS) method," Ultrasonics, vol. 41, no. 10, pp. 811-822, May 2004.

[98] B. Poddar, A. Kumar, M. Mitra, and P. M. Mujumdar, "Time reversibility of a Lamb wave for damage detection in a metallic plate," Smart Mater. Struct., vol. 20, no. 2, p. 25001, Feb. 2011.

[99] K. E. A. Van Den Abeele, J. Carmeliet, J. A. Ten Cate, and P. A. Johnson, "Nonlinear Elastic Wave Spectroscopy (NEWS) Techniques to Discern Material Damage, Part II: Single-Mode Nonlinear Resonance Acoustic Spectroscopy,” Res. Nondestruct. Eval., vol. 12, no. 1, pp. 17-30, 2000.

[100] K. E.-A. Van Den Abeele, A. Sutin, J. Carmeliet, and P. A. Johnson, "Microdamage diagnostics using nonlinear elastic wave spectroscopy (NEWS)," NDT E Int., vol. 34, no. 4, pp. 239-248, Jun. 2001.

[101] G. Zumpano and M. Meo, "Damage localization using transient non-linear elastic wave spectroscopy on composite structures," vol. 43, pp. 217-230, 2008.

[102] Y.-M. Cheong, M. K. Alam, C. Kim, D. O. Thompson, and D. E. Chimenti, "Nonlinear Parameters For a Diagnosis of Micro-Scale Cracks Using a Nonlinear Resonant Ultrasound Spectroscopy (NRUS)," in AIP Conference Proceedings, 2010, vol. 24, pp. 1439-1444. 
[103] X. Liu, Z. Dao, J. Zhu, W. Qu, X. Gong, and K. Van Den Abeele, "Localization of material defects using nonlinear resonant ultrasound spectroscopy under asymmetric boundary conditions," Phys. Procedia, vol. 3, no. 1, pp. 55-61, 2010.

[104] K. Van Den Abeele, "Multi-mode nonlinear resonance ultrasound spectroscopy for defect imaging: an analytical approach for the one-dimensional case.," J. Acoust. Soc. Am., vol. 122, no. 1, pp. 73-90, 2007.

[105] J. Kober and Z. Prevorovsky, "Theoretical investigation of nonlinear ultrasonic wave modulation spectroscopy at crack interface," NDT E Int., vol. 61, pp. 10-15, 2014.

[106] F. Amerini and M. Meo, "Structural health monitoring of bolted joints using linear and nonlinear acoustic/ultrasound methods," Struct. Heal. Monit., vol. 10, no. 6, pp. 659-672, 2011.

[107] P. Liu, H. Sohn, T. Kundu, and S. Yang, "NDT \& E International Noncontact detection of fatigue cracks by laser nonlinear wave modulation spectroscopy ( LNWMS )," NDT E Int., vol. 66, pp. 106-116, 2014.

[108] H. J. Lim, B. Song, B. Park, and H. Sohn, "Noncontact fatigue crack visualization using nonlinear ultrasonic modulation," NDT E Int., vol. 73, pp. 8-14, 2015.

[109] N. Houhat, T. Boutkedjirt, and V. Tournat, "One-dimensional parametric study of damage detection in a solid material using a Nonlinear Wave Modulation Spectroscopy (NWMS) technique," 2015 4th Int. Conf. Electr. Eng. ICEE 2015, no. October 2016, 2016.

[110] Z. Parsons and W. J. Staszewski, "Nonlinear acoustics with low-profile piezoceramic excitation for crack detection in metallic structures," Smart Mater. Struct., vol. 15, no. 4, pp. 1110-1118, 2006.

[111] T.-C. Poon, "Detection|Heterodyning," Encycl. Mod. Opt., pp. 201-206, 2005. 
[112] D. Havelock, S. Kuwano, and M. Vorländer, Handbook of signal processing in acoustics. 2008.

[113] S. Vijayachitra, Communication Engineering, Sixth Revi. Technical Publications, 2009.

[114] C. Weng, Y. Lin, and W. I. Way, "Radio-Over-Fiber 16-QAM, 100-km Transmission at $5 \mathrm{~Gb} / \mathrm{s}$ Using DSB-SC Transmitter and Remote Heterodyne Detection,” J. Light. Technol., vol. 26, no. 6, pp. 643-653, Mar. 2008.

[115] H. G. Sandalidis, T. A. Tsiftsis, and G. K. Karagiannidis, “Optical Wireless Communications With Heterodyne Detection Over Turbulence Channels With Pointing Errors,” J. Light. Technol., vol. 27, no. 20, pp. 4440-4445, Oct. 2009.

[116] K.-N. Joo, J. D. Ellis, E. S. Buice, J. W. Spronck, and R. H. M. Schmidt, "High resolution heterodyne interferometer without detectable periodic nonlinearity.," Opt. Express, vol. 18, no. 2, pp. 1159-1165, 2010.

[117] T. Pfeifer, L. Gallmann, M. J. Abel, P. M. Nagel, D. M. Neumark, and S. R. Leone, "Heterodyne mixing of laser fields for temporal gating of high-order harmonic generation," Phys. Rev. Lett., vol. 97, no. 16, pp. 1-4, 2006.

[118] N. C. Yoder and D. E. Adams, "Vibro-Acoustic Modulation Utilizing a Swept Probing Signal for Robust Crack Detection," Struct. Heal. Monit., vol. 9, no. 3, pp. 257-267, 2010.

[119] D. M. Joglekar and M. Mitra, “Analysis of flexural wave propagation through beams with a breathing crack using wavelet spectral finite element method," Mech. Syst. Signal Process., vol. 76-77, pp. 576-591, Aug. 2016.

[120] A. Klepka, L. Pieczonka, W. J. Staszewski, and F. Aymerich, "Impact damage detection in laminated composites by non-linear vibro-acoustic wave modulations," Compos. Part B Eng., vol. 65, no. April 2016, pp. 99-108, Oct. 2014. 
[121] R. Watkins and R. Jha, “A modified time reversal method for Lamb wave based diagnostics of composite structures," Mech. Syst. Signal Process., vol. 31, pp. 345-354, 2012.

[122] K. E. Van Den Abeele, A. Sutin, J. Carmeliet, and P. a. Johnson, "Micro-damage diagnostics using nonlinear elastic wave spectroscopy (NEWS)," NDT E Int., vol. 34, no. 4, pp. 239-248, 2001.

[123] M. Meo, U. Polimeno, and G. Zumpano, "Detecting damage in composite material using nonlinear elastic wave spectroscopy methods," Appl. Compos. Mater., vol. 15, no. 3, pp. 115-126, 2008.

[124] Q. He and Y. Lin, "Assessing the severity of fatigue crack using acoustics modulated by hysteretic vibration for a cantilever beam," J. Sound Vib., vol. 370, pp. 306-318, 2016.

[125] G. P. M. Fierro, F. Ciampa, D. Ginzburg, E. Onder, and M. Meo, "Nonlinear ultrasound modelling and validation of fatigue damage," J. Sound Vib., vol. 343, pp. 121-130, 2015.

[126] K. E.-A. Van Den Abeele, J. Carmeliet, J. A. Ten Cate, and P. A. Johnson, "Nonlinear Elastic Wave Spectroscopy (NEWS) Techniques to Discern Material Damage, Part II: Single-Mode Nonlinear Resonance Acoustic Spectroscopy," Res. Nondestruct. Eval., vol. 12, no. 1, pp. 31-42, Jan. 2000.

[127] T. J. Ulrich, M. Remillieux, P.-Y. Le Bas, and C. Payan, "Multimode nonlinear resonant ultrasound spectroscopy (NRUS): From one-dimensional to threedimensional characterization of the hysteretic elastic nonlinearity," J. Acoust. Soc. Am., vol. 138, no. 3, pp. 1886-1886, Sep. 2015.

[128] C. Payan, V. Garnier, J. Moysan, and P. A. Johnson, “Applying nonlinear resonant ultrasound spectroscopy to improving thermal damage assessment in concrete," $J$. Acoust. Soc. Am., vol. 121, no. 4, p. EL125-EL130, Apr. 2007. 
[129] Z. Zhang, H. Xu, Y. Liao, Z. Su, and Y. Xiao, "Vibro-acoustic modulation (VAM)-inspired structural integrity monitoring and its applications to bolted composite joints," Compos. Struct., vol. 176, pp. 505-515, 2017.

[130] J. J. Meyer and D. E. Adams, "Theoretical and experimental evidence for using impact modulation to assess bolted joints," Nonlinear Dyn., vol. 81, no. 1-2, pp. 103-117, 2015.

[131] D. Donskoy, A. Sutin, and A. Ekimov, "Nonlinear Acoustic interaction on contact surfaces and its use for nondestructive testing," NDT E Int., vol. 34, pp. 231-238, 2001.

[132] W.-L. Hu et al., "Dynamic Data Driven Approach for Modeling Human Error," Procedia Comput. Sci., vol. 51, pp. 1643-1654, 2015.

[133] S. M. H. Hosseini, S. Duczek, and U. Gabbert, "Damage localization in plates using mode conversion characteristics of ultrasonic guided waves," J. Nondestruct. Eval., vol. 33, no. 1, pp. 152-165, 2014.

[134] A. Raghavan and C. E. S. Cesnik, "Review of Guided-wave Structural Health Monitoring," Shock Vib. Dig., vol. 39, no. 2, pp. 91-114, Mar. 2007.

[135] G. Park, C. R. Farrar, F. L. Di Scalea, and S. Coccia, "Performance assessment and validation of piezoelectric active-sensors in structural health monitoring," Smart Mater. Struct., vol. 15, no. 6, pp. 1673-1683, 2006.

[136] T. Clarke, P. Cawley, P. D. Wilcox, and A. J. Croxford, "Evaluation of the damage detection capability of a sparse-array guided-wave SHM system applied to a complex structure under varying thermal conditions," IEEE Trans. Ultrason. Ferroelectr. Freq. Control, vol. 56, no. 12, pp. 2666-2678, 2009.

[137] Z. Liu, K. Sun, G. Song, C. He, and B. Wu, "Damage localization in aluminum plate with compact rectangular phased piezoelectric transducer array," Mech. Syst. Signal Process., vol. 70-71, pp. 625-636, 2016. 
[138] N. Quaegebeur, P. Masson, D. Langlois-Demers, and P. Micheau, "Dispersionbased imaging for structural health monitoring using sparse and compact arrays," Smart Mater. Struct., vol. 20, no. 2, p. 25005, 2011.

[139] J. E. Michaels, J. S. Hall, and T. E. Michaels, “Adaptive imaging of damage from changes in guided wave signals recorded from spatially distributed arrays," Proc. SPIE, vol. 7295, no. May 2016, pp. 729515-729515-11, 2009.

[140] J. S. Hall and J. E. Michaels, "Minimum variance ultrasonic imaging applied to an in situ sparse guided wave array," IEEE Trans. Ultrason. Ferroelectr. Freq. Control, vol. 57, no. 10, pp. 2311-2323, 2010.

[141] L. Yu, G. Bottai-Santoni, and V. Giurgiutiu, "Shear lag solution for tuning ultrasonic piezoelectric wafer active sensors with applications to Lamb wave array imaging," Int. J. Eng. Sci., vol. 48, no. 10, pp. 848-861, 2010.

[142] G. Konstantinidis, B. W. Drinkwater, and P. D. Wilcox, "The temperature stability of guided wave structural health monitoring systems," 32nd Annu. Rev. Prog. Quant. Nondestruct. Eval. (AIP Conf. Proc. 820), vol. 25, no. 4, pp. 967-976, 2006.

[143] V. T. Prado, R. T. Higuti, C. Kitano, Ó. Martínez-Graullera, and J. C. Adamowski, "Lamb mode diversity imaging for non-destructive testing of plate-like structures," NDT E Int., vol. 59, pp. 86-95, 2013.

[144] J. S. Hall and J. E. Michaels, "Multipath ultrasonic guided wave imaging in complex structures," Struct. Heal. Monit., vol. 14, no. 4, pp. 345-358, 2015.

[145] A. Muller, B. Robertson-Welsh, P. Gaydecki, M. Gresil, and C. Soutis, "Structural Health Monitoring Using Lamb Wave Reflections and Total Focusing Method for Image Reconstruction,” Appl. Compos. Mater., vol. 24, no. 2, pp. 553-573, Apr. 2017. 
[146] J. Rajagopalan, K. Balasubramaniam, and C. V Krishnamurthy, “A single transmitter multi-receiver (STMR) PZT array for guided ultrasonic wave based structural health monitoring of large isotropic plate structures," Smart Mater. Struct., vol. 15, no. 5, pp. 1190-1196, 2006.

[147] J. Hua, J. Lin, L. Zeng, and F. Gao, "Pulse energy evolution for high-resolution Lamb wave inspection,” Smart Mater. Struct., vol. 24, no. 6, p. 65016, 2015.

[148] C. Holmes, B. W. Drinkwater, and P. D. Wilcox, "Advanced post-processing for scanned ultrasonic arrays: Application to defect detection and classification in nondestructive evaluation," Ultrasonics, vol. 48, no. 6-7, pp. 636-642, 2008.

[149] J. Zhang, B. W. Drinkwater, P. D. Wilcox, and A. J. Hunter, "Defect detection using ultrasonic arrays: The multi-mode total focusing method," NDT E Int., vol. 43, no. 2, pp. 123-133, 2010.

[150] P. D. Wilcox, "Omni-directional guided wave transducer arrays for the rapid inspection of large areas of plate structures," IEEE Trans. Ultrason. Ferroelectr. Freq. Control, vol. 50, no. 6, pp. 699-709, 2003.

[151] W. a. K. Deutsch, a. Cheng, and J. D. Achenbach, "Self-Focusing of Rayleigh Waves and Lamb Waves with a Linear Phased Array," Res. Nondestruct. Eval., vol. 9, no. 2, pp. 81-95, 1997.

[152] E. Moulin, N. Bourasseau, J. Assaad, and C. Delebarre, "Lamb-wave beamsteering for integrated health monitoring applications," in Nondestructive Evaluation and Health Monitoring of Aerospace Materials and Composites Ii, 2003, vol. 5046, no. August, p. 124.

[153] A. C. Clay, S. C. Wooh, L. Azar, and J. Y. Wang, "Experimental study of phased array beam steering characteristics," J. Nondestruct. Eval., vol. 18, no. 2, pp. 59$71,1999$. 
[154] V. Giurgiutiu and J. Bao, "Embedded-ultrasonics Structural Radar for In Situ Structural Health Monitoring of Thin-wall Structures," Struct. Heal. Monit., vol. 3, no. 2, pp. 121-140, 2004.

[155] S. J. Orfanidis, Electromagnetic Waves. 2001.

[156] L. Yu and V. Giurgiutiu, "In situ 2-D piezoelectric wafer active sensors arrays for guided wave damage detection," Ultrasonics, vol. 48, no. 2, pp. 117-134, 2008.

[157] P. D. Wilcox, "A rapid signal processing technique to remove the effect of dispersion from guided wave signals," IEEE Trans. Ultrason. Ferroelectr. Freq. Control, vol. 50, no. 4, pp. 419-427, 2003. 
VITA

\section{AMIN BAGHALIAN}

\section{EDUCATION}

$2002-2007$

B.Sc., Mechanical Engineering- Manufacturing \& Production University of Mazandaran (the later Babol Noshirvani University of Technology)

Babol, Iran

$2008-2010$

M.Sc., Mechanical Engineering- Manufacturing \& Production University of Mazandaran (the later Babol Noshirvani University of Technology)

Babol, Iran

$2013-2017$

$\mathrm{PhD}$ Candidate, Mechanical Engineering Florida International University Miami, Florida, USA

- Dissertation Year Fellowship (DYF), FIU, Fall 2017.

- GSAW Provost Awards for best oral presentation, FIU, Spring 2017.

- Doctoral Evidence Acquisition (DEA) Fellowship, FIU, Spring 2017.

- GSAW Provost Awards for best poster presentation, FIU, Spring 2016.

\section{JOURNAL PUBLICATIONS \& PROCEEDINGS}

[1] A. Baghalian, S. Tashakori, V. Y. Senyurek, D. Mcdaniel, H. Fekrmandi, and I. N. Tansel, "Non-Contact Quantification of Longitudinal and Circumferential Defects in Pipes using the Surface Response to Excitation (SuRE) Method," Int. J. Progn. Heal. Manag., vol. 8, no. 2, p. 8, 2017.

[2] S. Tashakori, A. Baghalian, M. Unal, H. Fekrmandi, V. Y. Şenyürek, D. McDaniel, and I. N. Tansel, "Contact and Non-Contact Approaches in Load Monitoring Applications Using Surface Response to Excitation Method," Measurement, vol. 89, pp. 197-203, 2016. 
[3] H. Fekrmandi, M. Unal, A. Baghalian, S. Tashakori, K. Oyola, A. Alsenawi, and I. N. Tansel, "A Non-Contact Method for Part-based Process Performance Monitoring in End Milling Operations," Int. J. Adv. Manuf. Technol., 2015.

[4] V. Y. Senyurek*, A. Baghalian*, S. Tashakori, D. McDaniel, and I. N. Tansel, "Localization of Multiple Defects Using the Compact Phased Array (CPA) Method," J. Sound Vib., vol. 413, pp. 383-394, 2018. * These authors contributed equally to this work

[5] A. Baghalian, S. Tashakori, J. R. Soto, V. Y. Senyurek, I. N. Tansel, and B. Uragun, "Internal defect detection in hollow cylindrical structures using the Surface Response to Excitation (SuRE) Method," in 2017 8th International Conference on Recent Advances in Space Technologies (RAST), 2017, pp. 523-527.

[6] A. Baghalian, S. Tahakori, H. Fekrmandi, M. Unal, V. Y. Senyurek, D. McDaniel, I. N. Tansel, "Implementation of the Surface Response to Excitation Method for Pipes,” Springer International Publishing, 2017, pp. 261-266.

[7] A. Baghalian, S. Tashakori, V. Y. Senyurek, M.Unal, I. N. Tansel, "Novel Approaches for Loose Bolt Detection with and without Sensors Using Heterodyning Effect," The 11th International Workshop on Structural Health Monitoring, September 12-14, 2017, Stanford, California, USA.

[8] I. N. Tansel, V. Y. Şenyürek, A. Baghalian, M. Unal, S. Tahakori "Loose Bolt Detection Using Smart Washers, " FCRAR-2016, Miami, FL, USA.

[9] I. N. Tansel, V. Y. Şenyürek, M. Unal, A. Baghalian, S. Tahakori “Implementation of Heterodyne Effect in Structural Health Monitoring (SHM) Systems, " FCRAR2016, Miami, FL, USA.

[10] I. N. Tansel, V. Y. Şenyürek, M. Unal, A.Baghalian, S. Tahakori "Loose Bolt Detection Using a Sensorless SHM System, ” FCRAR-2016, Miami, FL, USA. 